\title{
Development of the Raman spectroscopic technique for in vivo applications in the eye
}

Citation for published version (APA):

Erckens, R. J. (2001). Development of the Raman spectroscopic technique for in vivo applications in the eye. [Doctoral Thesis, Maastricht University]. Universiteit Maastricht. https://doi.org/10.26481/dis.20010705re

Document status and date:

Published: 01/01/2001

DOI:

10.26481/dis.20010705re

Document Version:

Publisher's PDF, also known as Version of record

\section{Please check the document version of this publication:}

- A submitted manuscript is the version of the article upon submission and before peer-review. There can be important differences between the submitted version and the official published version of record.

People interested in the research are advised to contact the author for the final version of the publication, or visit the DOI to the publisher's website.

- The final author version and the galley proof are versions of the publication after peer review.

- The final published version features the final layout of the paper including the volume, issue and page numbers.

Link to publication

\footnotetext{
General rights rights.

- You may freely distribute the URL identifying the publication in the public portal. please follow below link for the End User Agreement:

www.umlib.nl/taverne-license

Take down policy

If you believe that this document breaches copyright please contact us at:

repository@maastrichtuniversity.nl

providing details and we will investigate your claim.
}

Copyright and moral rights for the publications made accessible in the public portal are retained by the authors and/or other copyright owners and it is a condition of accessing publications that users recognise and abide by the legal requirements associated with these

- Users may download and print one copy of any publication from the public portal for the purpose of private study or research.

- You may not further distribute the material or use it for any profit-making activity or commercial gain

If the publication is distributed under the terms of Article $25 \mathrm{fa}$ of the Dutch Copyright Act, indicated by the "Taverne" license above, 


\section{Development of the Raman spectroscopic technique for in vivo applications in the eye.}

Roel J. Erckens 


\section{R.J. Erckens}

Development of the Raman spectroscopic technique for in vivo applications in the eye. ISBN 90-5681-109-6

Vormgeving en print: Unigraphic, Universiteit Maastricht 


\section{Development of the Raman spectroscopic technique for in vivo applications in the eye.}

\section{PROEFSCHRIFT}

ter verkrijging van de graad van doctor aan de Universiteit Maastricht op gezag van de Rector Magnificus

Prof. dr. A. Nieuwenhuijzen Kruseman volgens het besluit van het College van Decanen

in het openbaar te verdedigen

op donderdag 5 juli 2001 om 16.00 uur

door

\section{Roel Johan Erckens}

Geboren op 4 februari 1959 te Meerssen 


\section{Promotores:}

Prof. dr. F. Hendrikse

Prof. dr. W. March (University of Texas Medical Branch, Galveston TX, USA)

Co-promotor:

Dr. J. Wicksted (Oklahoma State University, Stillwater OK, USA)

Dr. F. Jongsma

\section{Beoordelingscommissie:}

Prof. dr. H. Struijker Boudier (voorzitter)

Prof. dr. L. Koole

Prof. dr. ir. J. Janssen

Prof. dr. ir. M. van Gemert (Universiteit van Amsterdam)

Dr. ir. H. Sterenborg (Erasmus Universiteit Rotterdam) 
'Nothing in life is to be feared; it is only to be understood'. Marie Curie.

(In: Analytical Applications of Spectroscopy II, A. Davies and C. Creaser, 1991 The Royal Society of Chemistry, Cambridge Great Britain)

For my parents 


\section{Contents}

1 Introduction and aim of the study

2 Raman spectroscopy in ophthalmology: From experimental tool to applications in vivo. (In press in Lasers in Medical Science).

3 Raman spectroscopy studies of metabolic concentrations in aqueous solutions and aqueous humor specimens. (Applied Spectroscopy 1995).

4 Raman spectroscopy for noninvasive characterization of ocular tissue: Potential for detection of biological molecules. (J Raman Spectr 1997).

5 Confocal Raman spectroscopy system for noncontact scanning of ocular tissues: An in vitro study. (Opt Eng 1997).

6 Drug induced corneal hydration changes monitored in vivo by non-invasive confocal Raman spectroscopy. (In press in J Raman Spectr).

7 Noninvasive in vivo assessment of rabbit lens hydration using confocal Raman spectroscopy.

8 Raman spectroscopy: noninvasive determination of silicone oil in the eye. Potential applications for intraocular determination of biomaterials. (Submitted).

9 Noninvasive Raman spectroscopic identification of the intraocular lens material in the living human eye. (In press in J Cat Refr Surg).

Summary and conclusions.

Samenvatting en conclusies.

Dankwoord.

Curriculum Vitae

Publications 


\section{Introduction}

When the eye seems to be threatened by a disease or a traumatic event, an accurate examination is required to detect ocular changes in order to, if necessary, initiate treatment to prevent severe visual disturbances or even blindness. Due to the optical properties of the eye, a variety of noninvasive optical techniques can be applied for diagnostic as well as for treatment purposes. The eye is the only organ where blood vessels, blood flow and nerve tissue can be visualized non invasively, which enables study of the eye structures with different optical devices. For instance the local shape, thickness, color, integrity and transparency of the tissue can be examined with the slit lamp biomicroscope as noted by A. Gullstrand, the Nobel laureate in $1911^{(1)}$. In Figure $1 \mathrm{~A}$ the anatomical structures of the eye are shown. In Figure 1B the optical setup of the biomicroscope is shown. The focusing and collecting optics are independently adjustable, which enables the examiner to change the viewing angle or the viewing depth.

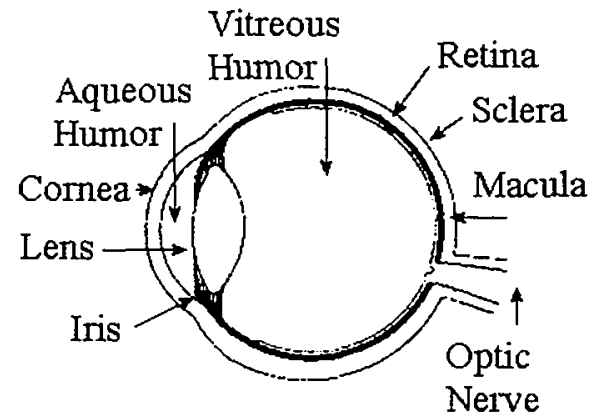

Fig. IA.

Anatomical structures of the eye.

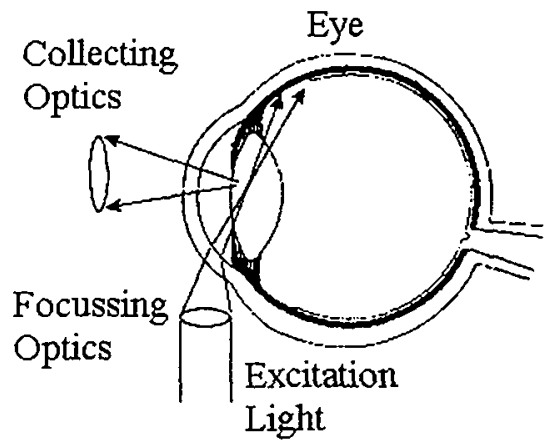

Fig. $1 B$.

Optical setup of the biomicroscope.

A laser(2) may be the ideal light source for treatment and diagnostic purposes in ophthalmology. This is because the laser provides monochromatic light (i.e. light of one color) to match the specific absorption coefficient of the target tissue and to match the transmission coefficient of the tissue to be spared. Furthermore, sufficient energy either pulsed or continuous wave is available, combined with a small (adjustable) spot size to deliver the energy to the exact location in the tissue that needs to be treated. In Figure 2 an example of a laser line at $632.8 \mathrm{~nm}$ is shown.

In ophthalmology, lasers ${ }^{(3,4)}$ have evolved into indispensable therapeutic tools. Besides coagulation, specific lasers are able to disrupt atomic bonds, a property used in cornearefractive surgery for treatment of refractive errors, or to create a small shockwave in the treatment of a posterior capsule opafication after cataract surgery. The laser can be used in glaucoma to create a peripheral iridectomy to treat or prevent an angle closure glaucoma, or to enhance the outflow of aqueous humor by means of a laser trabeculoplasty. In the treatment of dia- 


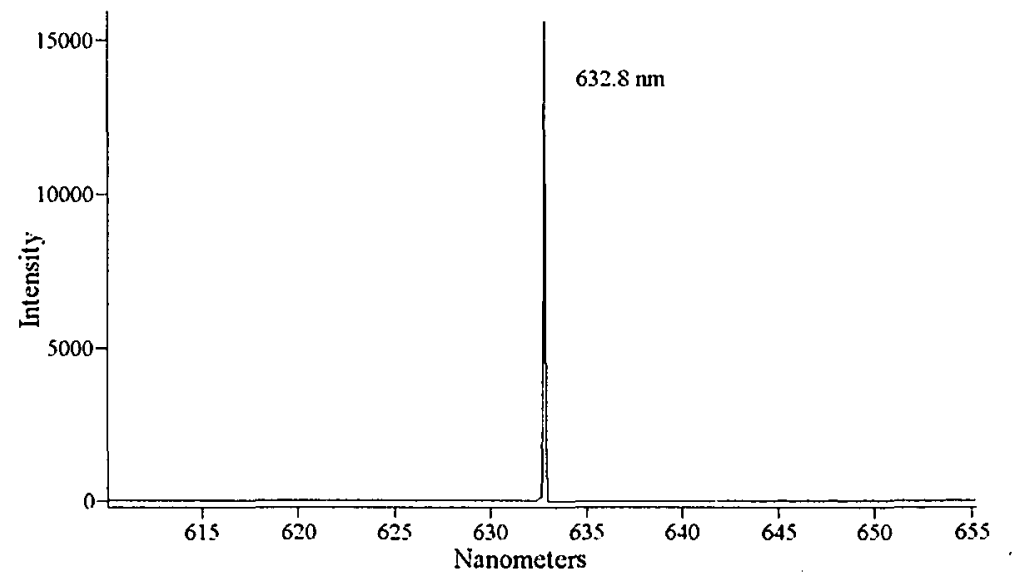

Fig. 2. HeNe laser line.

betic retinopathy or in venous occlusive diseases the areas of nonperfusion or ischemia injury can be obliterated using the focused laser light in order to prevent neovascularization or to decrease edema.

Several diagnostic techniques using light are available to obtain information with numerical and repeatable values or images of the different eye tissues. These include specular microscopy(5) and confocal microscopy(6) to study the cornea cell layers, cornea topography(7) to measure the curvature of the cornea, fluorescein angiography(8) to study the retinal blood flow and to detect alterations in fluid dynamics. These and other techniques like fluorometry, fluorophotometry and Scheimpflug photography are discussed in detail by Masters(9). The laser flare meter ${ }^{(10)}$ gives information on the amount of total proteins in the anterior chamber, Optical Coherence Tomography(11,12) enables to visualize and measure different eye structures, the Laser Scanning Ophthalmoscope ${ }^{(13.14)}$ provides information on retinal blood flow or optic nerve alterations, the retinal thickness analyzer provides information on retinal and macular thickness(15). The use of the laser as a diagnostic tool is limited due to the strict safety requirements in order to prevent unwanted damage to the most sensitive layer of all eye tissues, namely the retina. The American National Standard for the Safe Use of Lasers (16) gives maximum permissible exposure (MPE) to prevent laser light damage in tissues, including the retina. Utilizing these safety standards, Bauer(17) estimated that the MPE at a wavelength of $514.5 \mathrm{~nm}$ should be below $3 \mathrm{~mJ}$ if the retinal area is about $2.7 \mathrm{~cm}^{2}$ with an exposure time equal or longer than 1 second.

However, to obtain in vivo information qualitatively as well as quantitatively of the normal physiologic processes or the pathologic state without interfering with the integrity of eye tissue, poses a difficult but intriguing challenge. Currently, qualitative as well as quantitative information on hydration status or chemical composition (e.g. healing processes in the cornea, changes in the aqueous humor contents, maintaining optical transparency, cataract formation) of ocular tissues, or detection of intra ocular substances (e.g. disturbed healing processes of the cornea, ocular inflammation, changes to eye tissue due to systemic diseases) needs to be addressed with histochemical examinations of specimens obtained by 
paracenthesis or by biopsy. These invasive techniques disturb the microenvironment of the eye and carry the risk of infection or contagious diseases to spread.

For diagnostic purposes, spectroscopy can be used for molecular or tissue determination. Spectroscopy is a powerful technology that could play a major role as a tool of nondestructive studying of tissue in situ.

\section{Spectroscopy}

Spectroscopy is the science that studies the interaction of light with any matter. This is not limited to the visible light, but includes electromagnetic radiation with shorter or longer wavelengths than the visible spectrum. In 1666 Newton(18) observed that when white light is passed through a glass prism, the colors of the rainbow could be seen. The rainbow itself is a natural spectrometer in the sky where the sun is the light source and the water molecules in the atmosphere act as the dispersive elements and our eyes are the detectors. In the early nineteenth century(19), Fraunhofer showed that the spectrum of the sun contains a number of dark lines due to absorption of photons in gas. In the later nineteenth century, Kirchhoff developed the spectroscope and discovered, together with Bunsen, the spectral bands specific for atomic species. They discovered two new elements, cesium and rubidium, by observing the specific spectral lines. Hence the discovery of the dispersion of light initiated among others the studying of emitted radiant energy arising from molecules in our everyday life to distant objects in the universe.

Different types of optical spectroscopic methods include fluorescence, chemiluminescence, phosphorescence, absorption, emission, and scattering. The radiant energy from the sample is usually weak and for a prolonged period of time the use of spectroscopy was limited. However, the introduction of the laser and the development of quantum efficient detectors proved to be a major step forward in applying the spectroscopic techniques. Currently, the more common used types ${ }^{(20)}$ of spectroscopies are absorption, fluorescence, and scattering (Raman) spectroscopy. The limitation to use absorption spectroscopy in biological tissue or in other water environments, is the high absorption coefficient of water for infrared photons, because the measurement of wavelength and intensity of the absorption of a photon in the sample is usually obtained with infrared radiation. In Figure 3, a Fourier transform infrared (FTIR) absorption spectrum is shown of water using a Nernst Glower (broad infrared source). The upper dashed line is pure water, the other lines are water where $\mathrm{HCl}$ was added to change the $\mathrm{pH}$. In this figure the ordinate is linear in transmittance and the (1) in the upper left corner means $100 \%$ transmission. So the ordinate shows the ratio of the intensity of the transmitted-light through the sample to the incident light on the sample. (The absorption coefficient is obtained by taking the logarithm of this ratio and divide it by minus the thickness of the sample). The abscissa in this Figure is linear in wavenumbers in units of reciprocal centimeters, while also the wavelength in $\mu \mathrm{m}$ is given. Note that the horizontal scale changes at $2000 \mathrm{~cm}^{-1}$ for convenience purposes. This is due to the fact that most qualitative infrared detail appears at wavenumbers smaller than $2000 \mathrm{~cm}^{-1}$.

In fluorescence, in which atoms or molecules are excited to a higher energy level followed by a decay to lower levels thereby emitting light, the activity is dependent on a fluorophore 
and it is difficult to yield biochemical information of a fluorescent sample due to broader spectral features and spectral overlap.

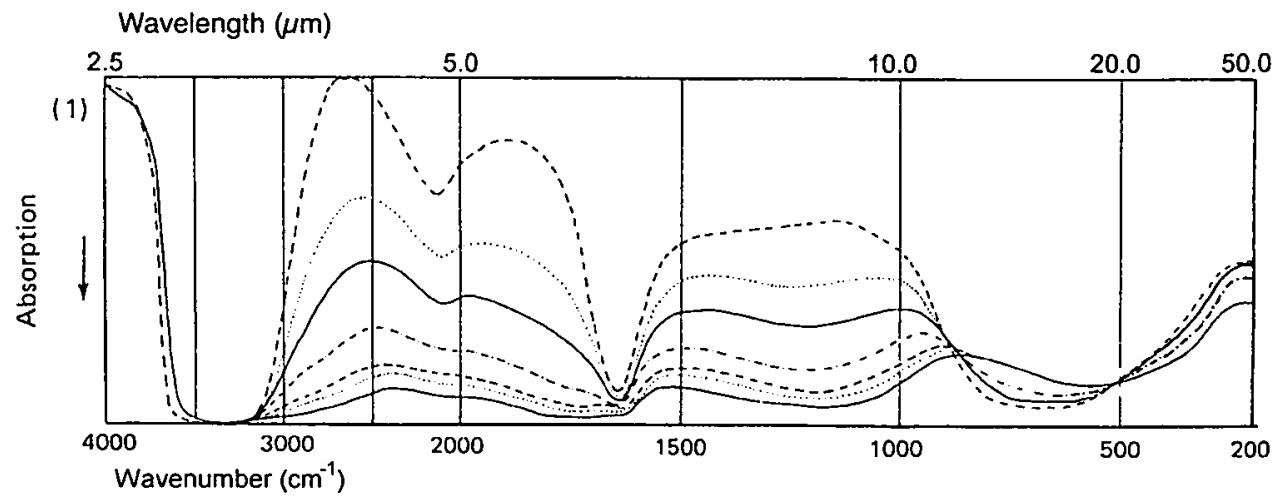

Fig. 3. FT-IR absorption spectrum of water.

\section{Raman spectroscopy}

Raman spectroscopy is an accurate and precise technique that provides qualitative and quantitative information about the molecular composition of screened samples. With this technique it is possible to obtain the required information in vivo without interfering with the integrity of the eye tissue.

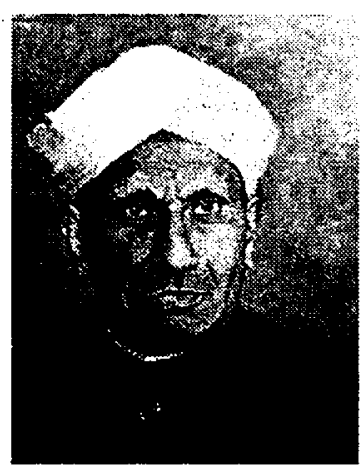

Fig. 4. Sir CV Raman

In 1930 the Indian scientist C.V. Raman received the Nobel prize for his discovery in 1928 of a new phenomenon in light scattering(21) from materials (Fig. 4). He discovered that illumination of a material with monochromatic light not only gives Rayleigh scattering and fluorescence, if a fluorescent molecule is present, but that there was another, more feeble scat- 
tering event. Rayleigh scattering occurs when a photon collides with a molecule and there is no exchange of energy between the photon and the molecule. The wavelength of the photon is the same before and after the collision (an elastic scattering). Fluorescence, named after the mineral fluorite by G. Stokes, occurs if a fluorescent molecule absorbs light which is followed of a delayed emission of light at a longer wavelength. In addition, some photons collide with the molecule and cause an exchange of energy (an inelastic scattering event or the Raman effect). Consequently, the wavelength of the photon before the collision differs from the wavelength after the collision. Generally, the scattered photon has less energy (longer wavelength) than the impinging photon. We call this a 'Stokes' shift. This is named after G. Stokes who's general law states that fluorescent radiation is not of higher frequency than the incident light, and since the Stokes shift in the Raman phenomena follows this law it is also used in conjunction with Raman. At room temperature the Stokes shift will normally be the most noticeable shift. However, when the temperature is raised, much more molecules will go into states of higher vibrational energy, which can be transferred to the colliding photon. In that case the scattered photon has gained energy and consequently has a higher frequency (shorter wavelength). As this is not according to Stokes general law, this shift is called the 'anti-Stokes' shift. Figure 5 shows a quantum mechanic presentation of the possible photon/matter interactions. Figure 6 shows a Stokes/anti-Stokes spectrum of Carbon Tetrachloride $\left(\mathrm{CCl}_{4}\right)$ at room temperature. Experimental settings: HeNe laser at 632.8 $\mathrm{nm}, 30 \mathrm{~mW}, 1$ second. The $\mathrm{X}$-axis of the figure shows the Raman shift in wavenumbers plotted with respect to the incident (i.e. exciting) laser light which is denominated $0 \mathrm{~cm}^{-1}$. The Raman scattered light can be shifted from the incident light as much as 4000 wavenumbers.

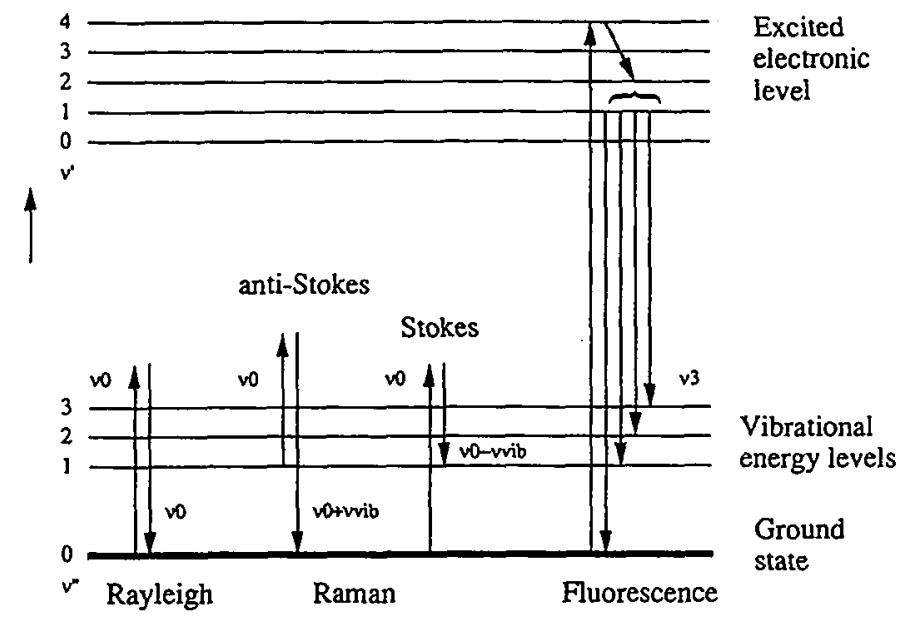

Fig. 5. Quantum mechanic model of possible photon-matter interaction.

During the scattering process the total energy is conserved. This implies that the energy change of the photon must be equal to the energy change within the molecule. So by measuring the energy lost or gained by the photon we can detect transitions between molecular 


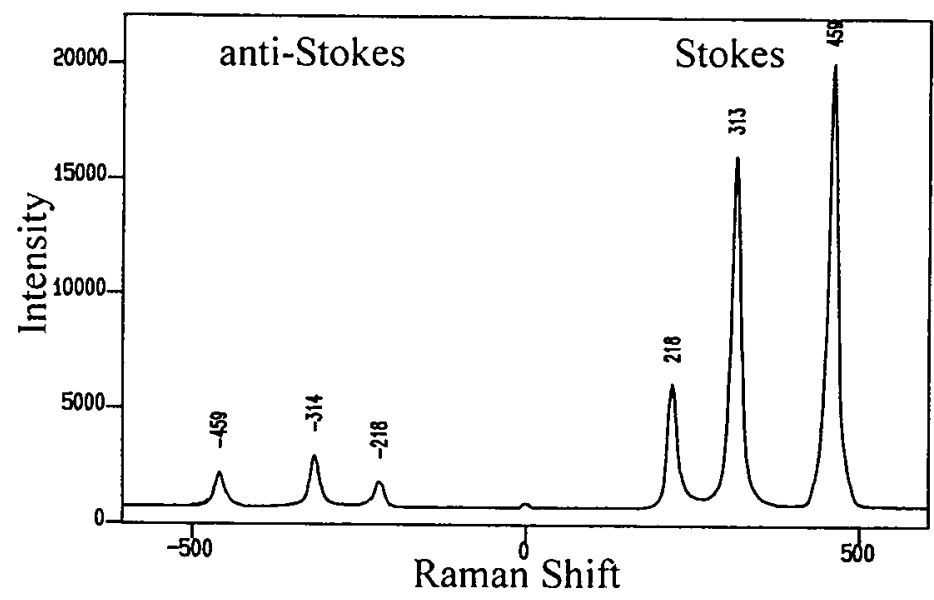

Fig. 6. Raman spectrum of $\mathrm{CCl}_{4}$.

energy levels, hence the Raman spectrum reveals a vibrational spectrum of a molecule. In order for the vibration to inelastically scatter radiation, a change in polarizability (i.e. the ease of which an electron cloud can be distorted by an external electric field and depends on how tightly the electrons are bound to the nuclei) during the vibration needs to take place. The vibrational motions of molecules are sensitive to chemical changes, indicating that the vibrational spectrum can be used to monitor molecular chemistry. The frequency of a Raman shift is independent of the exciting laser wavelength, however, the Raman intensity is inversely proportional to the fourth power of the incident light wavelength $\left(I=\propto / \lambda^{4}\right)$

More characteristic features of Raman spectroscopy are:

- The Raman effect is weak, about $10^{6}$ to $10^{8}$ smaller than Rayleigh scattering.

- The Raman effect is an instantaneous light scattering effect (Timescale about $10^{-15} \mathrm{~s}$ ). The sample scatters instantaneously a small amount of light that differs in wavelength of the incoming light. Fluorescence is an effect that occurs with delay at a time scale of $10^{-9}$ to $10^{-12}$ seconds and is about $10^{6-10^{8}}$ times stronger in intensity than Raman scattering.

- Raman can identify the chemical composition of a sample and can extract the percentage weights in the samples. The intensity of the Raman scattering is directly proportional to the concentration of the substance under investigation.

- Raman is well suited for investigation in aqueous environment since the water Raman activity is relatively weak. There is almost no solvent interaction in the range between $200-2000 \mathrm{~cm}^{-1}$, except at $1640 \mathrm{~cm}^{-1}$ where a bending mode of water is located. This interaction is not interfering much in most cases with the Raman spectrum of the biological material. For in vitro studies glass or quartz cuvettes can be used to hold the samples since these materials have a weak Raman signature.

- The volume of the sample can be small.

- Raman spectra can be obtained under different experimental conditions. 
In Raman experiments a high monochromatic photon flux on the sample is necessary to generate detectable Raman signals. In the early days after the discovery of the Raman effect obtaining Raman spectra was a cumbersome procedure requiring large samples and very long collecting times $(24 \mathrm{~h})$. The reason for this was that there existed no adequate light source. In fact, the experiment performed by Raman used filtered sunlight to obtain the data. An improvement was the introduction of the Toronto arc source in 1952. But it was not until the sixties that the significant leap forward came with the introduction of the laser. Now there was a light source available that was highly monochromatic, generated high power densities, had a long coherence length and a small beam diameter. For this reason the laser is the superior light source. The scattered light is collected and directed into a spectrometer. The function of the spectrometer is to separate spatially the scattered light on basis of frequency. In the spectrometer an image is formed of the Raman spectrum consisting of faint lines. This image at the exit port of the spectrometer is projected on the means of detection: A photographic plate; a photomultiplier tube; or a charge coupled device (CCD) detector. The advantage of a photographic plate and the $\mathrm{CCD}$ detector is that the spectrum is recorded simultaneously, while a photomultiplier tube requires a scanning monochromator, which sequentially records the spectra. Since the CCD detector has a high quantum efficiency, even the faint Raman spectra can be recorded in milliseconds. This is essential while performing in vivo studies.

\section{State of the art at the beginning of this thesis}

Research on ocular tissue with Raman spectroscopy was mainly focused on studies on retina and lens research in vitro. The first in vivo study on lens tissue in a rabbit was performed by $\mathrm{Yu}$ in 1982(22). The main problem was the high photon flux on the tissue or consequently long exposure times necessary to obtain useful Raman spectra.

Chapter 2 of this thesis gives a review of research performed with Raman spectroscopy on ocular tissues.

\section{Aim of the thesis}

The objective of the research was to develop a safe clinical diagnostic tool to obtain information on the molecular composition of the eye.

To achieve this goal, an optical spectroscopic technique based on Raman scattering was applied. The advantage of Raman spectroscopy is the ability to determine the molecular structure in matter, not only qualitative but also quantitative. However, the Raman effect is about $10^{6}$ to $10^{8}$ weaker than the other scattered light (i.e. Rayleigh and/or fluorescence), which implies that the collection of the Raman scattered photons needs to be extremely efficient. To avoid light damage to retina tissue, the amount of photons needed to generate a Raman spectrum, has to be limited. 


\section{Outline}

Initially, the sample chamber of the spectrometer was utilized which allowed studying the specimens either in a capillary tube, cuvette or mounted on a holder. This is described in chapter 3.

Next we studied samples with a commercially available Raman excitation and detection probe. This enabled us to examine the potentials and drawbacks. However, it was not possible to obtain in vivo data due to high laser power settings and a high loss of Raman shifted photons. This is described in chapter 4.

The trade off is between the use of a minimum amount of exciting photons combined with a maximum amount of collected Raman scattered photons. In order to use a minimum amount of photons, the excitation and collection of the photons needs to be optimized. For this reason the confocal approach was used combined with a high light gathering power of the probe.

In a confocal setup, both lateral and axial resolution are enhanced due to a narrowing of the point spread function(23). In a sample, a single point is illuminated by a point light source and instantly projected on a point detector. Marvin Minsky developed the first confocal microscope in 1955(24). In Minsky's design, the condenser focused the light source within a small area of tissue while simultaneously focusing the microscope objective on the same area. So the condenser and objective lens have the same focal point, hence the term confocal. This approach has several advantages: Out of the focal plane the power density decreases dramatically, while confocal also limits fluorescence arising from adjacent tissue to reach the detector, and the sampling depth can be adjusted. For this, we developed a confocal large aperture probe design in a backscatter configuration that allows investigating the different eye structures up to the corpus vitreous (Fig. 7). This is described in chapter 5. This probe was used in our experiments to perform in vivo measurements in the rabbit cornea in chapter 6, and the rabbit lens in chapter 7. Ultimately, two applications for usage in patients could be developed. In the anterior chamber of a patient, silicone oil could be detected as described in chapter 8 . The material of an implanted intraocular lens (IOL) could be determined in patients as described in chapter 9 . Figure 8 shows the setup as used in chapters 8 and 9.

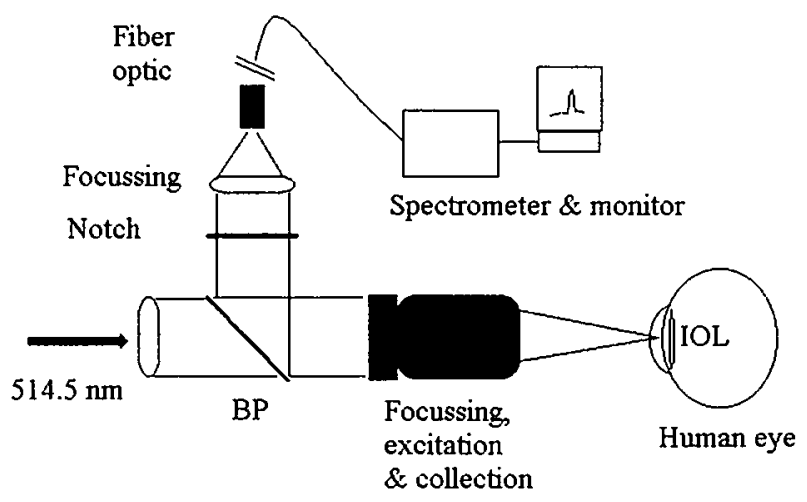

Fig. 7. Setup of the confocal configuration. 


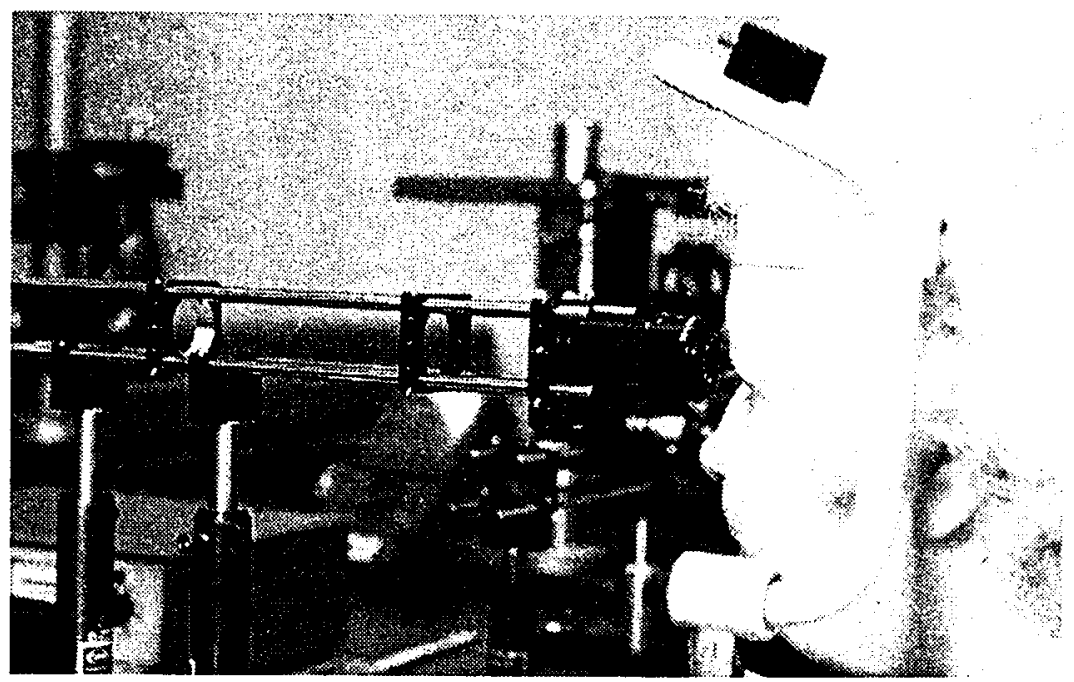

Fig. 8. The confocal probe while performing a measurement on a patient.

\section{References}

1. Duane's Clinical Ophthalmology. Lippincott-Raven Publishers, Hagerstown MD 1997.

2. Maiman T. Stimulated optical radiation in ruby. Nature 1960:187:493.

3. March WM. Opthalmic lasers: Current clinical uses. Slack, Thorofare NJ 1984.

4. Weingeist TA, Sneed SR. Laser surgery in ophthaimology. Appleton \& Lange, Norwalk CT 1992.

5. Sugar A. Clinical specular microscopy. Surv. Ophthalmol 1979;24:21-32.

6. Pawley JB. Handbook of biological confocal microscopy. Plenum Press New York NY 1990.

7. Jongsma FH, de Brabander J, Hendrikse F. Review and classification of corneal topographers. Lasers Med Sci 1999; 14:2-19. Review.

8. Novotny HR. Alvis DL. A method of photographing fluorescence in circulating blood of the human retina. Circulation $1961 ; 24: 82$.

9. Masters B. Noninvasive diagnostic techniques in Ophthalmology. New York: Springer-Verlag, 1990.

10. Sawa M. Clinical application of laser flare-cell meter. Jap. J. Ophthal. 1990;34:346-363.

11. Huang D, Swanson E, Lin CP et al. Optical coherence tomography. Science 1991:254:1178-81.

12. Hoerauf $\mathrm{H}$, Wirbelauer $\mathrm{C}$, Scholz $\mathrm{C}$. et al. Slit-lamp-adapted optical coherence tomography of the anterior segment. Graefe's Arch Clin Exp Ophthalmol 2000; 238:8-18.

13. Webb R. Scanning Laser Ophthalmoscope. IEEE Transact Biomed Eng 1981:28:488-492.

14. Weinreb RN, Dreher AW, Bille J. Quantitative assessment of the optic nerve head with the laser tomographic scanner. Int J Ophthalmol 1989:13:125.

15. Landau D, Schneidman E, Jacobovitz T, Rozenman Y. Quantitative in vivo recinal thickness measurements in healthy subjects. Ophthalmology 1997;104:639-642.

16. ANSI. (1993). American National Standards Institute. Safe use of lasers. In: ANSI standard Z 136.1. Laser Institute of America, Orlando U.S.A. 1993.

17. Bauer NJ. Potential applications of confocal Raman spectroscopy in ophthalmology. Thesis University of Maastricht, the Netherlands 1999. 
18. Sir Isaac Newion. Opticks. Dover publications New York NY 1979. based on the fourth edition London 1730.

19. Purrington RD. Physics in the Nineteenth Century. Rutgers University Press New Brunswick NJ 1997.

20. Skoog D, Leary J. Principles of instrumental analysis. Saunders College Publishing/ Harcour Brace College Publishers Orlando FL 1992.

21. Raman C, Kirishnan K. A new type of secondary radiation. Nature. 1928:121:105.

22. Yu NT, Kuck J, Askren C. Laser Raman Spectroscopy of the Lens in situ, measured in an anesthecized rabbit. Curr Eye Res 1982;1;10:615-618.

23. Wilson T and Sheppard C. Theory and practice of scanning optical microscopy. London: Academic Press, 1984.

24. Minsky M. Memoir on inventing the confocal scanning microscope. Scanning 1988:10:128-38. 


\title{
Chapter 2
}

\section{Raman spectroscopy in ophthalmology: From experimental tool to applications in vivo.}

In press in Lasers in Medical Science

\author{
R.J. Erckens', F.H.M. Jongsma' ${ }^{1}$, J.P. Wicksted ${ }^{2}$, F. Hendrikse', W.F. March?', \\ M. Motamedi3.
}

1 Department of Ophthalmology, Academic Hospital Maastricht, Maastricht, The Netherlands.

2 Department of Physics. Center for Sensors \& Sensor Technologies. Oklahoma State University. Stillwater. Oklahoma 74078-3072, U.S.A.

3 Laser \& Spectroscopy Program of the Center for Biomedical Engineering and the Department of Ophthalmology and Visual Sciences, University of Texas Medical Branch Galveston. Galveston. Texas 77555-0456. U.S.A. 


\begin{abstract}
Raman spectroscopy is a qualitative and quantitative optical technique for determining the molecular composition of matter. Improvements in spectroscopic instruments, especially the modality to detect low light level signals extended the Raman technique to biomedical applications, even in delicate structures like the eye. The purpose of this paper was to make an inventory of performed applications of Raman spectroscopy in biomedical science and especially in ophthalmology. A literature search was done using Medline, Current Contents, a patent server on the Internet, and references found in articles and patents. This search revealed a variety of Raman techniques and applications in biomedical research, and an increasing flow of articles starting in the late 1970s on Raman spectroscopy in ophthalmology. This increase in literature about Raman spectroscopy in ophthalmology feeds the expectation that this valuable technique will be introduced in the future in clinical practice.
\end{abstract}

Key words: Confocal microscopy, eye tissue, ophthalmology, optical sectioning, Raman spectroscopy. 


\section{Introduction}

The eyes from higher developed species including the human are made of optical transparent structures in order to bring the environmental light on the light detector cells of the retinal tissue(1). Retinal cells convert the light into an electric signal, which is further transported to the brain tissue by means of the optic nerve. As the eye is made from different structures (Fig. 1), each structure plays a part in developing specific altered states due to aging, local or systemic diseases, or trauma-related events. A few well-known examples(1) starting from the anterior part include: Cornea opacities may occur due to inherited or internal diseases, trauma or a high eye pressure; the biochemical constitution of the aqueous humor may alter due to disease like diabetes, or exhibit the Tyndall effect (accumulation of proteins) in case of uveitis; the lens develops a cataract due to aging, pharmacological substances (e.g. corticosteroids) and trauma; the vitreous humor may be altered by systemic diseases like diabetes; and finally the retina tissue may show alterations due to age (age-related macular degeneration), systemic disease like diabetes or hypertension (diabetic or hypertension retinopathy) and trauma. The retina and vitreous humor may exhibit a proliferative vitreoretinopathy after a retinal tear or a retinal detachment, particularly after surgery to correct the tear or detachment. In all of the above-mentioned eye tissues, an infection either by bacteria or viruses may alter the basic composition of the tissue involved.

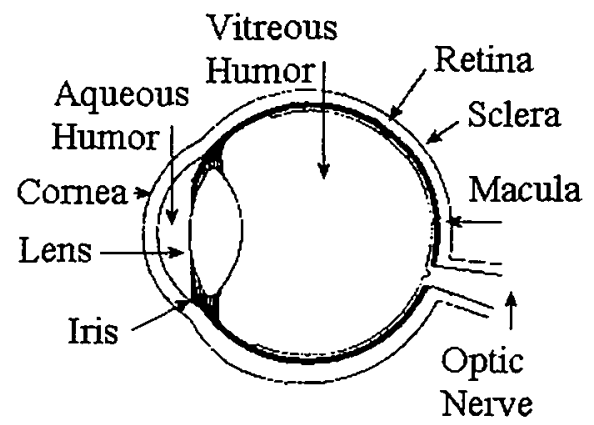

Fig. 1. Relevant anatomical regions of the eye.

Typical for its function, the eye is well accessible to light and the ophthalmologist uses light for investigational purposes like the slit lamp biomicroscope to obtain visual information on the health and integrity of the ocular tissue. Several diagnostic techniques using light are available including specular microscopy(2) and confocal microscopy(3) to study the cornea cell layers, cornea topography(4) to measure the curvature of the cornea, and fluorescein angiography(s) to study the retinal blood flow and to detect alterations in fluid dynamics. The laser flare meter(6) gives information on the amount of total proteins in the anterior chamber, optical coherence tomography(7) enables the visualization and measurement of different eye structures, the laser scanning ophthalmoscope ${ }^{(8)}$ provides information on retinal blood flow or optic nerve alterations, and the retinal thickness analyzer provides information on retinal and macular thickness $(9)$. Other types of radiation $(1,10)$ that can be used for diagnostic means, 
however not restricted to the eye, include ultrasound, magnetic resonance imaging, and computed tomography.

Quantitative information on tissue composition or chemical substances however, can be obtained only by invasive techniques such as collecting material by biopsy or needle aspiration and investigating the samples in vitro. Obtaining the information noninvasively would have several advantages including limiting the risk of spreading of contagious diseases, preventing infection (endophthalmitis) to the eye under investigation, and rapid onsite information. A noninvasive technique could follow up on treatment techniques like photodynamic therapy (PDT) in case of age-related macular degeneration, laser coagulation for retinopathies, or after retina surgery. Spectroscopy, or the interaction of light with any matter(11-12), offers possibilities to achieve this goal. Spectroscopic techniques have been employed to several tissues(10,13-14). Light that interacts with matter can be absorbed, emitted or scattered(11,15). Today, absorption, fluorescence and scattering spectroscopies are most extensively used.

Raman spectroscopy(16), a technique dependent on the scattering of radiation, allows the investigation of molecules in aqueous environments where, due to the poor infrared transmittance of water, absorption spectroscopy is less useful. Raman spectroscopy, needing a strong monochromatic light source, made a revival after the introduction of the laser in 1960 and the development of more sensitive photon detectors(17) in the 1970's. Annually, a considerable amount of literature related to Raman spectroscopy is released(18-20) and there are numerous applications of Raman spectroscopy(21-22) in medical, biological, technical and environmental disciplines.

\section{Methods}

The aim of this paper is to give an overview of the different applications of Raman spectroscopy in ophthalmology after a brief introduction of the Raman effect with references to handbooks, general articles and some examples of specific applications. The sources for the search were Medline, Current contents, and also patents accessible on the Internet. A short introduction with relevant anatomical and structural information precedes the specific eye structure that will be reviewed.

\section{The Raman effect and instrumentation}

The Raman effect(16) occurs when photons of incident light interact with molecules in either a gas, liquid or solid material. The difference in the photon energy between the scattered light and the incident light results from the change in the vibrational or rotational state of the molecule. This is an inelastic scattering process since the scattered light can have a lower (Stokes Raman scattering) or a higher (anti-Stokes Raman scattering) frequency than the incident light. This differs from Rayleigh scattering, where the incident and scattered photons have the same frequency (same energy, elastic scattering event). The Raman shift, usually expressed in wavenumber $\left(\mathrm{cm}^{-1}\right)$ units on the $x$-axis of a recorded spectrum, is independent of the exciting wavelength. However, the intensity ( $y$-axis of a recorded spectrum) of the Raman scattered light does depend on both the wavelength and intensity of the incident light. The Raman intensity is about $10^{-6}$ to $10^{-8}$ times that of Rayleigh scattering. 


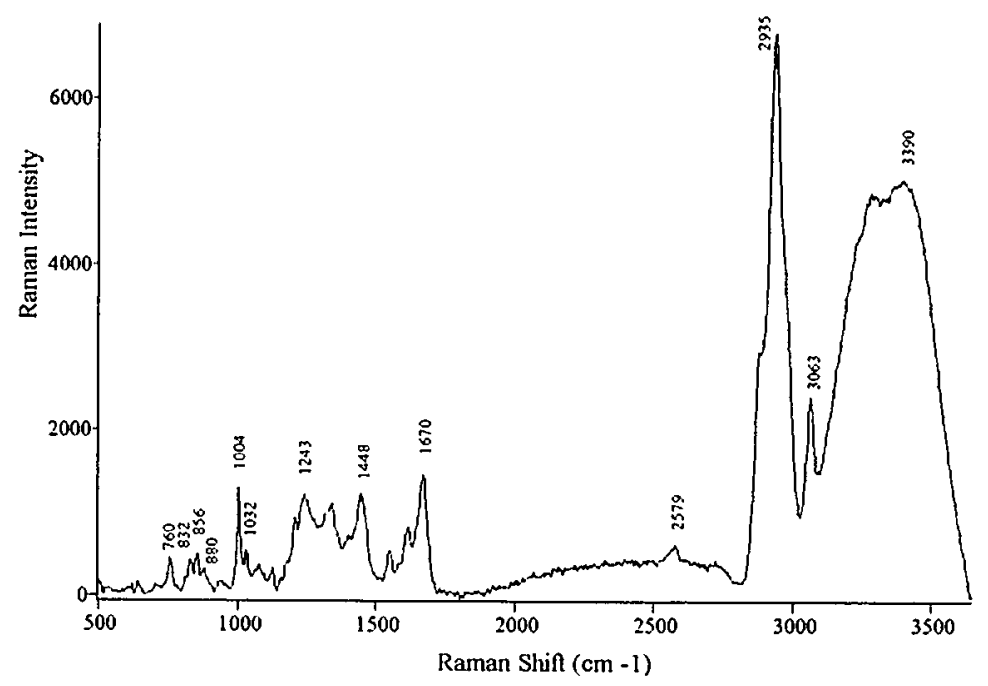

Fig. 2. Raman spectrum of rabbit lens in vitro. The peak assignments shown are not exhaustive since these are for demonstration purposes. The excitation source was an Argon laser emitting at 514.5 $\mathrm{nm}$ with a $25 \mathrm{~mW}$ power setting and an integration time of 4 seconds. As can be seen the peak intensities differ in amplitude.

Figure 2 shows a typical Raman spectrum from a rabbit lens in vitro. The $\mathrm{OH}$ mode from water at $3390 \mathrm{~cm}^{-1}$ is strong but fortunately located at the far end of the spectral region, well separated from other vibrational modes corresponding to different chemical substances. In a lens, the protein concentration is high, which is reflected in a strong $\mathrm{C}-\mathrm{H}$ mode signal at 3063 and $2935 \mathrm{~cm}^{-1}$, while an Amide I mode is located at $1670 \mathrm{~cm}^{-1}$ and the Amide III mode at $1243 \mathrm{~cm}^{-1}$. Amino acids can be seen: Phenylalanine at 1032 and $1004 \mathrm{~cm}^{-1}$; and tyrosine and tryptophan in the 'aromatic' region at $880,856,832$ and $760 \mathrm{~cm}^{-1}$. The spectral region between 1800 and $2500 \mathrm{~cm}^{-1}$ is fairly inactive regarding Raman activity.

Another type of inelastic scattering is fluorescence, which may interfere with the Raman signal(23-25). Fluorescence is generally a stronger and broader spectral peak in comparison to Raman scattering, which consists of sharp but weak spectral peaks.

Since the discovery of the Raman effect, further explorations have yielded new insights into the physics of the Raman effect. Examples are resonance Raman(26) spectroscopy (resonance Raman spectroscopy occurs if the exciting frequency approaches or coincides with an excited electronic state of the molecule) and Raman Optical Activity (ROA)(27) techniques (ROA employs the chiral properties of molecules). Other developments involve refinement in technical and software techniques like Fourier Transform Raman spectroscopy where an interferometer and a mathematical model are incorporated(28-29). Further development of the laser (30-31) has made different excitation modalities possible: Tunable lasers may be used in two wavelength excitation techniques; the narrow bandwidth of the laser makes resonance Raman spectroscopy easier to work with; optical nonlinear effects can be utilized; and timeresolved spectroscopy can be applied. Several authors(32-33) have investigated the possibili- 
ties of labeling a biological molecule with a Raman active molecule. These new discoveries broadened the uses of Raman spectroscopy, even into the field of ophthalmology, where safe low-level excitations are mandatory in order to prevent tissue damage.

To generate a Raman spectrum, a monochromatic light source with sufficient photon density is required. The Raman intensity is inversely proportional to the fourth power of the incident light wavelength $\left(I=\propto / \lambda^{4}\right)$. Before the development of the laser, obtaining Raman spectra was a cumbersome procedure requiring large sample volumes and very long collecting times (up to $24 \mathrm{hrs}$.). With lasers it was possible to obtain Raman spectra from very small volumes of either gases, liquids or solids(28).

Another challenge was the detection of the faint Raman intensities. Now, obtaining Raman spectra with exposure times in the order of milliseconds is possible due to the high quantum efficiency of the Charge Coupled Device (CCD) detectors, while covering a broad frequency range (17). They are sensitive between $200-1100 \mathrm{~nm}$ with a maximum quantum efficiency (i.e., the probability of a photon to generate a single free electron in the pixel it impacts) between $300-800 \mathrm{~nm}$. The development of a CCD detector with a maximum quantum efficiency around $900 \mathrm{~nm}$ would be interesting since the Raman spectra at longer wavelengths are further away from the possible fluorescence excitation in biological samples and it avoids sample degradation (34). However, recalling that the Raman intensity is inversely proportional to the fourth power of the incident light wavelength, choosing a longer excitation wavelength will strongly reduce the Raman scattering, emphasizing the delicate balance between the excitation source and the detector. The choice of a photosensor depending on the application has been reviewed(35-36). The biggest challenge to applying Raman in the living tissue is obtaining a sufficient signal- to-noise ratio spectrum without damage to the tissue.

Raman spectroscopy can be performed using fiber optics, and the development of probes made it possible to use Raman in a remote fashion. A fiber optic based probe(37) was designed to monitor chemical processes in ovens. However, to obtain Raman spectra from living cells or tissues in situ, a confocal design of the probes is essential to limit the depth of field and to exclude detection of the fluorescence from other parts in the samples(38-39). In the Raman experiment, Rayleigh scattering from the tissue needs to be rejected before the Raman signal is directed into the spectrometer. In triple grating spectrometers, the first two gratings serve to reject the Rayleigh scattering. However, the disadvantage of the triple grating spectrometer is that the Raman signal throughput is about $12.5 \%$. In single grating spectrometers, the throughput is about $50 \%$. Here, the Rayleigh rejection is achieved by several types of filters like Chevron filters, crystalline colloidal filters and holographic notch filters ${ }^{(40-42)}$. Of course, the spectroscopist also enjoys the progress(29.43) in mechanical/electronical design (accurate steppen motor control), optical design (holographic gratings, holographic notch filters, light guides), digital data acquisition and computer processing modalities (controlling the spectrometer, processing and presenting data, neural networks).

These analyses can also be conducted in a biological environment, and several handbooks have been written including topics on this subject(23-24). In Chapter 11 of reference(25), Huong gives an overview for biological applications of Raman spectroscopy with examples involving vision, carcinogenesis and neural transmission.

Patents, accessible on the Internet(44), are another source of information on spectroscopic techniques. The search results on 'Raman spectroscopy' gave 1008 patents. Mahadevan- 


\section{Focussed on}

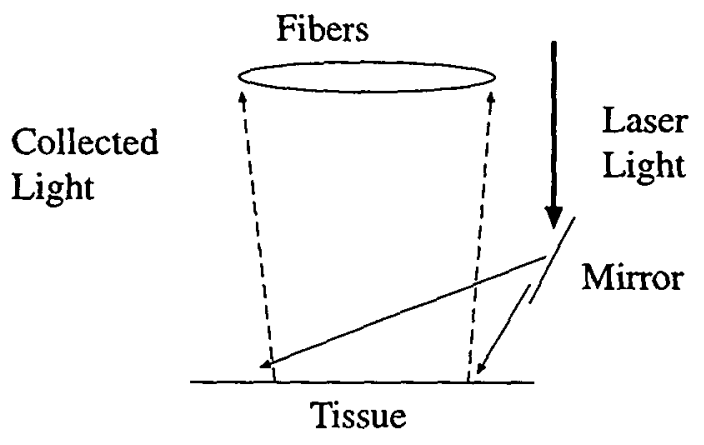

Fig. 3. Off axis illumination according to reference ${ }^{(45)}$.

Jansen et al. describes in a patent(45) a setup for in vivo measurements of Raman signals. They use a contact probe placed on the tissue as shown in Fig. 3.

With the Raman spectroscopic technique it is possible to analyze organic and/or inorganic solids, liquids and gases, either qualitative or quantitative(28). Small samples can be studied like chromosomes(38) and conformation of DNA(46), where it has been found that there was a difference in Raman spectra of the DNA in the native state or in aqueous solution (relaxed conformation). Proteins have been extensively studied with Raman spectroscopy(25,47-49). It provides information on the peptide backbone, detection and geometry of disulfide bonds, sulfhydryl groups and methionine residues. Ring-stretching vibrations of aromatic residues from phenylalanine, tryptophan, tyrosine and histidine give rise to high Raman intensities, and this suggests that these groups can be detected even in low concentrations. The use of Raman spectroscopy in enzymology has been reviewed(50).

FT-Raman was used to study several applications in medicine including comparison of normal and pathological tissues(51). An example of forensic/archeological use of the Raman technique was the investigation of a small skin tissue specimen (16.2 $\mathrm{mg}$ ) from the 'Iceman', a late Neolithic man found between Italy and Austria in a glacier in 1991(52). Currently, Raman spectroscopy is clinically used for monitoring respiratory and anesthetic gases in the operating room(53).

In ophthalmology, the use of Raman spectroscopy is a challenge as Raman techniques require a large amount of light, which easily can damage delicate eye tissues. However, the use of sophisticated optics in conjunction with sensors with a high quantum detection efficiency makes in vivo applications of Raman spectroscopy feasible. To extract the information of a chosen sub-volume, two kinds of collection modalities could be employed. One can isolate a specific volume by selective illumination, for instance by a slit lamp (Fig. 4A). In a backscatter design, one can prevent out-of-focus radiation reaching the detector by a pinhole in a confocal setup (Fig. 4B). The selective illumination technique implies that the illumination axis and the collection axis are at an angle. The closer the collection angle approaches 90 degrees with respect to the illumination axis, the better the tissue can be inspected. Limitations of the maximum achievable angle are set by the mismatch of refraction between air and the precorneal tear film, by the diameter of the clear cornea and by the 


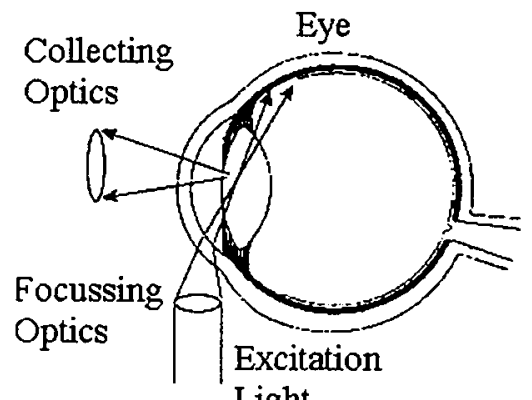

Light

Fig. 4A. Selective illumination.

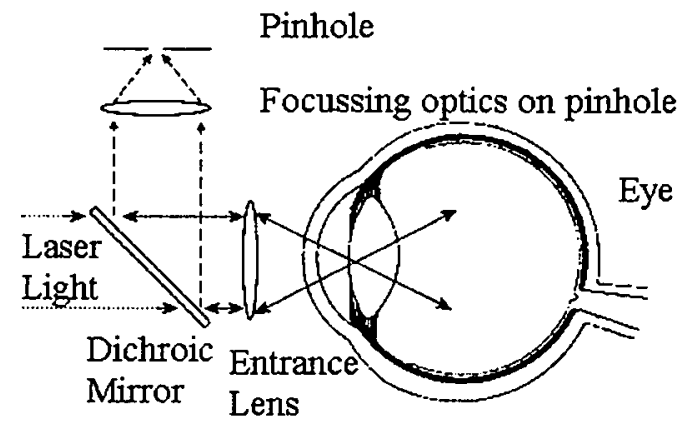

Fig. 4B. Confocal design.

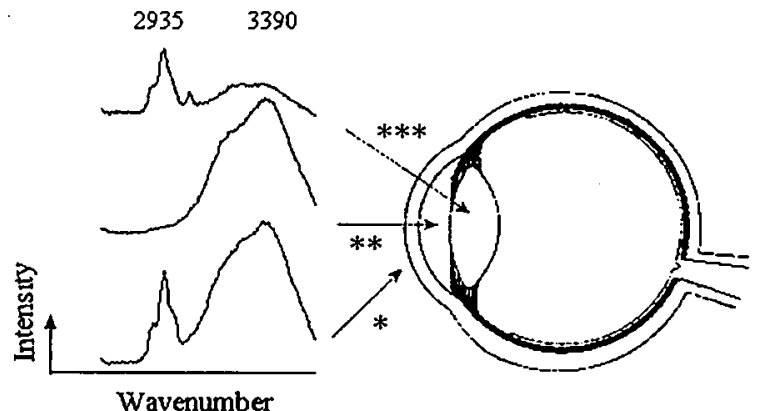

Raman spectra of eye tissues:

* Cornea

** Aqueous humor

**** Lens

Fig. 4C. Raman spectra of eye tissue. Experimental settings: Argon laser $514.5 \mathrm{~nm}$ with a power setting of $5 \mathrm{~mW}$ and an integration time of 1 second. 
pupil aperture diameter. A typical example for optical sectioning is the slit lamp used by almost every ophthalmologist. Samuels et al.(54) describes in a patent a setup for measuring characteristics of biological tissues including the eye. The collection of photons occurs in an off-axis configuration. Tarr et al.(55) shows in a patent a setup for noninvasive blood glucose measurements using stimulated Raman spectroscopy. They employ a forward scatter configuration, i.e., the excitation beam enters at the peripheral cornea, crosses the anterior chamber and leaves at the opposite peripheral cornea. However, due to the index of refraction mismatch at the entrance air/precorneal tear film interface and at the exit precorneal tear film/air interface in the non-contact mode, clinical use may be limited. To obtain a detailed image of the lens or the corpus vitreous, dilation of the pupil is essential in most cases. In the case of collecting photons for spectroscopic purposes, it is clear that maintaining alignment during the measurement is very difficult if movements are to be encountered or if the tissue needs to be scanned.

Backscatter facilitates the use of an entrance lens that not only delivers the exciting light but also collects the scattered light, thus eliminating an alignment procedure between the excitation and collection focus. However, since the two axes are the same, light scattering originating from the anterior and posterior tissues located in the probing beam axis will both contribute to the collected signal. Hence, exact determination of the position is difficult. A solution to this problem is a confocal design that limits the depth of field, by excluding signals (e.g. fluorescence) of the adjacent tissue and allows for exact tissue location.

Comparing the selective illumination technique with the backscatter technique brings the differences into view. The selective illumination method requires a cumbersome alignment procedure due to differences in the index of refraction between the probing and collecting path. These displacements and distortions make it difficult to let the foci of the probing and collecting beam coincide. The confocal approach allows the adjustment of the depth of field and determination of tissue location; alignment is fixed, and hence the index of refraction has little effect on alignment. The rejection of Rayleigh and fluorescence scattering is critical in both techniques.

Typical Raman spectra obtained from in vivo rabbit eyes with the confocal setup, while scanning through the different ocular tissues, are shown in Fig. 4C. A variety of proteins can be measured. In this example, the long spectral range is shown, where the carbon-hydrogen (CH) stretching mode at $2935 \mathrm{~cm}^{-1}$ and the hydroxyl $(\mathrm{OH})$ stretching mode at $3390 \mathrm{~cm}^{-1} \mathrm{can}$ be seen. As can be expected in this spectral region, the aqueous humor exhibits the $\mathrm{OH}$ mode from water as main Raman feature.

\section{Results}

Scientific literature on Raman spectroscopy in ophthalmology.

The different eye structures will be discussed progressing from outer (comea) to inner (retina). Fig. 1 shows a drawing of the human eye. So far the most investigated structures using Raman spectroscopy are the lens and the retina. 


\section{Cornea and sclera}

The cornea and sclera form the globe of the eye, also known as fibrous tunic. The most anterior part of the eye, located in the palpebral fissure is the cornea. It is a clear avascular structure with a central thickness of about $580 \mu \mathrm{m}$, increasing to about $700 \mu \mathrm{m}$ in the peripheral region. The anterior part (epithelium) is $50 \mu \mathrm{m}$ thick, the intermediate part (stroma) includes more than $90 \%$ of the total thickness and the posterior part (endothelium) is only $5 \mu \mathrm{m}$ thick. Located on the epithelium layer is the tear film which, because of its large index of refraction with respect to air, accounts for about $70 \%$ of the refractive power of the unaccomodated eye.

Goheen et al.(56) studied feline corneal collagen after aging, heating or drying. Aged or heat treated corneas showed broader Amide I and III bands.

A Fourier transform Raman spectroscopic study by Mizuno et al.(57) in vitro of intact rabbit corneas and scleras showed spectroscopic features from the collagen proteins. A microRaman spectroscopic study of organ-cultured corneas was performed by Siew et al. (58) to investigate if quality control of these eye bank corneas was feasible using this technique. It proved to be a sensitive technique for measurements in a non-contact fashion. Studies on contact lenses have been performed by various groups(59-61).

Many drugs are applied to the eye for different applications. A drug used to treat glaucoma (dorzolamide) was applied to the rabbit comea in vivo by Bauer et al.(62) for quantification of the transport of the drug through the cornea (pharmacokinetics). The same group ${ }^{(63)}$ studied axial cornea hydration in phantom media and in vivo rabbit corneas using the $\mathrm{CH} / \mathrm{OH}$ Raman signals arising from the protein/water constituents of the cornea tissue. An attempt to measure cornea hydration in patients performed by Bauer et al.(64) was successful, however due to unsafe laser excitation energy, only legally blind patients could be included in this study. For this experiment, a backscatter setup as shown in Fig. 4 B was used.

\section{Aqueous humor}

The aqueous humor is fundamental to the health of the eye. It supplies the lens and comea with sodium, potassium, oxygen and glucose. In the normal eye, it contains more than $98 \%$ water; hence, the concentration of biological molecules is less than $2 \%$. It contains almost no proteins $(5-16 \mathrm{mg} / 100 \mathrm{ml})$. Consequently, Raman spectroscopic features of these substances are considerably weaker than that of water and, thus, more difficult to detect. Wang et al.(65) showed that at biological concentrations in simulated and in vitro aqueous humor samples, Raman scattering from these molecules scatter light independently of each other and the scattering intensity is linearly proportional to their concentrations. Wicksted et al.(66) showed that these measurements could be conducted on both human and rabbit aqueous humor specimens in vitro using considerably lower laser powers with shorter exposure times and at different wavelengths to reduce fluorescence when CCD detectors are used. Erckens et al.(67) discussed an optical technique where Raman scattering could be used to characterize ocular tissues with the potential of detecting biological molecules within the aqueous humor in vivo. The need to improve the analysis of Raman spectra was addressed in several papers(68-70) $u$ tilizing multivariate calibration techniques of Partial-Least Squares applied to simulated rabbit aqueous humor specimens with physiologic levels of glucose and other biological molecules, respectively. 


\section{Lens}

The transparent lens, positioned between the aqueous humor and the corpus vitreous, accounts for the remaining $30 \%$ of the eye's refractive power and it is responsible for the accommodation (focusing) of the eye. It has a high protein content (about 34\%) but with an uneven distribution through the lens, reaching maximum values in the dense nuclear region. Changes in protein and water content especially in the elderly population may form opacities referred to as 'cataract'.

Due to the high protein content, the lens is suitable for investigation with Raman spectroscopy(71-74). However, the strong fluorescence, especially in the cataractous lens, will demand an extra effort in limiting the influence of these unwanted photons on the Raman signal. Using a Raman microprobe, fluorescence studies on human eye lenses could be carried out by Barron et al.(75). Also the distribution of metabolic related fluorophors in human lenses could be studied by Yu et al.(76). By slicing lenses, Bot et al.(77) were able to collect Raman signals even from a cataract lens. By homogenizing and preparing animal or human lenses, surface enhanced Raman analysis(78-79) could be carried out (fluorescence is quenched). By using Near Infrared Fourier Transform Raman Spectroscopy, Nie et al.(80) showed that fluorescence could be limited.

\section{In vitro studies:}

Research on chemical or structural characterization.

In 1975 Yu et al.(71) designated in bovine lenses the water soluble proteins as $\alpha-, \beta 1-, \beta 2-$, $\beta 3-$, and $\gamma$-crystallin. Each exhibits different vibrational frequencies.

Schachar et al. (72) states that lens proteins are organized in an antiparallel $\beta$-pleated sheet. Raman was used by East et al.(81) in the rat and mouse model to show the conversion of the sulfhydryl group to disulfide during aging of the nucleus. The tyrosine/phenylalanine ratio remained undisturbed in the nucleus, so the amino acid composition does not change. However, in the cortex, with aging of the lens, the tyrosine/phenylalanine ratio changed from 1.6 to 0.8 . This correlates with the increasing proportion of $\alpha$-crystallin and the decreasing proportion of $\gamma$-crystallin. Also, the irradiation with long wavelength ultraviolet light decreases the number of -SH and S-S bonds(82). Baranska et al.(83) studied intermolecular interactions in animal lenses. Local protein content in porcine lenses is described by de Korte et al.(84). Yaroslavsky et al.(85) performed combined elastic and Raman light scattering of human eye lenses in vitro. Borchman et al.(86-88) studied lipid composition of human lens epithelium, cortex and nucleus. Vrensen et al.(89) described maturation of human fiber membranes. Paterson et al.(90) described membrane structures in lenses. Borchman et al.(91-92) and Sato et al.(93) studied lipid membranes in lenses. Schyns et al.(94) studied paraformaldehyde fixation and lens proteins. Tomohiro et al.(95) showed in hydrogen peroxide treated rat lens that in the cortex sulfhydryl groups were decreased and disulfide bonds were increased. Shun-Shin et al.(96) studied Christmas tree cataracts in human donor eyes and revealed that cystine is the most likely candidate for the formation of the Christmas tree needles. Smeets et al.(97) described an age-related increase in tryptophan (ratios integrated peak $760: 1450 \mathrm{~cm}^{-1}$ ) in the human lens core as an increase in the $\beta$-sheet signals in the Amide I and II bonds. Chiou et al.(98) determined in pigeon eye lenses that the secondary structures of $\alpha$ - and $\beta$ crystallins are mainly anti-parallel beta-pleated sheet in nature. Calf gamma crystallins were described(99-100). Castillo et al.(101) confirmed a high concentration of glycogen in lenses of the 
ring-neck dove and the pigeon. Yu et al.(102) demonstrated the presence of a carotenoid in the lens of the deep-sea hatchetfish using resonance Raman.

The water content of the lens has been studied since this plays a major role in lens transparency. It was found by Huizinga et al.(103) that the local variations in absolute water content could be measured in human and rabbit eye lenses. Iriyama et al.(104) found in the cacstrain mouse lenses lens water changes during cataractogenesis. The ratio of the $\mathrm{OH}$ intensity from water at $3390 \mathrm{~cm}^{-1}$ and the $\mathrm{CH}$ intensity from proteins at $2935 \mathrm{~cm}^{-1}$ were used to observe the changes in the water content.

Research on the development of experimentally induced cataracts.

Nakamura et al.(105) found no significant difference in the intensity ratios of several key Raman bands in mouse perforated eyes. Dai et al.(106) found water imbibition in the lens nuclear region of galactose induced cataract in a mouse model. Horikiri et al.(107) induced a cataract in the rat lens using sodium selenite and observed an age dependent decrease in water and decrease of $\mathrm{SH}$ residues in the normal animal, while in the cataract animals the water content was 2-3 times higher and the value of the SH decrease was lower. Toshima et al.(108) found an increase in cortical and nuclear water after streptozotocin induced cataract in the rat lens. Zigman et al.(109) observed a loss of sulfhydryl compounds after ambient exposure to near-ultraviolet radiation in gray squirrel lens. Cai et al.(110) detected changes in sulfhydryl and water in galactose-induced cataracts in the rat lens. Takise et al.(111) studied Raman spectra of rabbit lenses after irradiation with an ultraviolet laser beam. Mizuno et al.(112) found no significant change in the relative water content or secondary structures of the proteins in the glucocorticoid-induced cataract in the chick embryo. However the intensity ratios of the tyrosine doublet bands were changed. Barron et al.(113) found that the loss of SH can be accelerated in vivo by long wavelength UV exposure in the guinea pig lens. Thomas et al.(114) obtained Raman spectra of normal and cataractous rabbit lenses induced by ultraviolet radiation. Nozawa et al.(115) and Mizuno et al.(116) studied the effect of the aldose reductase inhibitor on experimental diabetic cataracts. Mizuno et al.(117) found lens hydration in response to chronic hyperglycemic stress mainly in the cortical region. Treatment with the aldose reductase inhibitor Ponalrestat suppressed lens hydration in cortical and nuclear regions in similar diabetic rats. Bertoluzza et al.(118) observed a decrease of the fluorescence in the Raman spectrum in vitro from human treated cataractous lenses with an anticataract drug. Ozaki et al.(119) studied in lens aging inter and intramolecular disulfide bond formation and structural changes in lens proteins.

Ondruska et al.(120) investigated several animal lenses and formation of dry and cold cataracts. Mizuno et al.(121) studied in cold cataracts (appears in nucleus below $26^{\circ} \mathrm{C}$ ) the tyrosine doublet and observed a change in the hydrogen bonding environment.

Research on senile cataracts. Several groups studied protein changes due to age in different models(113,122-125). Yu et al.(126) studied fluorophors in mice lenses, and found evidence for metabolic production of a green fluorophor(127) in human lenses. Duindam et al.(128) studied in vitro focal opacities in human lenses and found protein changes, disruption of fiber membranes and formation of vesicles from the membrane constituents. Duindam et al.(129) described confocal Raman microspectroscopy combined with filipin (binds to cholesterol) to detect cholesterol in the femtogram range and showed(130) with the probe filipin age-related changes of the 3-beta-OH-cholesterol distribution along the optical axis in human eye lenses. Barron et al.(131) studied tryptophan in guinea pig lenses. Ozaki et al.(132) compared 
mouse, rat, rabbit and guinea pig lens nuclei at various stages of aging and reported different rates of dehydration, inter and intramolecular disulfide bond formation and microenvironmental changes in the tryptophan residues. Siebinga et al.(133) studied protein confirmation in human lenses age 20-75 years with these conclusions. Nuclear proteins contain more aromatic amino acids like tryptophan, tyrosine and phenylalanine which are not age dependent although they appear more abundant in 'young' nuclear proteins; and proteins localized in early small opacities do not show alterations in conformation. In another study Siebinga et al.(134) proved that the mean nuclear water content increases with age in human lenses. Askren et al.(135) obtained an -SH concentration profile along the visual axis of rat and mouse lenses. Kuck et al.(136) studied sulfhydryl concentration in different species and concluded that lenses can be divided in two groups: those with rapid loss of -SH during aging (rodent) and those that maintain -SH well (human, bovine). Mizuno et al.(137) found a loss of sulfhydryl (SH) groups and an increase in disulfide (S-S) bonds in aging lenses. Ozaki et al. (138) found a decrease in tryptophan residue in lens aging in the mouse lens.

Research on hereditary cataracts. Iriyama et al.(139) and Itoh et al.(140) studied Raman bands as diagnostic markers for cataract formation. Mizuno et al.(141) detected in the Shumiya Cataract Rat (hereditary induced cataract, manifest in nuclear and perinuclear parts of the lens at 11 weeks of age) a decreased level of protein disulfide bonds and an increase of protein sulfhydryl groups. Mizuno et al.(142) suggested that the relative water content in the lens was not a precataractous sign for the ICR/f-strain rat.

In situ studies: Mathies et al.(143) obtained Raman spectra of good quality with an intensified Vidicon detector and long ( $>10$ minutes) integration time of intact bovine lenses with $1 \mathrm{~mW}$ of $514.5 \mathrm{~nm}$ radiation. Intact rabbit and rat lenses were measured intraocularly by Mizuno et al.(144). The development of a high-fidelity Raman imaging microscope by Goldstein et al.(145) offers potential in situ applications to investigate the lens. Compatibility studies or structure determination of artificial intraocular lenses (IOL) implanted after cataract extraction. Galin et al.(146) studied unpolymerized methylmethacrylate in IOL's. Legeais et al.(147) studied Teflon coated IOL's and used, among other techniques, Raman spectroscopy for an elemental composition check after removal of the IOL. Bertoluzza et al.(148) and Smit et al.(149) studied the different IOL types in vitro using Raman spectroscopy. Bertoluzza et al.(150) investigated the structural properties of polymers used in ophthalmology.

In vivo animal study: The first in vivo Raman spectrum of the cortex and nuclear region of a rabbit eye lens in the $500-1750 \mathrm{~cm}^{-1}$ wavenumber range was obtained by Yu et al.(151) in 1982. The optical sectioning technique as shown in Fig. 4A was used.

In vivo human study: Erckens et al.(152) determined the IOL type in patients using an energy of only $95 \mu \mathrm{J}$ to obtain a spectrum using the confocal approach as depicted in Fig. 4B.

\section{Corpus vitreous}

The corpus vitreous is a clear gel composed mainly of water (99\%). It acts as a supporting structure and a metabolic pathway for nutrients for the lens and retina. The clarity of the vitreous body may be compromised by traumatic or pathologic events.

Sebag et al.(153) found in vitreous, obtained from patients after vitrectomy, two Raman peaks at $1604 \mathrm{~cm}^{-1}$ and at $3057 \mathrm{~cm}^{-1}$ which were more pronounced in the diabetic retinopathy group than in the patients group without a diabetic retinopathy. 


\section{Retina}

The refractive power of the anterior located eye structures focus the light onto a small region on the retina, the fovea. About $10 \%$ of the light incident on the cornea reaches the photoreceptors, while the rest is lost due to scattering and absorption in the ocular media, blood vessels and neural cells of the retina. The thickness of the retina is between 0.1 and $0.5 \mathrm{~mm}$ depending on the location. Retinal tissue is made of light sensitive neural cells that convert light into an electric signal that is transported to the brain by means of the optic nerve. The light that can reach the retina has a wavelength between 400 and $1400 \mathrm{~nm}$. There are two types of light sensitive cells with different properties: Rods and cones. Rods have a pigment, rhodopsin, that perceives light with an absorbance maximum around $496 \mathrm{~nm}$ and function especially for dim light vision. Cones have pigments, iodopsins, which absorb different wavelengths. There are "blue", "green" and "red" cones with an absorbance maximum at about $425 \mathrm{~nm}, 530 \mathrm{~nm}$, and $560 \mathrm{~nm}$ respectively, which facilitate the perception of colors. The visual pigments are made of opsin (an apoprotein) and an 11-cis-retinal chromophore. The 11-cis-retinal chromophore is bound to the opsin by a protonated Schiff base (PBS) linked to a specific lysine residue (Lys296). In vertebrates, the absorption maximum of the retinal chromophore can be tuned over a fairly large range, from about 360 to $635 \mathrm{~nm}$. This is called the opsin shift. When excited with light, the femtosecond isomerization of the chromophore is initiated which leads to a hyperpolarization of the photoreceptor cell membrane and creation of a visual impulse. So, the phototransduction in rods starts with the absorption of light by rhodopsin, which initiates a cascade of events with numerous discrete steps and occurring in very short time intervals with short living chemical bonds. These mechanisms have been widely investigated by several groups using different types of Raman spectroscopy. Lewis(154) used tunable resonance Raman spectroscopy to study the role of rhodopsin in transducing light energy into a chemical reaction. Oseroff et al.(155) used resonance Raman spectroscopy to study rhodopsin in retinal disk membranes. Rothschild et al.(156-157) obtained resonance and nonresonance Raman spectra from photoreceptor membranes. Doukas et al.(158) studied bovine metharhodopsin I and II. Marcus et al.(159) studied the retinylidene chromophore in bacteriorhodopsin and other intermediates. Smith et al.(160) determined the retinal chromophore structure in bacteriorhodopsin. Fodor et al.(161) obtained the structure of the retinal chromophore in the $\mathrm{hR}_{\mathrm{L}}$ intermediate of halorhodopsin. Vogel et al.(162) determined the secondary structure of bacteriorhodopsin. $\mathrm{Ujj}_{\mathrm{jj}}$ et al.(163) used picosecond time resolved anti-Stokes coherent Raman spectroscopy to record the vibrational spectrum of the lumi-rhodopsin intermediate formed in the microsecond time frame of the room temperature rhodopsin (RhRT) photoreaction. Huang et al.(164) found some differences in the $\mathrm{C}=\mathrm{C}$ stretch mode between bovine and octopus rhodopsin and bathorhodopsin. Yoshizawa et al.(165) studied the photochemical properties of chicken iodopsin and compared them with rhodopsin and found the following: The regeneration rate of iodopsin with 11-cis-retinal was 240 times faster than rhodopsin; meta-iodopsin II decayed about 100 times faster than metarhodopsin II. Palings et al.(166) studied hydrogen out-of-plane wagging vibrations in bathorhodopsin. Pande et al.(167) investigated octopus rhodopsin, and studied in UV-sensitive insect eyes visual pigments(168). Kochendoerfer et al.(169) studied the wavelength regulation mechanism in human visual pigments. Palings et al.(170) investigated the fingerprint vibrations in rhodopsin, bathorhodopsin and isorhodopsin. Mathies(171) gave an overview on the molecular picture of the primary events in vision. Popp et al.(172-173) studied with picosecond 
resonance coherent anti-Stokes Raman scattering vibrational spectra of rhodopsin and bathorhodopsin, and performed a vibrational analysis from bacteriorhodopsin containing isotopically substituted retinal chromophores(174).

The visual pigments in different species have been investigated. Lin et al.(175) identified 12 amino acid residues in human blue cone pigment that might be related to the green to blue opsin shift. In chicken cone cells Lin et al.(176) investigated the molecular mechanism of the opsin shift. Kochendoerfer et al.(177) examined the molecular basis of the spectral tuning. Loppnow et al.(178) studied the retinal chromophore in 7,9-dicis-rhodopsin. Loppnow et al.(179) demonstrated that a blue visual pigment contains an unperturbed protonated Schiff base. Barry et al.(180) concluded that the differences of the Raman spectra of A-1 and A-2 pigments were caused by some intrinsic difference of the retinal chromophores. Barry et al.(181182) studied Raman vibrational spectra from single photoreceptor cells. Eyring et al.(183) studied visual pigment analogues to assess different models for the structure of the retinal chromophore in bathorhodopsin. The primary photochemical events in visual pigments have been studied(184-187).

The Schiff base. Eyring et al.(188) found evidence for a protonated Schiff base linkage in bathorhodopsin. Deng et al.(189) studied the Schiff base in bovine rhodopsin, bathorhodopsin, and isorhodopsin. Abdulaev et al.(190) used surface-enhanced Raman spectra in determining the distance between the retinal Schiff base and the cytoplasmic side of native disk. Deng et al.(191) found evidence for a bound water molecule next to the retinal Schiff base in bacteriorhodopsin and rhodopsin. Jager et al.(192) investigated photolysis intermediates of rhodopsin Schiff base counterion mutants.

The dynamics of the isomerization. Kochendoerfer et al.(193) studied the role of intramolecular steric interactions in the isomerization of the 11-cis-retinal chromophore in the photoreceptor protein rhodopsin. Loppnow et al.(194) determined the change in geometry upon electronic excitation of rhodopsin's 11-cis-retinal protonated Schiff base. Liu et al.(195) proposed a model for the primary process of vision involving a concerted-twist motion. Hayward et al.(196) suggested that the isomerization is complete within picoseconds after the absorption of a photon. Zhu et al.(197) demonstrated that the trans-bathorhodopsin retinal chromophore is completely formed in $<2 \mathrm{ps}$ after photoexcitation.

In the research for age-related macular degeneration, macular carotenoid levels were measured by Bernstein et al.(198) not only in flat mounted human retina and in intact animal eyes using resonance Raman scattering, but also in the living human(199) using a backscatter design.

\section{Conclusion}

Since the invention of the laser in 1960, a variety of Raman techniques have been developed. The modality to detect low light level signals facilitated the investigation of the distinct structures from the eye in vitro and in vivo in an animal model and even in human eyes (see Fig. 5.). The eye, due to its optical properties, is well suited to optical spectroscopic techniques, which can probe the intact tissue layers of the eye. In vitro Raman experiments showed several interesting aspects worthy of further investigation in vivo. A major research effort is on cataract formation since this is still a large cause of vision disturbances in the world. In cataract formation, the water content and distribution through the lens play a key 


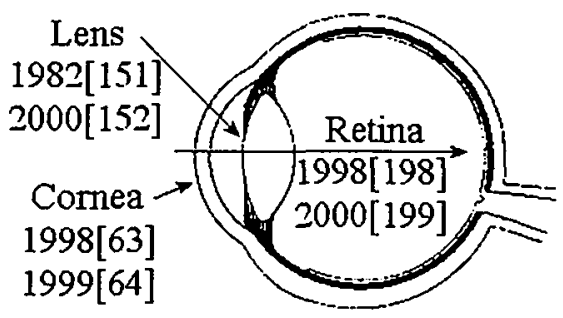

Fig. 5. In vivo obtained Raman spectra of different ocular tissues as described in the literature.

role as do amino acids and protein conformations. The 'quest' is finding the cause of cataract, and for this, in vivo techniques could reveal the different steps and changes in the lens. Raman spectroscopy is a unique tool in lens research since it provides structural information(81-99) on proteins, amino acids (like Try, Trp, Phe), and sulphur bonds (-SH, S-S). The maturation process of the proteins in the lens cortex and nucleus can be studied in the intact lens, thus preventing oxidation of sulfhydryls and protein disruption. Protein confirmation studies ${ }^{(133)}$ in human lens revealed that nuclear proteins contain more aromatic amino acids. This technique was also used to study parts of the lens, which revealed a regional swelling in diabetic rats(116117), or detection of focal opacities in human lenses with protein changes(128). The finding that cystine is the most likely candidate(96) for the Christmas tree needles in a Christmas tree cataract, is an example of using Raman spectroscopy to reveal the structure and/or cause of a pathologic condition. These findings justify the exploration of the Raman spectroscopic technique for use in vivo. Yu et al.(151) obtained the first in vivo Raman spectrum in a rabbit and proved the feasibility of the Raman technique to pursue in vivo applications. Erckens et al.(152) obtained the first Raman spectrum of an IOL in a patient using a retina safe excitation power setting. The increased application of biomaterials and vitreous humor substitutes in the eye make research efforts on biocompatibility, safety and endurance of these materials mandatory. Here, Raman spectroscopy can provide such information either in vitro or in vivo.

Comparatively less explored are the aqueous humor and the corpus vitreum. Both tissues have a low concentration of biological molecules in common but are of paramount importance to the integrity and health of the eye. If the aqueous humor could be studied in vivo with Raman spectroscopy, a far more accurate diagnosis could be performed compared with the now commonly used slit lamp examination, e.g. revealing only a "negative" or "positive" Tyndall (light scattering in the aqueous humor). The corpus vitreum is located behind the lens and is adjacent to the retinal tissue. This location makes it a more difficult target for in vivo Raman spectroscopy. Possibly, semi-invasive techniques using fiber guided Raman signals, e.g. as reported by Schoen et al.(37), can be modified for intraocular use. It would also be possible during intraocular surgery (vitrectomy) to obtain Raman spectra by means of the intraocular instruments to evaluate the tissue or determine molecular structures. The retina tissue has been studied in vitro to identify the ultrafast events leading to the visual impulse(171), and the visual pigments(177). The results by Bernstein et al.(198-199) open a fascinating field in the detection of the macular pigments, which could be a step in understanding, and hopefully early detection and treatment of macular degeneration, a leading cause of blindness in the elderly.

However, for introducing the Raman technique as a clinically applied diagnostic tool, much 
more work has to be done. The use of Raman spectroscopy in animal research may reduce the number of animals needed, especially in longitudinal studies. Also, if Raman spectra can be obtained in a noncontact mode, the anesthesia and data collection may become less eventful for the animal.

Looking for possible improvements at the discrete stages of the Raman method—an adequate light source; knowledge about the optical properties of tissue (light-matter interaction); the optics for collecting and transport of the light; detection devices; and (mathematical) evaluation - one can conclude that little gain is to be expected from the light source, some from the detection devices, but considerable gain can be expected from the light-matter interaction and the optics and the mathematical analysis of the Raman data.

Considering the valuable insights already gained with Raman spectroscopic techniques and the possibilities for further refinements and developments, it can be concluded that its role in biomedical applications has introduced a new era in noninvasive and nondestructive techniques for tissue determination.

\section{References}

1. Duane's Clinical Ophthalmology. Lippincott-Raven Publishers, Hagerstown MD 1997.

2. Sugar A. Clinical specular microscopy. Surv. Ophthalmol 1979;24:21-32.

3. Minsky M. Memoir on inventing the confocal scanning microscope. Scanning 1988;10:128-38.

4. Jongsma FH, de Brabander J, Hendrikse F. Review and classification of comeal topographers. Lasers Med Sci 1999;14:219.

5. Novorny HR, Alvis DL. A method of photographing fluorescence in circulating blood of the human retina. Circulation $1961 ; 24: 82$.

6. Sawa M. Clinical application of laser flare-cell meter. Jap. J. Ophthal. 1990:34:346-363.

7. Huang D, Swanson E, Lin CP et al. Optical coherence tomography. Science 1991;254:1178-81.

8. Webb R. Scanning Laser Ophthalmoscope. IEEE Transact Biomed Eng 1981:28:488-492.

9. Landau D, Schneidman E, Jacobovitz T, Rozenman Y. Quantitative in vivo retinal thickness measurements in healthy subjects. Ophthalmology 1997;104:639-642.

10. Masters B. Noninvasive diagnostic techniques in Ophthalmology. New York: Springer-Verlag, 1990.

11. Ditchbum R. Light. Dover publications, Mineola NY 1991.

12. Skoog D, Leary J. Principles of Instrumental Analysis (Fourth Edition). Saunders College Publishing 1992 Orlando FL.

13. McCully $K$, Mancini D, Levine S. Nuclear magnetic resonance spectroscopy: its role in providing valuable insight into divers clinical problems. Chest 1999;116:1434-41.

14. de Graaf, R. In vivo NMR Spectroscopy. John Wiley \& Sons 1998 Chisester, West Sussex, England.

15. Ramaseshan S. Scientific papers of C.V. Raman, Volume 1: Scattering of light. Indian Academy of Sciences in Bangalore; 1988.

16. Raman C, Kirishnan K. A new type of secondary radiation. Nature. 1928;121:105.

17. Boyle WS, Smith GE. Charge Coupled Semiconductor Devices. Bell Systems Technical Journal 1970;49:587-593.

18. Gerrard, D. Raman Spectroscopy. Anal. Chem. 1994:66:547R-557R.

19. Lyon L. Keating C, Fox A, Baker B, He L. Nicewarner S, Mulvaney S, Natan M. Raman Spectroscopy. Anal. Chem. 1998:70:341R-361R.

20. Mulvaney S, Keating C. Raman Spectroscopy. Anal. Chem. 2000;72:145R-157R.

21. Carey PR. Raman spectroscopy, the sleeping giant in structural biology, awakes. J Biol Chem 1999;274:26625-8. 
22. Hanlon EB, Manoharan R. Ko TW, Shafer KE, Motz JT, Fitzmaurice M et al. Prospects for in vivo Raman spectroscopy. Phys Med Biol 2000;45:R1-59.

23. Carey PR. Biomedical Applications of Raman and Resonance Raman Spectroscopies. New York: Academic Press, 1982.

24. Tu AT. Raman spectroscopy in biology. New York: John Wiley \& Sons, 1984.

25. Grasselli $G$ and Bulkin B. Analytical Raman Spectroscopy. Chemical Analysis series, vol 114. New York: John Wiley \& Sons, 1991.

26. Carey PR. Resonance Raman spectroscopy in biochemistry and biology. Quarterly Reviews of Biophysics 1978;11:309370.

27. Barron L, Hecht L. Bell A. Raman optical activity: an incisive new probe of the structure and dynamics of biomolecules. Science Progress 1998:81:17-34.

28. Gardiner DJ and Graves PR. Practical Raman Spectroscopy, Berlin: Springer-Verlag, 1989.

29. Chase B. A new generation of Raman instrumentation. Applied Spectroscopy 1994:48(7):14A-19A.

30. Evans T. Applications of lasers to chemical problems. Techniques of Chemistry Vol XVII, John Wiley \& Sons, New York NY, 1982.

31. Hilinski E, Rentzepis P. Biological applications of picosecond spectroscopy. Nature 1983;302:481-487.

32. Sijtsema N, Duindam J, Puppels G. Otto C, Greve J. Imaging with extrinsic Raman labels. Applied Spectroscopy 1996;50:545-551.

33. Carey P. Resonance Raman labels and Raman labels. J. Raman Spectroscopy 1998:29:861-868.

34. Puppels GJ, Olminkhof JH. Segers-Nolten GM, Otto C, de Mul FF, Greve J. Laser irradiation and Raman spectroscopy of single living cells and chromosomes: sample degradation occurs with $514.5 \mathrm{~nm}$ but not with $660 \mathrm{~nm}$ laser light. Exp Cell Res 1991:195:361-7.

35. Campion A. Woodruff W. Multichannel Raman spectroscopy. Anal. Chemistry 1987;59:1299A-1308A.

36. Niemczyk T, Delgado-Lopez M, Newman C. Multichannel Raman spectroscopy tackles industrial problems. Laser Focus World 1993:March:85-98.

37. Schoen C, Cooney T, Sharma S, Carey D. Long fiber-optic remote Raman probe for detection and identification of weak scatterers. Applied Optics. 1992;31:7707-7715.

38. Puppels G, de Mul F, Otto C. Greve J. Robert-Nicoud M, Anndt-Jovin D, Jovin T. Studying single living cells and chromosomes by confocal Raman microspectroscopy. Nature. 1990;347:301-303.

39. Jongsma F, Erckens R, Wicksted J, Bauer N, Hendrikse F, March W, Motamedi M. Confocal Raman spectroscopy system for noncontact scanning of ocular tissues: An in vitro study. Optical Engineering 1997;36:3193-3199.

40. Battey D, Slater J, Wludyka R, Owen H, Pallister D. Morris M. Axial transmissive f/1.8 imaging Raman spectrograph with volume-phase holographic filter and grating. Applied Spectroscopy. 1993:47:1913-1919.

41. Kim M, Owen H. Carey P. High-performance Raman spectroscopic system based on a single spectrograph. CCD, notch filters, and a $\mathrm{Kr}^{+}$laser ranging from the near-IR to near-UV regions. Applied Spectroscopy. 1993;47:1780-1783.

42. Puppels G, de Grauw C, te Plate M. Greve J. Chevron-type dielectric filter set for efficient narrow-band laser line rejection in Raman microspectrometers. Applied Spectroscopy. 1994;48:1399-1402.

43. Goldner H. Dr. Raman's legacy shines with renewed interest. R\&D Magazine 1992;July:56-58.

44. USA Patent Server, www-web (Intemet) address:http://www.uspto.gov

45. Mahadevan-Jansen A. Richards-Kortum R, Mitchell M. USA Patent Number 5.842,995, Dec. 1. 1998.

46. Peticolas W, Patapoff T, Thomas G, Postlewait J, Powell J. Laser Raman microscopy of chromosomes in living eukaryotic cells: DNA Polymorphism in vivo. Joumal of Raman Spectroscopy 1996:27:571-578.

47. Lord R. Yu N. Laser-excited Raman spectroscopy of biomolecules I. J. Mol. Biol. 1970:50:509-524.

48. Lord R, Yu N. Laser-excited Raman spectroscopy of biomolecules II. J. Mol. Biol. 1970;51:203-213

49. Lord R. Strategy and tactics in the Raman spectroscopy of biomolecules. Applied Spectroscopy 1977:31:187-194.

50. Carey P. Raman spectroscopy in enzymology: the first 25 years. J. Raman Spectroscopy 1998;29:7-14. 
51. Keller S. Schrader B. Hoffmann A, Schrader W. Metz K. Budach W et al. Application of Near-Infrared-Fourier Transform Raman spectroscopy in medical research. Joumal of Raman Spectroscopy 1994;25:663-671.

52. Williams AC, Edwards HG, Barry BW. The 'Iecman': molecular structure of 5200-year-old skin characterised by Raman spectroscopy and electron microscopy. Biochim-Biophys-Acta 1995;1246(1):98-105.

53. Westenskow D, Smith K, Coleman D, Gregonis D, van Wagenen R. Clinical evaluation of a Raman scattering multiple gas analyzer for the operating room. Anesthesiology 1989:70:350-355.

54. Samuels M, Patterson S, Eppstein J, Yu NT, Bursell S. Apparatus and methods for measuring characteristics of biological tissues and similar materials. USA Patent Number 5582,168, Dec 10, 1996.

55. Tarr R, Steffes P. Non-invasive blood glucose measurement system and method using stimulated Raman spectroscopy. USA Patent Number 5,243,983, Sep. 14, 1993.

56. Goheen S, Lis L, Kauffman J. Raman spectroscopy of intact feline comeal collagen. Biochimica et Biophysica Acta 1978;536:197-204.

57. Mizuno A, Tsuji M, Fujii K. Kawauchi K. Ozaki Y. Near-infrared Fourier transform Raman spectroscopic study of comea and sclera. Jpn J Ophthalmol. 1994:38:44-8.

58. Siew D. Clover G, Cooney R, Wiggins P. Micro-Raman spectroscopic study of organ cultured comeae. J. Raman Spectroscopy 1995;26:3-8.

59. Hosala S, Ozawa H, Tanzawa H, Ishida H, Yoshimura K, Momose T, Magatani H, Nakajima A. Analysis of deposits on high water content contact lenses. J Biomed Mater Res. 1983 Mar.17(2):261-74.

60. Kantarci Z. Aksoy S, Hasinci N. Estimation of monomer content in polymethyl methacrylate contact lens materials by Raman spectroscopy. Int J Artif Organs. 1997;20:407-11.

61. Monti P, Simoni R, Caramazza R. Bertoluzza A. Applications of Raman spectroscopy to ophthalmology: spectroscopic characterization of disposable soft contact lenses. Biospectroscopy. 1998;4:413-9.

62. Bauer N.J.C., Motamedi M., Wickisted J.P., March W.F., Webers CAB, Hendrikse F. Noncontact assessment of ocular pharmacokinetics using confocal Raman spectroscopy. J Ocular Pharm Ther 1999;15:123-134.

63. Bauer N.J.C., Wicksted J.P., Jongsma F.H.M., March W.F., Hendrikse F. and Motamedi M. Noninvasive assessment of the hydration gradient across the comea using confocal Raman spectroscopy. Invest. Ophthalmol. Vis. Sci. 1998:39,831.

64. Bauer N.J.C., Hendrikse F., March W.F. In vivo confocal Raman spectroscopy of the human comea. Comea 1999;18:483488.

65. Wang SY, Hasty CE, Watson PA, Wicksted JP, Stith RD, March WF. Analysis of metabolites in aqueous solutions by using laser Raman spectroscopy. Appl. Opt. 1993;49:987-993.

66. Wicksted JP, Erckens RJ, Motamedi M, March WF. Raman spectroscopy studies of metabolic concentrations in aqueous solutions and aqueous humor specimens. Applied Spectroscopy 1995;49:987-993.

67. Erckens RJ, Wicksted JP, March WF, Motamedi M. Raman spectroscopy for non-invasive characterization of ocular tissue: Potential for detection of biological molecules. Journal of Raman Spectroscopy 1997:28:293-299.

68. Goetz MJ. Cote GL. Erckens RJ. March WF, Motamedi M. Application of a Mulivariate technique to Raman spectra for quantification of body chemicals. IEEE Trans. Biomed. Eng. 1995;42:728-731.

69. Lambert J, Storrie-Lombardi M, Borchert M. Measurement of physiologic glucose levels using Raman spectroscopy in a rabbit aqueous humor model. LEOS Newsletter 1998;12:19-22.

70. Borchert M, Storrie-Lombardi, Lambert J. A noninvasive glucose monitor: Preliminary results in rabbits. Diabetes Technology \& Therapeutics 1999;1:145-151.

71. Yu NT, East E. Laser Raman spectroscopic studies of ocular lens and its isolated protein fractions. The Joumal of Biological Chemistry 1975;250(6):2196-2202.

72. Schachar RA, Solin SA. The microscopic protein structure of the lens with a theory for cataract formation as determined by Raman spectroscopy of intact bovine lenses. Invest Ophthalmol. 1975;14:380-96.

73. Yu NT, East EJ, Chang RC, Kuck JF. Raman spectra of bird and reptile lens proteins. Exp Eye Res. 1977;24:321-34. 
74. Yu NT. Raman spectroscopy: a conformational probe in biochemistry. CRC Crit Rev Biochem. 1977;4:229-80.

75. Barron BC, Yu NT, Kuck JF Jr. Distribution of a 488.0-nm-excited fluorophor in the equatorial plane of the human lens by a laser Raman microprobe: a new concept in fluorescence studies. Exp Eye Res. 1988:47:901-4.

76. Yu NT, Barron BC. Kuck JF Jr. Distribution of two metabolically related fluorophors in human lens measured by laser microprobe. Exp Eye Res. 1989;49:189-94.

77. Bot A. Huizinga A. De Mul F, Vrensen G. Greve J. Raman Microspectroscopy of Fixed Rabbit and Human Lenses and Lens Slices: New Potentialities. Exp. Eye Res. 1989;49;161-169.

78. Nie S, Castillo C, Bergbauer K, Kuck J, Nabiev I, Yu NT. Surface-enhanced Raman spectra of eye lens pigments. Applied Spectroscopy 1990;44:571-575.

79. Sokolov K, Lutsenko S, Nabiev I, Nie S, Yu NT. Surface-enhanced Raman analysis of biomedical eye lens extracts. Applied Spectroscopy 1991:45:1143-1148.

80. Nie S, Bergbauer K, Kuck J, Yu NT. Near Infrared Fourier Transform Raman Spectroscopy in Human Lens Research. Exp. Eye Res. 1990;51(5):619-623

81. East EJ, Chang RC, Yu NT, Kuck JF Jr. Raman spectroscopic measurement of total sulfhydryl in intact lens as affected by aging and ultraviolet irradiation. Deuterium exchange as a probe for accessible sulfhydryl in living tissue. J Biol Chem. 1978;253:1436-41.

82. Yu NT, DeNagel DC. Pruett PL, Kuck JF Jr. Disulfide bond formation in the eye lens. Proc Natl Acad Sci U S A. 1985:82:7965-8.

83. Baranska H, Labudzinska A. Study of NH stretching band in Raman spectra of animal lenses. Lens Eye Toxic Res 1991;8:189-94.

84. De Korte CL, Van Der Steen AF, Thijssen JM, Duindam JJ, Otto C, Puppels GJ. Relation between local acoustic parameters and protein distribution in human and porcine eye lenses. Exp Eye Res. 1994;59:617-27.

85. Yaroslavsky IV, Yaroslavsky AN. Otto C, Puppels GJ, Vrensen GF, Duindam H, Greve J. Combined elastic and Raman light scattering of human eye lenses. Exp Eye Res. 1994;59:393-9.

86. Borchman D, Lamba OP, Ozaki Y, Czamecki M. Raman structural characterization of clear human lens lipid membranes. Curr Eye Res 1993;12:279-84.

87. Borchman D, Ozaki Y, Lamba OP, Byrdwell WC, Czamecki MA, Yappert MC. Structural characterization of clear human lens lipid membranes by near-infrared Fourier transform Raman spectroscopy. Curr Eye Res 1995;14:511-5.

88. Borchman D, Tang D, Yappert MC. Lipid composition, membrane structure relationships in lens and muscle sarcoplasmic reticulum membranes. Biospectroscopy 1999;5:151-67.

89. Vrensen GF, Duindam HJ. Maturation of fiber membranes in the human eye lens. Ultrastructural and Raman microspectroscopic observations. Ophthalmic Res 1995:27 Suppl 1:78-85.

90. Paterson CA, Zeng J. Husseini Z, Borchman D, Delamere NA, Garland D, Jimenez-Asensio J. Calcium ATPase activity and membrane structure in clear and cataractous human lenses. Curr Eye Res. 1997;16:333-8.

91. Borchman D, Ozaki Y, Lamba OP, Byrdwell WC, Czameckj MA, Yappert MC. Structural characterization of clear human lens lipid membranes by near-infrared Fourier transform Raman spectroscopy. Curr-Eye-Res. 1995;14:511-5.

92. Borchman D, Tang D, Yappert MC. Lipid composition, membrane structure relationships in lens and muscle sarcoplasmic reticulum membranes. Biospectroscopy. 1999:5:151-67.

93. Sato H, Borchman D, Ozaki Y, Lamba OP, Byrdwell WC, Yappert MC, Paterson CA. Lipid-protein interactions in human and bovine lens membranes by Fourier transform Raman and infrared spectroscopies. Exp Eye Res. 1996;62:47-53.

94. Schyns MW, Huizinga A. Vrensen GF, de Mul FF. Greve J. Paraformaldehyde fixation and some characteristics of lens proteins as measured by Raman microspectroscopy. Exp Eye Res 1990;50:331-3.

95. Tomohiro M, Mizuno A. Alteration of lens sulfhydryl groups induced by oxidative stress: Raman spectroscopic study of hydrogen peroxide-treated rat lens. Jpn J Ophthalmol 1995:39:130-6. 
96. Shun-Shin G, Vrensen G, Brown N, Willekens B, Smeets M. Bron A. Morphologic characteristics and chemical composition of Christmas tree cataract. Invest Ophthalmol Vis Sci 1993;34:3489-96.

97. Smeets M, Vrensen G, Otto K, Puppels G, Greve J. Local Variations in Protein Structure in the Human Eye Lens: A Raman Microspectroscopic Study. Biochimica et Biophysica Acta. 1993:1164:236-242.

98. Chiou SH, Chen W. Structural analysis of pigeon lens crystallins by near-infrared Fourier transform Raman spectroscopy. Biochem Int 1992;28:401-12.

99. Yu NT, DeNagel DC, Slingsby C. Raman spectroscopy of calf lens gamma-II crystallin: direct evidence for the formation of mixed disulfide bonds with 2-mercaptoethanol and glutathione. Exp Eye Res. 1989;48:399-410.

100. Pande J, McDermot MJ, Callender RH. Spector A. The calf gamma crystallins-a Raman spectroscopic study. Exp Eye Res. 1991:52:193-7.

101. Castillo CG, Lo WK, Kuck JF, Yu NT. Nature and localization of avian lens glycogen by electron microscopy and Raman spectroscopy. Biophys J 1992;61:839-44.

102. Yu NT, Cai MZ, Lee BS, Kuck JF Jr, McFall-Ngai M, Horwitz J. Resonance Raman detection of a carotenoid in the lens of the deep-sea hatchetfish. Exp Eye Res 1991;52:475-9.

103. Huizinga A, Bot A, de Mul F, Vrensen G, Greve J. Local variation in absolute water content of human and rabbit eye lenses measured by Raman microspectroscopy. Exp. Eye Res. 1989;48:487-496.

104. Iriyama K, Mizuno A, Ozaki Y, Itoh K, Matsuzaki H. An application of laser Raman spectroscopy to the study of a hereditary cataractous lens; on the Raman band for a diagnostic marker of cataractous signatures. Curr Eye Res. 1982/83;2:48992.

105. Nakamura K, Jung YM, Era S, Sogami M, Ozaki Y, Takasaki A. 1H-NMR and Raman studies on perforating traumainduced cataract formation in a mouse lens. Biochim Biophys Acta 2000;1474:23-30.

106. Dai S, Qi S, Zhang L, Bai C. Ni T, Deng X. Laser Raman spectrometry study on experimental galactose-induced cataract. Yen-Ko-Hsueh-Pao. 1995;11:143-6.

107. Horikiri K, Nakajima H, Matsuura T, Narama I, Fujimoto Y, Ozaki Y. Estimation of structural changes in the cataractous rat lens using Raman spectroscopy. Exp.Anim. 1992;41:225-230.

108. Toshima S, Miyazaki H, Mizuno A. Raman study of the lenses of spontaneously-occurring and streptozotocin-induced diabetic rats. Jpn J Ophthalmol 1990;34:436-41.

109. Zigman S, Paxhia T. McDaniel T, Lou MF, Yu NT. Effect on chronic near-ultraviolet radiation on the gray squirrel lens in vivo. Invest Ophthalmol Vis Sci 1991;32:1723-32.

110. Cai MZ, Kuck JF Jr, Yu NT. Galactose-induced cataract in rat: Raman detection of sulfhydryl decrease and water increase along an equatorial diameter. Exp Eye Res 1989:49:531-41.

111. Takise S, Horiguhi S, Fukumura H, Hayashi K, Miki T, Fukumoto K, Yamashita H, Yoshikawa S. Karai I, Harima M. Morphological change and Raman specurum of rabbit lens irradiated with ultraviolet laser beam. Osaka City Med J. 1989:35:29-37.

112. Mizuno A, Nishigori H, Iwatsuru M. Glucocorticoid-induced cataract in chick embryo monitored by Raman spectroscopy. Invest Ophthalmol Vis Sci. 1989;30:132-7.

113. Barron BC. Yu NT, Kuck JF Jr. Raman spectroscopic evaluation of aging and long-wave UV exposure in the guinea pig lens: a possible model for human aging. Exp Eye Res. 1988;46:249-58.

114. Thomas DM, Schepler KL. Raman spectra of normal and ultraviolet-induced cataractous rabbit lens. Invest Ophthalmol Vis Sci. 1980;19:904-12.

115. Nozawa H, Yaginuma T, Mizuno A. Raman spectroscopic study of the effect of aldose reductase inhibitor on experimental diabetic cataract. Nippon Ganka Galkkai Zasshi. 1988;92:194-201.

116. Mizuno A. Nozawa H, Yaginuma T, Matsuzaki H, Ozaki Y, Iriyama K. Effect of aldose reductase inhibitor on experimental diabetic cataract monitored by laser Raman spectroscopy. Exp Eye Res. 1987;45:185-6.

117. Mizuno A. Toshima S and Mori Y. Confirmation of lens hydration by Raman spectroscopy. Exp. Eye Res. 1990;50:647-9. 
118. Bertoluzza A. Fagnano C. Monti P. Caramazza R, Cellini M. Raman spectra of the human lens in relation to pathology and the anticataract effect of drugs. J. Raman Spectroscopy 1986:17:133-137.

119. Ozaki Y, Mizuno A, Itoh K, Iriyama K. Inter- and intramolecular disulfide bond formation and related structural changes in the lens proteins. A Raman spectroscopic study in vivo of lens aging. J Biol Chem. 1987 15;262:15545-51.

120. Ondruska O, Hanson DM. Raman spectra of duck, rat, and flounder lenses and the formation of dry and cold cataracts. Exp Eye Res. 1983;37:139-43.

121. Mizuno A. Ozaki Y. Itoh K. Matsushima S. Iriyama K. Raman spectroscopic evidence for the microenvironmental change of some tyrosine residues of lens proteins in cold cataract. Biochem Biophys Res Commun. 1984 30;119:989-94.

122. Ozaki $Y$ and Mizuno A. Molecular aging of lens crystallins and the life expectancy of the animal. Age-related protein structural changes studied in situ by Raman spectroscopy. Biochimica et Biophysica Acta. 1992;1121:245-251.

123. Kuck JF Jr, Yu NT. Raman and fluorescent emission of the human lens. A new fluorophor. Exp Eye Res. 1978:27:737-41.

124. Yu NT, Kuck JF Jr, Askren CC. Red fluorescence in older and brunescent human lenses. Invest Ophthalmol Vis Sci. 1979;18:1278-80.

125. Yu NT, Bando M, Kuck JF Jr. Fluorescence/Raman intensity ratio for monitoring the pathologic state of human lens. Invest Ophthalmol Vis Sci. 1985;26:97-101.

126. Yu NT, Bando M, Kuck JF Jr. Metabolic production of a blue-green fluorophor in lenses of dark-adapted mice and its increase with age. Invest Ophthalmol Vis Sci. 1983;24:1157-61.

127. Yu NT, Cai MZ, Ho DJ, Kuck JF Jr. Automated laser-scanning-microbeam fluorescence/Raman image analysis of human lens with multichannel detection: evidence for metabolic production of a green fluorophor. Proc Natl Acad Sci U S A. 1988;85:103-6.

128. Duindam JJ, Vrensen GF, Otto C, Greve J. Cholesterol, phospholipid, and protein changes in focal opacities in the human eye lens. Invest Ophthalmol Vis Sci 1998;39:94-103.

129. Duindam HJ, Vrensen GF, Otro C, Puppels GJ, Greve J. New approach to assess the cholesterol distribution in the eye lens: confocal Raman microspectroscopy and filipin cytochemistry. J Lipid Res 1995;36:1139-46.

130. Duindam JJ, Vrensen GF, Otto C. Greve J. Aging affects the conformation of cholesterol in the human eye lens. Ophthalmic Res 1996;28 Suppl 1:86-91.

131. Barron BC, Yu NT, Kuck JF Jr. Tryptophan Raman/457.9-nm-excited fluorescence of intact guinea pig lenses in aging and ultraviolet light. Invest Ophthalmol Vis Sci. 1987:28:815-21.

132. Ozaki Y. Mizuno A. Molecular aging of lens crystallins and the life expectancy of the animal. Age-related protein structural changes studied in situ by Raman spectroscopy. Biochim Biophys Acta 1992;1121:245-51.

133. Siebinga I, Vrensen GF, Otto K, Puppels GJ, De Mul FF, Greve J. Ageing and changes in protein conformation in the human lens: a Raman microspectroscopic study. Exp Eye Res 1992;54:759-67.

134. Siebinga I, Vrensen GF, De Mul FF, Greve J. Age-related changes in local water and protein content of human eye lenses measured by Raman microspectroscopy. Exp Eye Res 1991;53:233-9.

135. Askren CC, Yu NT, Kuck JF Jr. Variation of the concentration of sulfhydryl along the visual axis of aging lenses by laser Raman optical dissection technique. Exp Eye Res. 1979;29:647-54.

136. Kuck JF, Yu NT, Askren CC. Total sulfhydryl by Raman spectroscopy in the intact lens of several species: variations in the nucleus and along the optical axis during aging. Exp Eye Res. 1982;34:23-37.

137. Mizuno A, Ozaki Y. Aging and cataractous process of the lens detected by laser Raman spectroscopy. Lens Eye Toxic Res 1991;8:177-87.

138. Ozaki Y, Mizuno A, Itoh K, Yoshiura M, Iwamoto T, Iriyama K. Raman spectroscopic study of age-related structural changes in the lens proteins of an intact mouse lens. Biochemistry. 1983;22:6254-9.

139. Iriyama K, Mizuno A, Ozaki Y, Itoh K and Matsuzaki H. An application of laser Raman spectroscopy to the study of a hereditary cataractous lens; on the Raman band for a diagnostic marker of cataractous signatures. Current Eye Research 1982/1983;2:489-492. 
140. Itoh K, Ozaki Y, Mizuno A, Iriyama K. Structural changes in the lens proteins of hereditary cataracts monitored by Raman spectroscopy. Biochemistry. 1983:22:1773-8.

141. Mizuno A, Shumiya S, Toshima S, Nakano T. Alteration of lens disulfide bonds in newly developed hereditary cataract mat. Jpn J Ophthalmol 1992:36:417-25.

142. Mizuno A. Kanematsu EH, Suzuki H. Ihara N. Laser Raman spectroscopic study of hereditary cataractous lenses in ICR/fstrain rat. Jpn J Ophthalmol. 1988;32:281-7.

143. Mathies R, Yu NT. Raman spectroscopy with intensified vidicon detectors: A study of intact bovine lens proteins. Journal of Raman Spectroscopy 1978:7:349-352.

144. Mizuno A. Ozaki Y, Kamada Y. Miyazaki H, Itoh K, Iriyama K. Direct measurement of Raman spectra of intact lens in a whole eyeball. Curr Eye Res 1981/82:1:609-13.

145. Goldstein SR, Kidder LH, Herne TM. Levin IW, Lewis EN. The design and implementation of a high-fidelity Raman imaging microscope. J Microsc. 1996; 184:35-45.

146. Galin MA. Turkish L. Chowchuvech E. Detection, removal, and effect of unpolymerized methylmethacrylate in intraocular lenses. Am J Ophthalmol. 1977:84:153-9.

147. Legeais JM, Wemer LP, Legeay G, Briat B, Renard G. In vivo study of a fluorocarbon polymer-coated intraocular lens in a rabbit model. J Cataract Refract Surg 1998;24:371-9.

148. Bertoluzza A, Fagnano C. Monti P. Semerano G, Garcia-Ramos J, Caramazza R, Cellini M. Raman spectra of intraocular lenses before and after implantation in relation to their biocompatibility. J. Raman Spectroscopy 1987;18:151-152.

149. Smit EE, Erckens RJ, Hendrikse F, Motamedi M, Wicksted JP, March WF. Identification of intraocular lens materials using confocal Raman spectroscopy. J Cataract Refract Surg. 1999;25:1498-504.

150. Bertoluzza A, Monti P, Arciola CR, Pizzoferrato A. Structural properties and biocompatibility of polymers used in ophthalmology. Clin Mater. 1991;8:165-9.

151. Yu NT. Kuck J. Askren C. Laser Raman Spectroscopy of the Lens in situ, measured in an anesthetized rabbit. Curr Eye Res 1982;1:615-618.

152. Erckens RJ. March WF, Jongsma FJ. Wicksted JP. Hendrikse F, Smit EE, Motamedi M. Noninvasive Raman spectroscopic identification of the intraocular lens material in the living human eye. In press in the Joumal of Cataract and Refractive Surgery.

153. Sebag J, Nie S. Reiser K, Charles MA, Yu NT. Raman spectroscopy of human vitreous in proliferative diabetic retinopathy. Invest Ophthalmol Vis Sci. 1994:35:2976-80.

154. Lewis A. Tunable laser resonance Raman spectroscopic investigations of the transduction process in vertebrate rod cells. Fed Proc. 1976;35:51-3.

155. Oseroff AR, Callender RH. Resonance Raman spectroscopy of thodopsin in retinal disk membranes. Biochemistry. 1974;13:4243-8.

156. Rothschild KJ, Andrew JR, De Grip WJ, Stanley HE. Opsin structure probed by Raman spectroscopy of photoreceptor membranes. Science. 1976;191:1176-8.

157. Rothschild KJ, Stanley HE. Models of ionic transport in biological membranes. Raman spectroscopy as a probe of valinomycin, gramicidin $A^{\prime}$, and thodopsin conformations. Am J Clin Pathol, 1975:63:695-713.

158. Doukas AG, Aton B, Callender RH. Ebrey TG. Resonance Raman studies of bovine metharhodopsin I and metharhodopsin II. Biochemistry. 1978;17:2430-5.

159. Marcus $M$, Lewis A. Resonance Raman spectroscopy of the retinylidene chromophore in bacteriorhodopsin $\left(b R_{570}\right), b R_{560}$ $\mathrm{M}_{\mathbf{4 1 2}}$, and other intermediates: Structural conclusions based on kinetics, analogues. models, and isotopically labeled membranes. Biochemistry 1978;17:4722-4735.

160. Smith SO, Lugtenburg J, Mathies RA. Determination of retinal chromophore structure in bacteriorhodopsin with resonance Raman spectroscopy. J Membr Biol. 1985;85:95-109. 
161. Fodor S, Bogomolni R, Mathies R. Structure of the retinal chromophore in the hRL intermediate of halorhodopsin from resonance Raman spectroscopy. Biochemistry 1987;26:6775-6778.

162. Vogel H, Gartner W. The secondary structure of bacteriorhodopsin determined by Raman and circular dichroism spectroscopy. The Journal of Biological Chemistry 1987; 262:11464-11469.

163. Uji L, Jager F, Atkinson GH. Vibrational spectrum of the lumi intermediate in the room temperature rhodopsin photo-reaction. Biophys J. 1998;74:1492-501.

164. Huang L, Deng H, Koutalos Y, Ebrey T, Groesbeek M. Lugtenburg J, Tsuda M. Callender RH. A resonance Raman study of the $\mathrm{C}=\mathrm{C}$ stretch modes in bovine and octopus visual pigments with isotopically labeled retinal chromophores. Photochem Photobiol. 1997;66:747-54.

165. Yoshizawa T, Imamoto Y. Structure and photobleaching process of chicken iodopsin. Biophys Chem. 1995:56:57-62.

166. Palings I, van den Berg EM. Lugtenburg J, Mathies RA. Complete assignment of the hydrogen out-of-plane wagging vibrations of bathorhodopsin: chromophore structure and energy storage in the primary photoproduct of vision. Biochemistry. 1989:28:1498-507.

167. Pande C, Pande A, Yue K. Callender R, Ebrey T, Tsuda M. Resonance Raman spectroscopy of octopus rhodopsin and its photoproducts. Biochemistry 1987;26:4941-4947.

168. Pande C. Deng H, Rath P, Callender RH, Schwemer J. Resonance Raman spectroscopy of an ultraviolet-sensitive insect hodopsin. Biochemistry. 1987;26:7426-30.

169. Kochendoerfer GG, Wang Z, Oprian DD, Mathies RA. Resonance Raman examination of the wavelength regulation mechanism in human visual pigments. Biochemistry 1997:36:6577-6587.

170. Palings I, Pardoen JA, van den Berg E, Winkel C, Lugtenburg J, Mathies RA. Assignment of fingerprint vibrations in the resonance Raman spectra of rhodopsin, isorhodopsin, and bathorhodopsin: implications for chromophore structure and environment. Biochemistry. 1987:26:2544-56.

171. Mathies RA. Photons, femtoseconds and dipolar interactions: a molecular picture of the primary events in vision. Novartis Found Symp. 1999;224:70-84; discussion 84-101.

172. Popp A, Ujj L, Atkinson GH. Vibrational spectra of room-temperature thodopsin: concentration dependence in picosecond resonance coherent anti-Stokes Raman scattering. Biophys Chem. 1995;56:129-35.

173. Popp A, Ujj L, Atkinson GH. Bathorhodopsin structure in the room-temperature rhodopsin photosequence: picosecond time-resolved coherent anti-Stokes Raman scattering. Proc Natl Acad Sci U S A. 1996:93:372-6.

174. Popp J, Popp A, Ujj L, Atkinson G, Sheves M, Ottolenghi M. Vibrational analysis of Coherent Anti-Stokes Resonance Raman spectra from bacteriothodopsin containing isotopically substituted retinal chromophores. J. Raman Spectroscopy 1996:27:87-95.

175. Lin SW, Kochendoerfer GG, Carroll KS, Wang D, Mathies RA, Sakmar TP. Mechanisms of spectral tuning in blue cone visual pigments. Visible and Raman spectroscopy of blue-shifted thodopsin mutants. J Biol Chem 1998b;273:24583-24591.

176. Lin SW, Imamoto Y, Fukada Y, Shichida Y, Yoshizawa T, Mathies RA. What makes red visual pigments red? A resonance Raman microprobe study of retinal chromophore structure in iodopsin. Biochemistry 1994;33:2151-2160.

177. Kochendoerfer GG, Lin SW, Sakmar TP, Mathies RA. How color visual pigments are tuned. Trends Biochem Sci. 1999;24:300-5.

178. Loppnow GR, Miley ME, Mathies RA, Liu RS, Kandori H, Shichida Y, Fukada Y, Yoshizawa T. Structure of the retinal chromophore in 7,9-dicis-rhodopsin. Biochemistry. 1990;29:8985-91.

179. Loppnow GR, Barry BA, Mathies RA. Why are blue visual pigments blue? A resonance Raman microprobe study. Proc Natl Acad Sci USA 1989;86:1515-1518.

180. Barry B, Mathies RA, Pardoen JA, Lugtenburg J. Raman microscope and quantum yield studies on the primary photochemistry of A2-visual pigments. Biophys J. 1987:52:603-10.

181. Barry B, Mathies RA. Raman microscope studies on the primary photochemistry of vertebrate visual pigments with absorption maxima from 430 to $502 \mathrm{~nm}$. Biochemistry, 1987;26:59-64. 
182. Barry B, Mathies R. Resonance Raman microscopy of rod and cone photoreceptors. J Cell Biol. 1982;94:479-82.

183. Eyring G. Curry B. Mathies R, Fransen R. Palings I, Lugtenburg J. Interpretation of the resonance Raman spectrum of bathorhodopsin based on visual pigment analogues. Biochemistry. 1980;19:2410-8.

184. Dupuis P, Harosi FI, Sandorfy C, Leclercq JM, Vocelle D. First step in vision: proton transfer or isomerization? Rev Can Biol. 1980;39:247-58.

185. Aton B, Doukas AG, Narva D, Callender RH, Dinur U, Honig B. Resonance Raman studies of the primary photochemical event in visual pigments. Biophys J. 1980:29:79-94.

186. Sulkes M. Lewis A, Marcus MA. Resonance Raman spectroscopy of squid and bovine visual pigments: the primary photochemistry in visual transduction. Biochemistry. 1978;17:4712-22.

187. Callender R. Resonance Raman studies of visual pigments. Annu Rev Biophys Bioeng. 1977:6:33-55.

188. Eyring G, Mathies R. Resonance Raman studies of bathorhodopsin: evidence for a protonated Schiff base linkage. Proc Natl Acad Sci U S A. 1979;76:33-7.

189. Deng H, Callender RH. A study of the Schiff base mode in bovine rhodopsin and bathorhodopsin. Biochemistry. 1987;26:7418-26.

190. Abdulaev NG, Nabiev IR, Efremov RG, Chumanov GD. Retinal Schiff base position relative to the surfaces of photoreceptor disk. FEBS Lett. 1987;213:113-8.

191. Deng H, Huang L, Callender R, Ebrey T. Evidence for a bound water molecule next to the retinal Schiff base in bacteriorhodopsin and rhodopsin: a resonance Raman study of the Schiff base hydrogen/deuterium exchange. Biophys J 1994;66:1129-1136.

192. Jager S, Lewis JW, Zvyaga TA, Szundi I, Sakmar TP, Kliger DS. Time-resolved spectroscopy of the early photolysis intermediates of thodopsin Schiff base counterion mutants. Biochemistry. 1997;36:1999-2009.

193. Kochendoerfer GG, Verdegem PJ, van der Hoef I. Lugtenburg J. Mathies RA. Retinal analog study of the role of steric interactions in the excited state isomerization dynamics of rhodopsin. Biochemistry. 1996;35:16230-40.

194. Loppnow GR, Mathies RA. Excited-state stucture and isomerization dynamics of the retinal chromophore in thodopsin from resonance Raman intensities. Biophys J 1988;54:35-43. (erratum Biophys J 1989:55:583).

195. Liu RS, Asato AE. The primary process of vision and the structure of bathorhodopsin: a mechanism for photoisomerization of polyenes. Proc Nat Acad Sci U S A. 1985;82:259-63.

196. Hayward G, Carlsen W. Siegman A, Stryer L. Retinal chromophore of rhodopsin photoisomerizes within picoseconds. Science. 1981;211:942-4.

197. Zhu L, Kim J, Mathies R. Picosecond time-resolved Raman system for studying photochemical reaction dynamics: application to the primary events in vision. J. Raman Spectroscopy 1999:30:777-783.

198. Bemstein PS, Yoshida MD, Katz NB, Mc Clane RW, Gellermann W. Raman detection of macular carotenoid pigments in intact human retina. Invest Ophthalmol Vis Sci 1998;39:2003-11.

199. Bernstein PS. Zhao D-Y, Ermakov IN, Mc Clane RW, Gellermann W. Resonance Raman measurement of macular pigment levels in the living human retina. ARVO abstract issue Invest Ophthalmol Vis Sci 2000;41 abstract 3185:\$600. 


\section{Chapter 3}

\section{Raman Spectroscopy Studies of Metabolic Concentrations in Aqueous Solutions and Aqueous Humor Specimens}

Applied Spectroscopy 1995.

James P. Wicksted ${ }^{1}$, Roel J. Erckens², Massoud Motamedi², and Wayne F. March²

1 Department of Physics, Center for Laser Research, Oklahoma State University. Stillwater, Oklahoma 74078

2 Department of Ophthalmology, University of Texas Medical Branch, Galveston, TX, 77555 and Biomedical Laser and Spectroscopy Program, Biomedical Engineering Center, University of Texas Medical Branch. Galveston, Texas 77555

1 Author to whom correspondence should be sent. 


\begin{abstract}
Individual concentrations of glucose, lactic acid, and urea down to $0.1 \mathrm{wt} . \%$ have been investigated in aqueous solutions using the Raman scattering technique. These measurements have been conducted using three different laser excitation wavelengths at $514.5 \mathrm{~nm}$, $632.8 \mathrm{~nm}$, and $751.8 \mathrm{~nm}$ in conjunction with a single grating spectrometer and a liquid nitrogen cooled CCD. Rabbit and Human aqueous humor specimens have also been studied using this method. In addition to a water background, the large fluorescence structure in the Raman spectra resulting from these aqueous humor specimens has been subtracted out by fitting them to a first degree polynomial. Raman peaks similar to the stretching vibrations of methylene and methyl groups associated with glucose and lactate, respectively, were observed in the human specimens within the region from $2800 \mathrm{~cm}^{-1}$ to $3050 \mathrm{~cm}^{-1}$. Similar structure was observed from the rabbit specimens. In addition, a Raman peak, indicative of the carbon-nitrogen stretching mode of urea and various amino acids, was observed at 1006 $\mathrm{cm}-1$ in all aqueous humor specimens. However, some of the Raman peaks in the rabbit specimens may have resulted from the euthanasia process.
\end{abstract}

Index Headings: Raman spectroscopy; aqueous humor; CCD detectors. 


\section{Introduction}

The eye presents a window of opportunity for noninvasive monitoring and detecting of metabolic activity and disease within the human body. Fluorescence techniques have recently been used to detect changes in the lens of the eye which are related to diabetes mellitus.(1) A photon counting method(2) to detect scattered light from the eye has been studied in an attempt to obtain information on the human body's metabolism.(3) Rabinovitch et al. have described the concept of using the aqueous humor glucose as a measure of the blood glucose concentration based on the optical rotation of the aqueous humor.(4.5)

Raman spectroscopy has seen applications in ocular lens studies(6) and for blood testing through "Raman-fluorescence" spectra.(7) In a previous article, Wang et al.(8) had used Raman scattering as a spectroscopic technique for observing metabolites, such as glucose and lactate, within aqueous solutions. The advantage of this technique is that each metabolite has a unique Raman spectrum, thus allowing several metabolites to be individually identified when mixed in water. In addition, concentrations of glucose in water, down to $0.1 \mathrm{wt}$. $\%$, could be quantitatively determined. The experimental configuration used in this work consisted of a double-grating scanning spectrometer with the Raman signal detected by a cooled photomultiplier tube. This setup required an average acquisition time of up to $5 \mathrm{~h}$ for each Raman spectrum while a laser power of $200 \mathrm{~mW}$ or more was used. Although such lengthy accumulation times and high laser powers are acceptable for in vitro studies of aqueous solutions, clearly a much shorter time period at significantly lower excitation powers are needed for the noninvasive determination of metabolic compositions within the aqueous humor of the eye.

In this article, we will discuss the Raman scattering results from various aqueous solutions containing individual metabolic molecules of glucose, lactate, and urea. In addition, in vitro measurements have been performed on human and rabbit aqueous humor specimens. The experimental setup utilizes a charge-coupled device $(C C D)$, which significantly reduces the time of acquisition of the Raman spectra and also permits the use of much lower incident laser powers than the previous study. The effects of varying laser power and acquisition times on the results will be shown. In addition, the process of subtracting water backgrounds from the data, as well as fluorescence from the aqueous humor specimens, will be discussed. Finally, the aqueous solutions have been studied using several laser wavelengths $(514.5 \mathrm{~nm}$, $632.8 \mathrm{~nm}, 751.8 \mathrm{~nm}$ ) extending our earlier work, which was conducted using only $514.5 \mathrm{~nm}$ laser light.(9)

\section{Materials and experimental methods}

Mixtures of glucose, lactic acid, and urea in HPLC water were made in order to determine the minimum concentration of these metabolites that could be observed using the Raman technique and to develop a linear relationship between the Raman integrated intensity and the concentration of the metabolite within an aqueous solution. These concentrations ranged from 0.1 to $1.0 \mathrm{wt}$ \%. The aqueous humor is mostly composed of water, ${ }^{(10)}$ with the normal concentrations of glucose, lactate, and urea within human specimens being about $0.06,0.04$, and $0.025 \mathrm{wt} \%$, respectively. Raman scattering from water therefore dominates the spec- 
trum. It should be noted that identical scans were conducted on HPLC grade, distilled, and filtered water as were performed on all the aqueous humor specimens. By utilizing the water as a reference, the concentration dependence of the Raman spectra was then examined after the water background subtraction.

The human aqueous humor samples were provided by ophthalmologists working at the Moody Laser Center in St. Mary's Hospital in Galveston, Texas. The specimens were obtained from patients during cataract surgery. The rabbit aqueous humor samples were obtained from New Zealand white rabbits within thirty minutes after euthanasia. The rabbits were injected with $5 \mathrm{cc}$ of sodium pentobarbital in the left ear. A 25 gauge needle was inserted through the cornea parallel to the plane of the iris into the anterior chamber of the eye. 0.2 to $0.3 \mathrm{cc}$ of aqueous humor were extracted from each eye.

The Raman measurements were conducted using a Spex Raman system which consisted of a Model 500M, 0.5 meter single grating spectrometer (f/4.0) and a Model Spectrum One CCD array. The spectrometer used a 1200 groove $/ \mathrm{mm}$ grating blazed at $500 \mathrm{~nm}$. The $200-$ $\mu \mathrm{m}$ slit width used resulted in a resolution of approximation $16 \mathrm{~cm}^{-1}$. The one inch CCD array consisted of 256 (row) $x 1024$ (column) pixels with each pixel $27 \mu \mathrm{m}$ in size. The CCD was cooled to $140 \mathrm{~K}$ to reduce the dark current. The CCD gain used was 16 electrons per count.

The Raman scattering experiments were performed using a right angle scattering configuration. An argon-ion laser was used to provide the $514.5 \mathrm{~nm}$ excitation wavelength with a power ranging from 5 to $25 \mathrm{~mW}$. The $632.8 \mathrm{~nm}$ laser light was obtained using a $1.82 \mathrm{~mW}$ helium-neon laser while the laser excitation at $752 \mathrm{~nm}$ was produced via an argon pumped titanium-sapphire laser. The laser light was focused to about a $100-\mu \mathrm{m}$ beam inside a capillary containing about $35 \mu \mathrm{L}$ of aqueous humor specimen. The spectrometer was controlled by an IBM computer, which also collected the signal from the CCD. Two positions of the grating were used for each of the two laser excitation wavelengths. For $514.5 \mathrm{~nm}$, the frequency ranges used were from 189 wavenumbers $\left(\mathrm{cm}^{-1}\right)$ to $1740 \mathrm{~cm}^{-1}$ and $2524 \mathrm{~cm}^{-1}$ to 3725 $\mathrm{cm}^{-1}$ while for $632.8 \mathrm{~nm}$ the ranges were from $274 \mathrm{~cm}^{-1}$ to $1286 \mathrm{~cm}^{-1}$ and $2786 \mathrm{~cm}^{-1}$ to 3489 $\mathrm{cm}^{-1}$. The lower ranges were used to study the $\mathrm{C}-\mathrm{N}$ stretching vibration of urea(11) as well as the $\mathrm{C}-\mathrm{COOH}$ peak of lactic acid.(12) The higher range was investigated in order to observe the $\mathrm{CH}_{2}$ and $\mathrm{CH}_{3}$ stretching modes of glucose and lactate respectively.(12,13) $\mathrm{A}$ holographic notch filter was used for each excitation wavelength to eliminate the intense Rayleigh scattering which occurs below $200 \mathrm{~cm}^{-1}$. The advantage of this system over the studies conducted by Wang et al.(8) is the use of the large CCD array. It has a substantially greater quantum efficiency as compared to a photomultiplier tube, as well as a much lower dark current and can simultaneously scan a much wider frequency range. This results in a much shorter acquisition time as well as a much smaller laser power for each specimen studied.

\section{Results and discussion}

The first series of experiments were conducted on separate mixtures of glucose, lactate, and urea in water in order to determine the minimum concentration for each metabolite which could be successful obtained. Several different experimental parameters were attempted for each of these mixtures to obtain those parameters yielding the best results. The removal of 
the dominant Raman modes of water has previously been discussed using a detailed subtraction process.(8) This process is illustrated in Fig. 1 for the higher Raman shifted range. Here, a $1.0 \mathrm{wt} \%$ of lactate in water is shown obtained using $12.4 \mathrm{~mW}$ of laser power at $514.5 \mathrm{~nm}$ with a scan time of $15 \mathrm{~s}$ averaged four times. A Mathcad program is used to scale the lactate in water solution (solid line) to that of pure water (dashed line) in Fig. 1(a) over the region containing the water triplet $\left(3000 \mathrm{~cm}^{-1}\right.$ to $\left.3450 \mathrm{~cm}^{-1}\right)$. This same procedure is conducted for all aqueous solutions. The region from $2800 \mathrm{~cm}^{-1}$ to $3000 \mathrm{~cm}^{-1}$ contains the $\mathrm{CH}_{3}$ symmetric and antisymmetric modes of lactate.These modes are more prominent in the water-subtracted spectrum (inset in Fig. 1a) where the lactate peaks at $2890 \mathrm{~cm}^{-1}, 2948 \mathrm{~cm}^{-1}$, and $2990 \mathrm{~cm}^{-1}$, are clearly observed.(12) Figure $1 \mathrm{~b}$ shows the water-subtracted spectrum from the lower range taken from the same sample of lactate using the same experimental conditions of laser power and acquisition time.The sloping background results from Raman scattering from the capillary. Figure $1 \mathrm{c}$ shows the same spectrum with the removal of the sloping background using a quadratic polynomial fit of this structure over the range from 500 $\mathrm{cm}^{-1}$ to $1250 \mathrm{~cm}^{-1}$. The peak at $830 \mathrm{~cm}^{-1}$ is due to the strong stretching mode between a carbon atom and the carboxylic acid group in lactate $(\mathrm{C}-\mathrm{COOH})$. Figure 2 shows two concentrations of glucose after water subtraction for the higher Raman- shifted range using $7.9 \mathrm{~mW}$ at $514.5 \mathrm{~nm}$. Figure $2 \mathrm{a}$ shows a 0.1 wt \% glucose solution taken using a $45-\mathrm{s}$ scan time averaged four times. Figure $2 \mathrm{~b}$ shows the same spectral range after using a ten-point smoothing of the data where the two peaks of the $\mathrm{CH}_{2}$ symmetric $\left(\approx 2890 \mathrm{~cm}^{-1}\right)$ and antisymmetric $\left(\approx 2950 \mathrm{~cm}^{-1}\right)$ stretching modes are more clearly resolved. Figures $2 \mathrm{c}$ and $\mathrm{d}$ show a $0.3 \mathrm{wt} \%$ of glucose with the former resulting from one 45-s scan and the latter obtained by averaging four $45-\mathrm{s}$ scans. The signal-to-noise ratio certainly improves with the averaged spectra, with the $\mathrm{CH}_{2}$ stretching modes now clearly resolved even without smoothing.

Figure 3 shows the integrated intensity over the range from 2800 to $3000 \mathrm{~cm}^{-1}$ for both glucose (Fig. 3a) and lactate (Fig. 3b) in water (after water subtraction) as the concentration of each of these two metabolites is varied. Here $\mathrm{x}$ is the metabolite concentration in wt $\%$ and $y$ is the integrated area in units of 100 counts $/ \mathrm{s} \mathrm{cm}^{-1}$. The concentration dependence vs. integrated Raman intensity follows a linear dependence, $y=m x$, with $m=2052 \pm 47$ for glucose and $m=1969 \pm 17$ for lactate. A similar linear dependence was observed for glucose.(8) During calibration of metabolites in water solutions or the characterization of an aqueous humor specimen, it is important that the same experimental conditions be maintained, such as the slit setting of the monochromator, and the temperature of the $C C D$. Best results are also observed when the same wavelength, acquisition time, and laser power are used.

Figure 4a shows a 60 -s exposure of urea, averaged eight times, taken over the lower range of the Raman scattering using the $632.8-\mathrm{nm}$ wavelength with a laser power of $1.82 \mathrm{~mW}$. The dashed curve (labeled 1) is the water Raman spectra while the solid curved (labeled 2) is the $1 \mathrm{wt} \%$ urea in water. The peak at $1003 \mathrm{~cm}^{-1}$ is the $\mathrm{C}-\mathrm{N}$ stretching vibration of urea while the smaller peak at $1158 \mathrm{~cm}^{-1}$ results from the $\mathrm{NH}_{2}$ rocking vibration.(11.14) The structure below this is due to the Raman scattering from the fused quartz capillary superimposed on the libration Raman modes of water. The spectrum below (labeled 3) results when the water and capillary Raman scattering has been successfully subtracted out so that only Raman scattering from the urea remains. Figure $4 \mathrm{~b}$ shows the same urea in water sample taken using the $514.5 \mathrm{~nm}$ wavelength with a laser power of $1.92 \mathrm{~mW}$. The time duration of this single scan was also $60 \mathrm{~s}$ and the water and capillary Raman structure have already been subtract- 

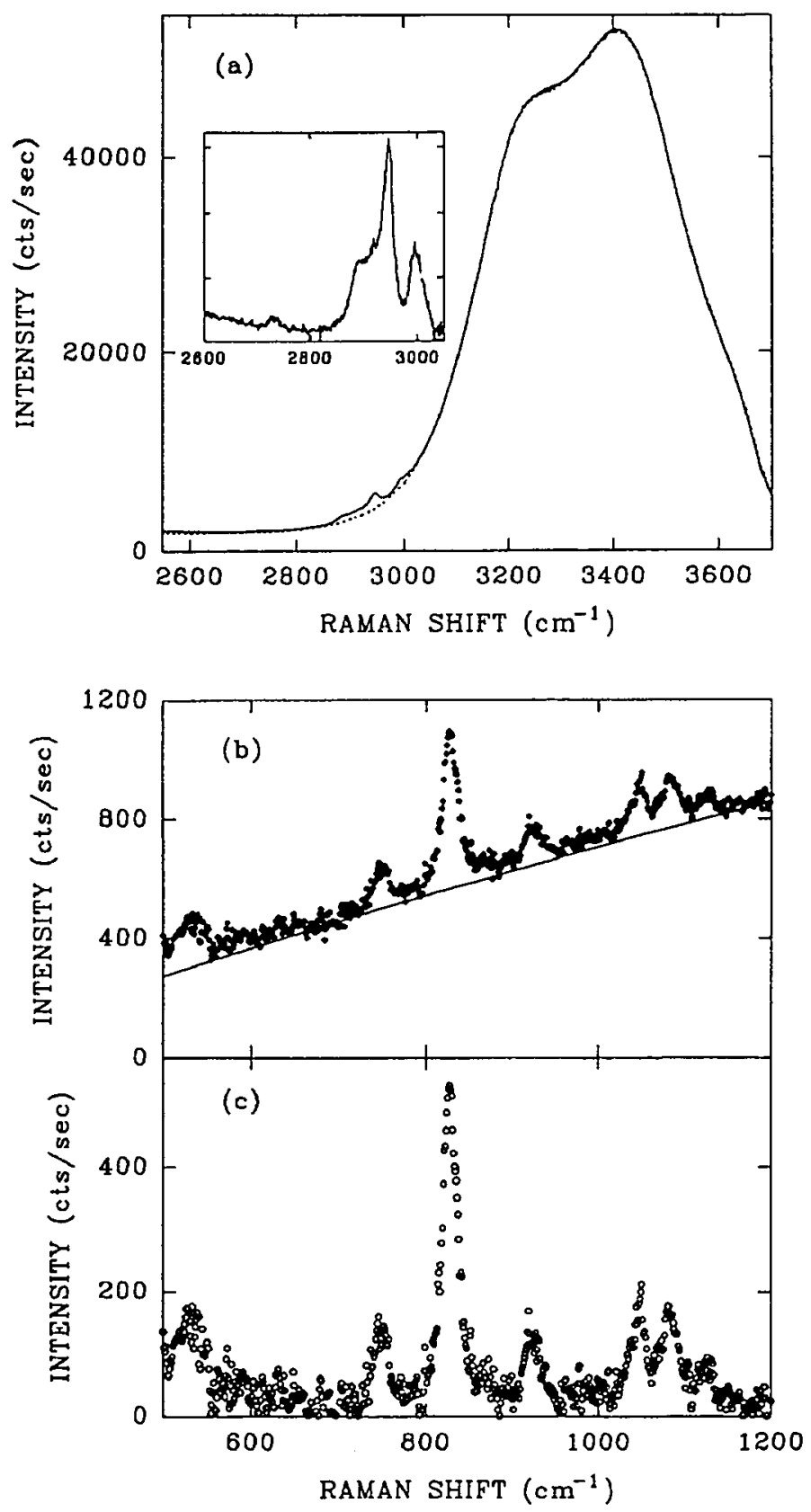

Fig. 1. (a) Raman spectra with 514.5-nm laser light of the high-frequency rang of 1.0 wt \% lactate in water is shown before and after (inset) water subtraction. The pure water spectrum in (a) is represented by the dashed curve. The low-frequency range for this same sample, after water subtraction, is also shown (b) before and (c) after an additional background is removed (see text). 


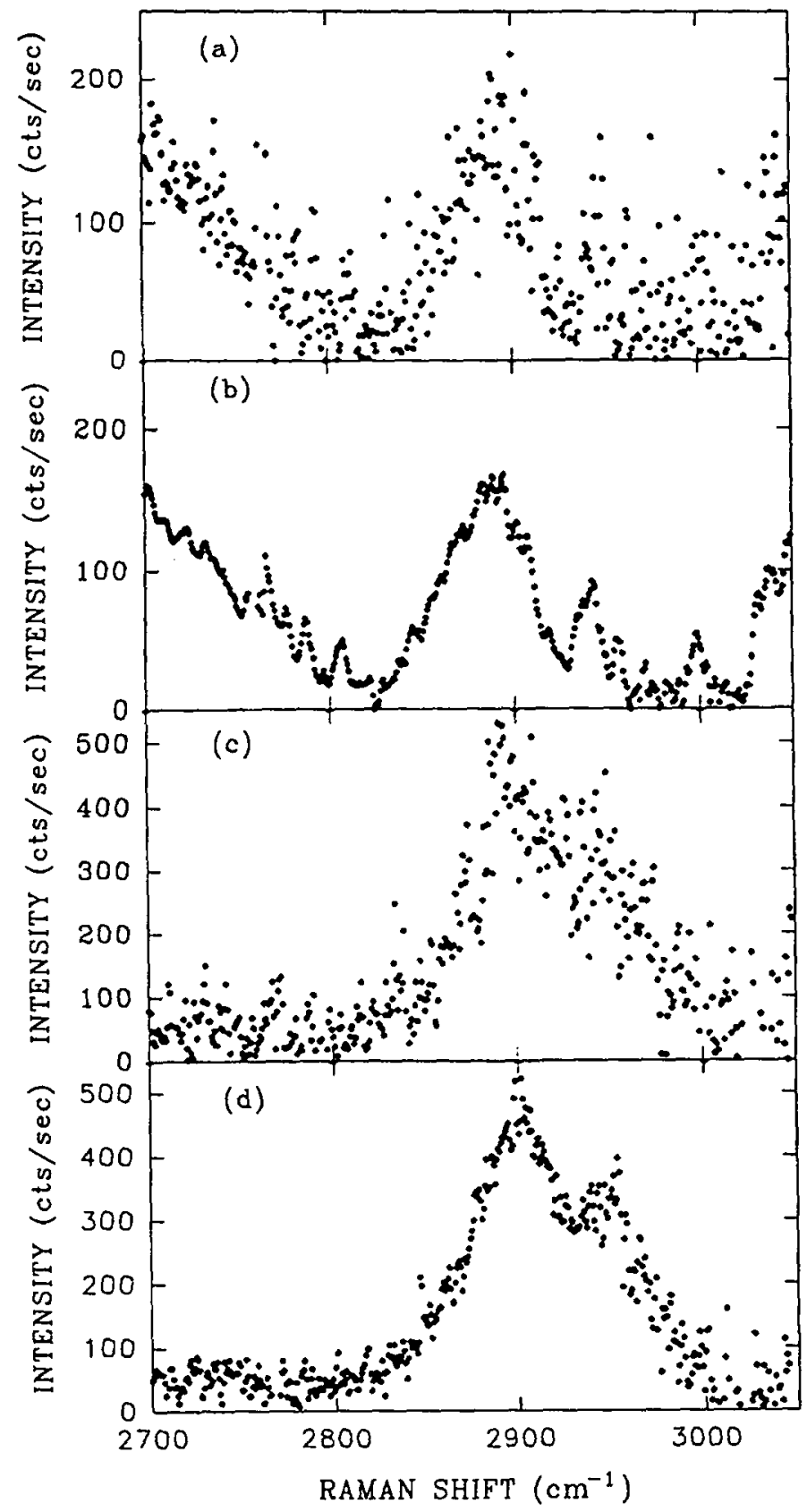

Fig. 2. Raman spectra of the high-frequency range of $0.1 \mathrm{wt} \%$ ( $a$ and $b$ ) and $0.3 \mathrm{wt} \%$ ( $c$ and $d)$ glucose in water after water subtraction with 514.5-nm laser light. The data in (b) have undergone a ten-point smoothing process as compared to (a), while the results in (d) have been averaged four times. 

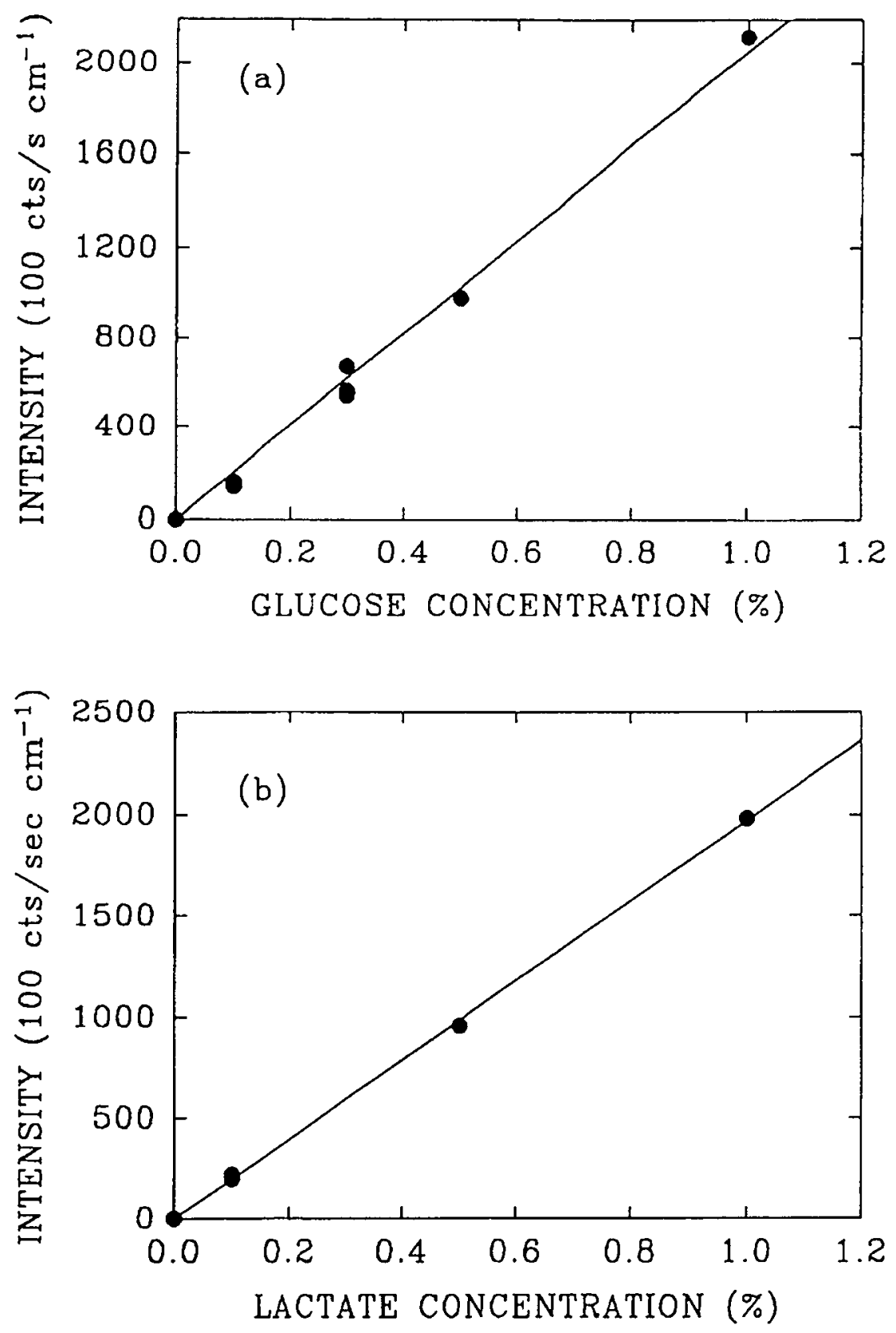

Fig. 3. Integrated Raman intensity over the range from 2800 to $3000 \mathrm{~cm}^{-1}$ for glucose (a) and lactate (b) as a function of their respective concentrations (in $w t \%$ after water subtraction). The best fit is given as $y=m x$ with $m=2052 \pm 47$ in (a) and $m=1969 \pm 17$ in (b). 

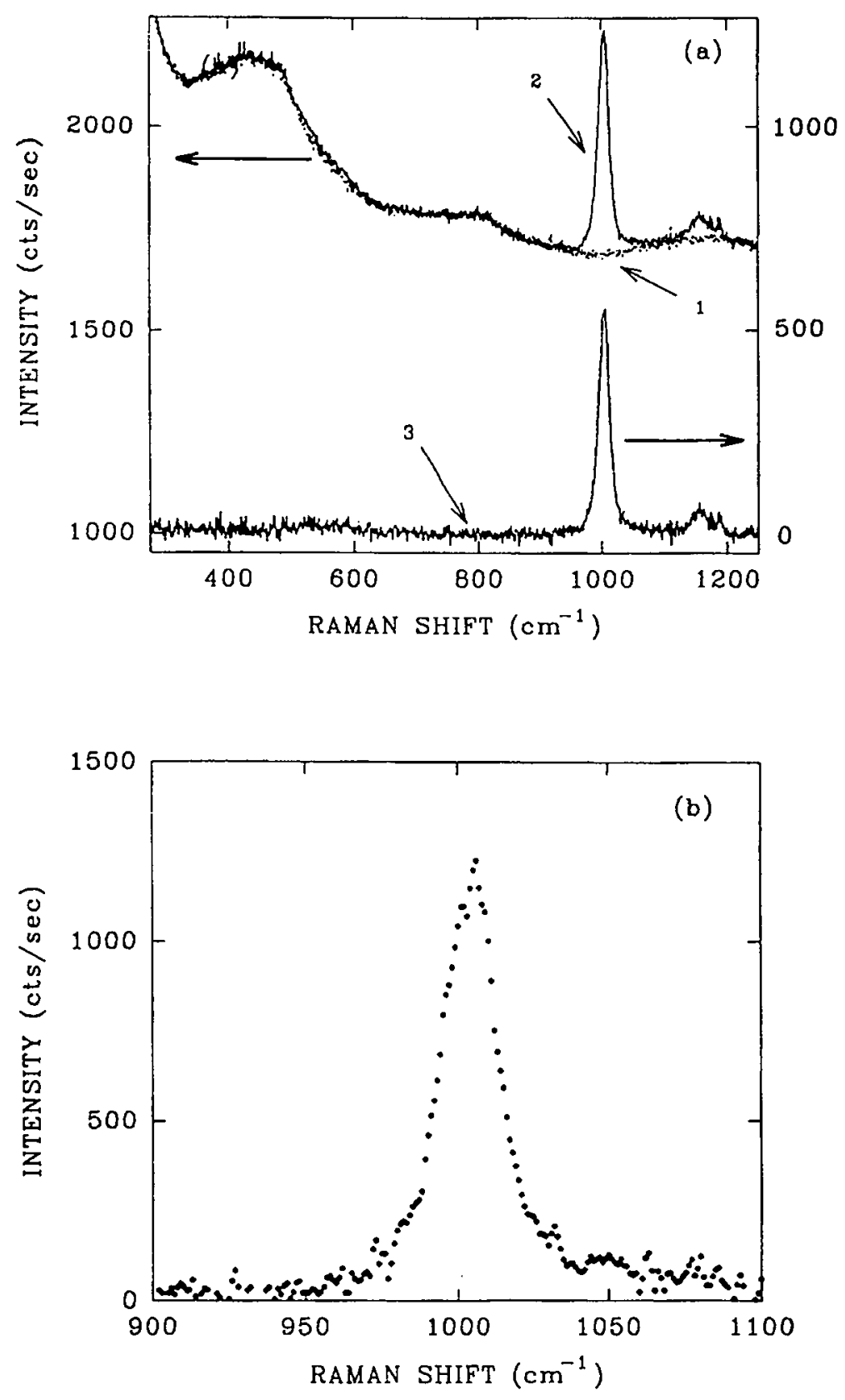

Fig. 4. (a) Raman spectra of 1 wt \% urea in water before (curve 2) and after (curve 3) water subtraction with 632.8-nm laser light. The water-capillary background is given by curve 1. The same urea concentration after water subtraction is shown in (b), where 514.5-nm laser light was used. 
ed out. What is interesting to note is that the ratio of the integrated intensity of the urea structure over the range from $950 \mathrm{~cm}^{-1}$ to $1050 \mathrm{~cm}^{-1}$ taken at these two wavelengths, normalized for the slight difference in incident laser power, scales as $\left(\lambda_{1} / \lambda_{2}\right)^{4}$ to within $2 \%$. This result is consistent with the known relationship between the integrated Raman intensity and the wavelength of the laser being used in the measurements $(I \propto \lambda-4)$.

The application of this method has been used to study both rabbit and human aqueous humor specimens. Figure 5 shows the two frequency ranges for the Raman spectra, after water subtraction, of a rabbit aqueous humor specimen. Each spectrum here resulted from averaging eight scans at $30 \mathrm{~s}$ per scan with a laser power of $20 \mathrm{~mW}$. The higher range (Fig. 5a), specifically within the region from $2800 \mathrm{~cm}^{-1}$ to $3000 \mathrm{~cm}^{-1}$, is once again showing structure characteristic of both methylene and methyl groups. Although some of this may be due to glucose and lactate, it is also possible that this structure may be resulting from sodium pentobarbital and/or amino acids which also possess $\mathrm{C}-\mathrm{H}$ stretching vibrational modes. This suggestion is based on the large intensity of the structure in the high-frequency range, as well as the positions of several prominent peaks in the lower frequency range (Fig. 5b).(9) We should note, however, that initial attempts to assay these rabbit aqueous humor specimens for sodium pentobarbital have been inconclusive. The difficulty here is due to the small volumes of aqueous humor which are available from each rabbit eye.

There are several points that should be noted about the lower frequency range of the Raman spectra from the rabbit aqueous humor specimen. First, we want to look at the large, broad fluorescence structure. It is not clear what the fluorescence mechanism within the aqueous humor is, although possible candidates are bilirubin, hemoglobin, or molecules (porphyrins) attached to proteins. Second, the prominent peak at $1006 \mathrm{~cm}^{-1}$ is most probably due to urea. In addition, the slight difference in the shift of the urea peak as compared with the measured value of $1003 \mathrm{~cm}^{-1}$ in Fig. 4 may be due to the resolution of the spectrometer or additional Raman scattering due to amino acids within the aqueous humor specimen. Finally, a large peak is also observed at about $1343 \mathrm{~cm}^{-1}$. This peak was incorrectly identified at $1380 \mathrm{~cm}^{-1}$ in our earlier paper(9). The origin of this structure is currently unknown.

The fluorescence is a major obstacle in trying to extract out the small Raman structure for the very low concentrations of metabolites within the aqueous humor. One attempt to reduce the fluorescence contribution is to use a longer laser wavelength. This approach is illustrated in Fig. 6 where two rabbit aqueous humor spectra prior to water subtraction are illustrated. The top spectrum is the same specimen used in Fig. 5a taken using the $514.5 \mathrm{~nm}$ laser light while the bottom spectrum is a different rabbit aqueous humor specimen taken using $751.8 \mathrm{~nm}$ laser light with a single acquisition of $30 \mathrm{~s}$ and an incident laser power of about $100 \mathrm{~mW}$. The large structure once again could result from sodium pentobarbital contamination, but no background subtraction is needed to clearly see the structure with the $751.8 \mathrm{~nm}$ source because of the significant reduction in the fluorescence at this longer wavelength. It should be noted that low frequency Raman data having similar structure to that obtained in Fig $5 \mathrm{~b}$ have also been obtained using the $751.8 \mathrm{~nm}$ laser light.

Another example of using this technique is shown in Fig. 7a, where the Raman spectra, after water background subtraction, of a human aqueous humor specimen is given. This spectrum was obtained using $8.1 \mathrm{~mW}$ of $514.5 \mathrm{~nm}$ laser light with a scan time of $25 \mathrm{~s}$ averaged four times. Once again, a fluorescence structure like that observed in the Raman spectra from rabbit aqueous humor specimens was observed. This fluorescence was subtracted 

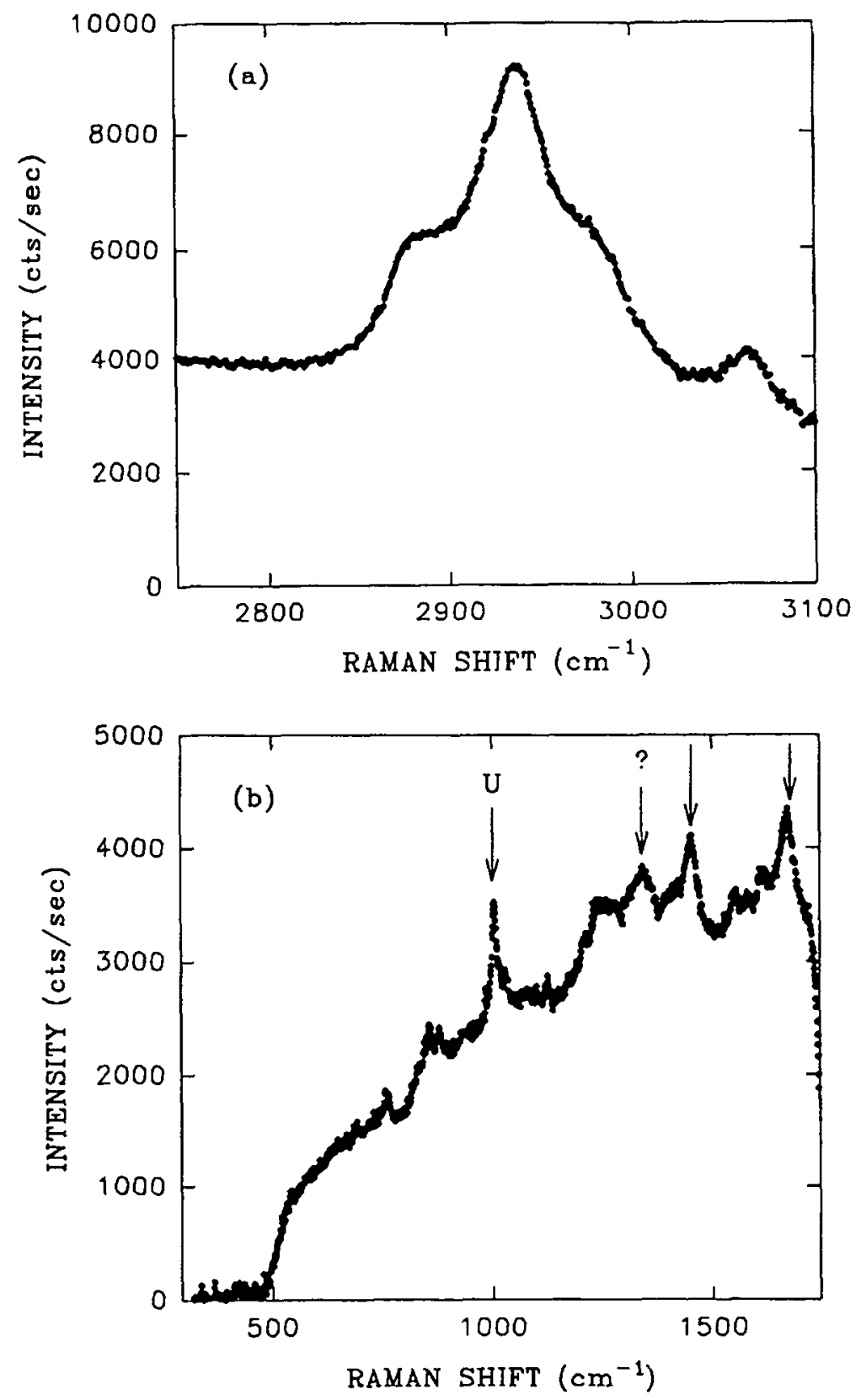

Fig. 5. Raman spectra of the rabbit aqueous humor from the (a) high-and (b) low-frequency ranges after water subtraction. The peak labeled " $U$ " at $1006 \mathrm{~cm}^{-1}$ is probably due to urea. Unlabeled peaks marked with arrows could possibly result from sodium pentobarbital and/or amino acids. The peak labeled "?" at $1343 \mathrm{~cm}^{-1}$ is of unknown origin. 

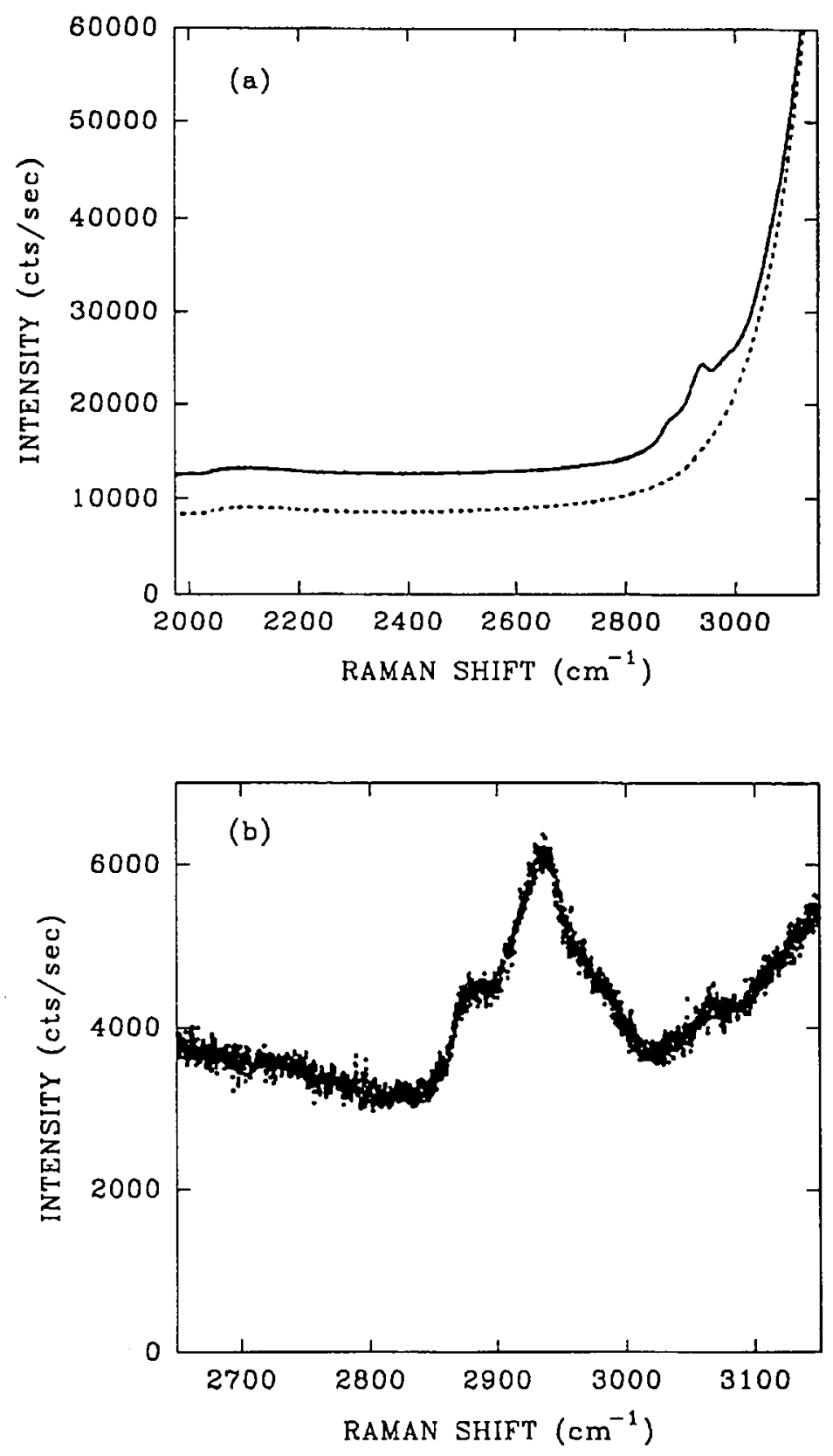

Fig. 6. Raman spectra from the high-frequency range of a rabbit aqueous humor specimen taken with (a) 514.5-nm (solid curve) and (b) 751.8-nm laser light. The spectrum in (a) is the same as that of Fig. 5a, except that no water subtraction has been made from the data. The water spectrum in (a) is indicated by the dashed curve. 


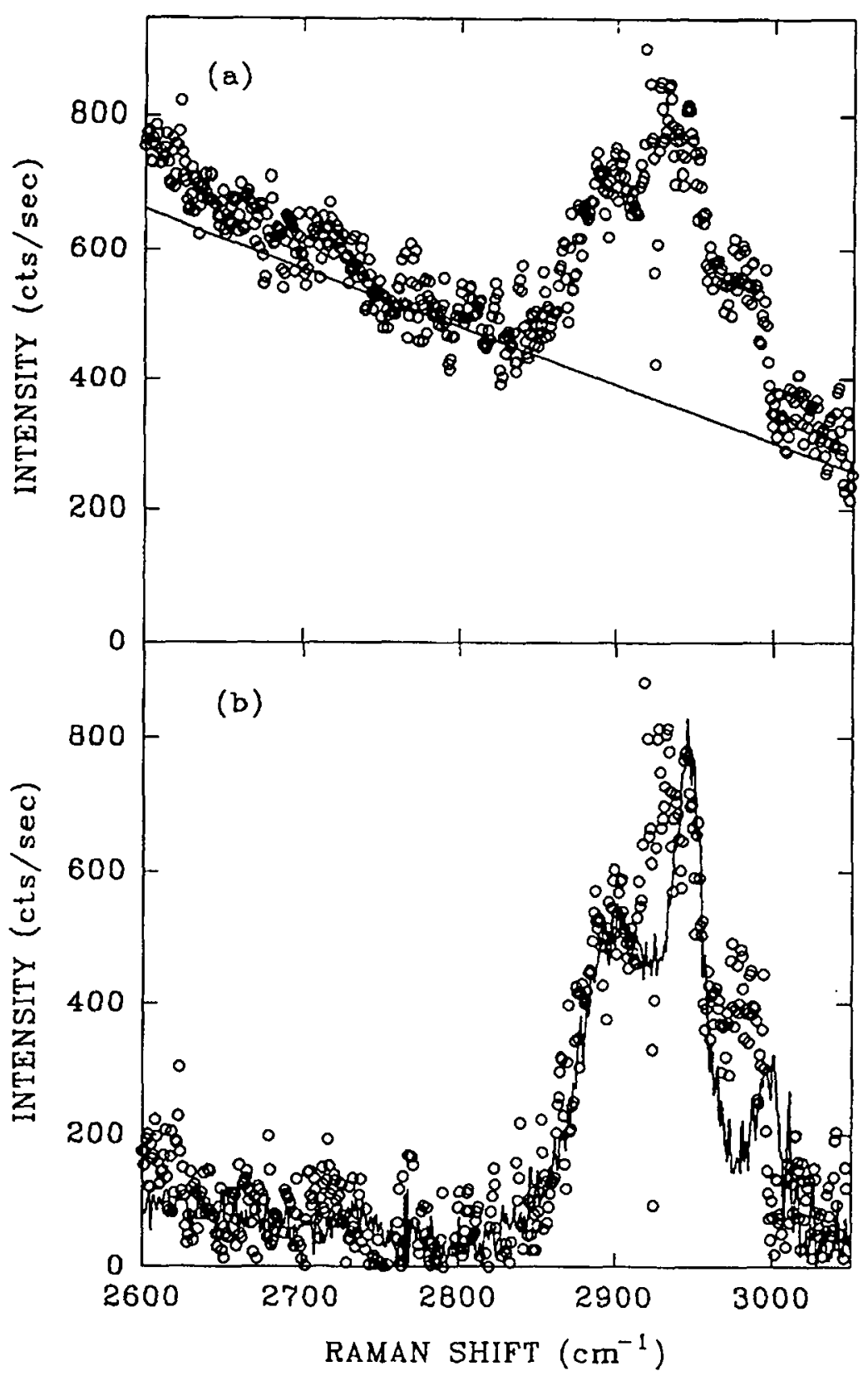

Fig. 7. (a) Human aqueous humor Raman spectra from the high-frequency range after water subtraction. The fluorescene background in (a) has been fitted to a linear polynomial (solid straight line), which is then subtracted to yield the spectra in (b) (open circles). The watersubtracted Raman spectrum of $0.2 \mathrm{wt} \%$ glucose combined with $0.2 \mathrm{wt} \%$ lactate is indicated in (b) by the solid curve. 
by fitting the background, after water subtraction, to a linear polynomial with a negative slope over the region from $2700 \mathrm{~cm}^{-1}$ to $3000 \mathrm{~cm}^{-1}$ (solid straight line in 7a), yielding the spectrum in Fig. 7b. The structure observed in this high-frequency range between $2800 \mathrm{~cm}^{-1}$ to $3000 \mathrm{~cm}^{-1}$ is indicative of a linear combination of the symmetric and antisymmetric stretching vibrations of $\mathrm{CH}_{2}$ and $\mathrm{CH}_{3}$ groups which are possibly associated with glucose and lactate, respectively. The three peaks occur at about $2893 \mathrm{~cm}^{-1}, 2939 \mathrm{~cm}^{-1}$, and $2982 \mathrm{~cm}^{-1}$. These positions should be compared to the combined structure given in Fig. $7 \mathrm{~b}$ (solid line), which is a combination of $0.2 \mathrm{wt} \%$ of glucose and $0.2 \mathrm{wt} \%$ of lactate. These data have been scaled to account for the difference in laser power and acquisition time used to obtain the human aqueous humor data. Although there is good qualitative agreement, as is illustrated in $7 \mathrm{~b}$, the concentrations used in this comparison for glucose and lactate are four times higher than are normally present within the aqueous humor. In addition, the positions of the largest structure differ with the aqueous human spectrum being about $10 \mathrm{~cm}^{-1}$ lower than the combined glucose and lactate spectrum, while the small peak at $2982 \mathrm{~cm}^{-1}$ of the aqueous humor spectrum is about $8 \mathrm{~cm}^{-1}$ lower and is higher in intensity than the combined metabolite peak at $2990 \mathrm{~cm}^{-1}$. These discrepancies may partially be accounted for by the fact that we are using lactic acid in our comparison with the human aqueous humor specimen rather than lactate ions, which have a similar structure to lactic acid over this high Raman-shifted range, but with peak positions occurring at 2935 and $2985 \mathrm{~cm}^{-1}$, in closer agreement with the aqueous humor data.(12) In addition, different water spectra were used in scaling and subtracting out the water background from the aqueous humor, glucose, and lactate data. This consideration may result in discrepancies in the intensities of the different Raman spectra, even though changes in the laser power and acquisition time were accounted for in this comparison.

\section{Conclusion}

This study has demonstrated some promising aspects of applying the laser Raman technique in order to determine metabolic concentrations within rabbit and human aqueous humor specimens. The linear dependence of glucose and lactate concentrations with the integrated Raman intensity was observed, confirming a previous investigation using a different Raman apparatus.(8) The need to biochemically assay specimens was shown when aqueous humor specimens from rabbits were studied. This approach will be continued in future measurements. The high-frequency Raman scans of the human aqueous humor specimens indicated a possible combination of methylene and methyl groups from glucose and lactate, respectively. Several laser wavelengths were used to determine how the Raman spectra of the aqueous solutions changed in both intensity and fluorescence background.

The low-frequency range of the rabbit aqueous humor specimens did show some sizeable Raman structure that may be due to sodium pentobarbital. However, it is also possible that some of the structure observed in the low frequency region may result form the various amino acid groups residing within the aqueous humor. Additional measurements are currently being conducted in order to verify the presence Raman modes resulting from amino acids and proteins within the aqueous humor.

From our earlier study, it was clear that two issues needed to be resolved concerning the use 
of Raman spectroscopy as a potential noninvasive probe of metabolites within the ocular aqueous humor. The first was the need for using lower laser powers over considerably shorter acquisition times in order to study various mixtures of glucose or lactate in water at concentrations close to those measured from aqueous humor specimens from rabbit and human eyes. This issue has been specifically addressed in this article, whereby the use of a singlegrating spectrometer and a CCD has significantly lowered both the incident laser power and the acquisition time of the Raman spectra as compared to our first measurements ${ }^{(8)}$. In addition, there is also a need to reduce the time to analyze the Raman results. Currently, Mathcad software has been used which requires considerable time to extract the metabolite contributions to the Raman spectra through the subtraction of the water structure and using polynomial fits to eliminate fluorescence structure. Recently, a multivariate calibration technique has been applied which utilizes a much shorter time of analysis while providing accurate determinations of both glucose and lactate mixtures in water solutions.(15) The combined $\mathrm{CCD}$ instrumentation with this multivariate calibration technique increases the potential for using Raman spectroscopy to noninvasively monitor aqueous humor specimens in vivo.

Current and future studies include the continuation of using the higher laser wavelength of $751.8 \mathrm{~nm}$ in order to reduce the fluorescence that has been observed from rabbit and human aqueous humor specimens. In addition, the technique of subtracting the fluorescence background that has been developed by Baraga et al.(16) on human arterial tissue using two slightly different laser excitation wavelengths will be applied to this study of aqueous humor metabolites. The development of a fiber-optic delivery and collection system using a fiberoptic Raman probe(17) to study aqueous solutions through rabbit corneas is also being conducted. We note that these studies, especially when being performed at longer wavelengths, will increase the potential for developing a compact, reliable, and inexpensive system using diode laser technology for measuring, in vivo, the metabolic activity of the human body through the eye. We also wish to point out other potential applications of this Raman scattering work such as a noninvasive means of understanding pharmokinetics associated with the eye and in vitro clinical laboratory measurements of eye tissue where the compounding effects of in vivo studies are expected to be less pronounced.

\section{Acknowledgments}

The authors wish to thank Drs. D. H. Gold and B. A. Milstein for providing the human aqueous humor specimens. The authors also thank Mr. M. J. Goetz and Dr. G. L. Cote for discussing the results of their multivariate calibration analysis prior to publication and $\mathrm{Mr}$. B. Bell for his technical assistance. This work was supported in part by a Research to Prevent Blindness Development Grant and by the Oklahoma Center for the Advancement of Science and Technology. 


\section{References}

1. J. Eppstein and S.-E. Bursell. "Non-invasive detection of diabetes mellitus", in Physiological Monitoring and Early Detection Diagnostic Methods. Proc. SPIE 1641, (1992).

2. M. Ishikawa, M. Kanebako, K. Akiyama, and T. Asakurn, Optik 84, 59 (1990).

3. M. Sawa, Jap. J. Ophthal. 34. 346 (1990).

4. B. Rabinovitch, W. F. March and R. L. Adams. Diab. Care 5, 254 (1982).

5. W. F. March, B. Rabinovitch and R. L. Adams, Diab. Care 5, 259 (1982).

6. N.-T. Yu, B. H. Jo, R. C. C. Chang and J. D. Huber, Arch. Biochem. Biophys. 160, 614 (1974).

7. K. Larsson and L. Hellgren, Experientia 30, 481(1974).

8. S. Y. Wang, C. E. Hasty, P. A. Watson, J. P. Wicksted, R. D. Stith, and W. F. March, Appl. Opt. 32, 925 (1993).

9. J. P. Wicksted, R. J. Erckens. M. Motamedi, and W. F. March, "Monitoring of Aqueous Humor Metabolites using Raman Spectroscopy," in Advances in Laser and Light Spectroscopy to Diagnose Cancer and Other Diseases, Robert R. Alfano. Editor, Proc. SPIE 2135, p. 264 (1994).

10. I. Fatt, Physiology of the eye, an introduction to the vegetative functions (Butterworths. Boston, 1978).

11. J. E. Stewan, J. Chem. Phys. 26, 248 (1957).

12. G. Cassanas, M. Morssli. E. Fabregue and L. Bardet, J. Raman Spectrosc. 22, 409, (1991).

13. P. D. Vasko, J. Blackwell and J. L. Koenig, Carbohydr. Res. 23, 407 (1972).

14. N. Wen and M. H. Brooker, J. Phys. Chem. 97, 8608 (1993).

15. M. J. Goetz, Jr.. G. L. Cote. R. J. Erckens, W. F. March, and M. Motamedi, IEEE Trans. Biomed. Eng. (in press).

16. J. J. Baraga, M. S. Feld, and R. P. Rava, Appl. Spectrosc. 46, 187 (1992).

17. C. L. Schoen. T. F. Cooney, S. K. Sharma, and D. M. Carey, Appl. Opt. 31, pp. 7707-7715, 1992. 


\section{Chapter 4}

\section{Raman Spectroscopy for Noninvasive \\ Characterization of Ocular Tissue: \\ Potential for Detection of Biological Molecules}

Journal of Raman Spectroscopy, VOL. 28, 293-299 (1997)

Roel J. Erckens', James P. Wicksted', Wayne F. March', and Massoud Motamedi'*

1 Biomedical Laser \& Spectroscopy Program and the Department of Ophthalmology \& Visual Sciences, University of Texas Medical Branch, Galveston, Texas 77555, USA.

2 Deparment of Physics, Center for Laser and Photonics Research, Oklahoma State University, Stillwater, Oklahoma 74078. USA.

* Author to whom correspondence should be addressed 
The ability of Raman spectroscopy to remotely characterize ocular tissues using a rapid fibre-optic-based system was explored in this study. An argon-ion laser operating at 514.5 $\mathrm{nm}$ and a charge-coupled-device-based Raman system were used. Raman spectra from aqueous humour specimens of rabbits and humans, as well as spectra from biological molecules obtained through fresh excised rabbit corneas, were obtained. Prominent peaks were observed which qualitatively matched the known locations from biological molecular peaks such as glucose, lactate, and urea. In the rabbit aqueous humour spectra peak positions were also observed indicative of amino acids and proteins. Raman spectra were also obtained from the rabbit lens showing peak positions indicative of amide I and III modes, sulfhydryl and $\mathrm{CH}$ stretching modes, and amino acids. This study demonstrated that it may be possible to characterize ocular tissue structures and to determine in a qualitative manner the presence of various biological molecules in aqueous humour by means of a Raman-based fibre optic instrument. 


\section{Introduction}

Noninvasive and non-destructive techniques for detecting changes in tissue composition, related to either disease or aging, and measuring different chemical substances in the human body are highly desirable. For example, measuring glucose in a noninvasive mode would be a major advantage in controlling glucose levels in diabetic patients.(1-3) In the past two decades, biomedical applications of Raman spectroscopy have been explored to develop techniques for noninvasive characterization of various tissues. Two examples of this are studies which attempt to distinguish between (i) healthy and calcified atherosclerotic regions in cardiovascular tissue(4-6) and (ii) benign and malignant human breast tissue.(7) Currently, Raman spectroscopy is in clinical use for monitoring respiratory and anaesthetic gases in the operating room. (8)

Since visible and near-infrared radiation can propagate through the cornea, aqueous humour, lens, and corpus vitreous with high transmission, the eye offers an excellent site for optical diagnosis. Thus spectroscopy-based optical diagnosis in ophthalmology could become an attractive noninvasive modality. In addition to the potential detection of biological molecules, applications of this method might include monitoring processes following photorefractive keratotomy, monitoring corneal grafts, and quality control and selection of eye bank eyes. It could also be used as a basic research tool to investigate the hydration of the cornea and lens or to investigate the pharmokinetics of drugs applied either topically or systemically to the eye. Studies using Raman spectroscopy to determine the differences between normal and cataractous lenses in various animal models are already underway.(9-11)

Recently we have conducted Raman scattering measurements from aqueous solutions, including human and rabbit aqueous humour specimens, in an attempt to determine the concentration of specific biological molecules.(12-15) These samples were contained within glass capillaries and housed within a fixed sample chamber. The goal of the current study was to investigate the potential use of Raman spectroscopy for noninvasive measurements on remote ocular tissue specimens. To achieve this goal, we integrated and optimized a fibre optic probe with a charge-coupled-detector- based Raman spectroscopy system. A series of experiments was conducted to determine the potential of this system to characterize rabbit and human aqueous humour specimens and to detect the presence of biological molecules (such as glucose, lactate, urea, amino acids). To demonstrate the capability of this instrument to remotely obtain Raman spectra of ocular tissue rapidly, (i) measurements through a rabbit cornea were conducted on aqueous solutions consisting of either glucose, lactate, or urea dissolved in water and (ii) Raman spectra from an excised rabbit lens were obtained.

\section{Materials and methods}

The fibre-optic based Raman system used in this study is illustrated in Fig. 1. A Spex single-grating spectrometer (model $500 \mathrm{M}$, Spex Industries, Edison, NJ) with a focal length of $0.5 \mathrm{~m}$ and an aperture of $f / 4$ was used. A holographic-type grating blazed at $500 \mathrm{~nm}$ with 1200 grooves $\mathrm{mm}^{-1}$ was used to disperse the light. The spectrometer was equipped with a liquid-nitrogen-cooled charge-coupled detector array (CCD) consisting of $1024 \mathrm{X} 256$ pixels with a spectral response of $400-1000 \mathrm{~nm}$. The spectrometer had a sample chamber allow- 
ing for the use of capillary tubes and collected the scattered light at an angle of $90^{\circ}$ from the incident laser beam.

$514.5 \mathrm{~nm}$ radiation from an argon laser (Model CR-4, Coherent Laser Group, Palo Alto, CA) was used for excitation. Incident laser power in the range of $25-55 \mathrm{~mW}$ was applied. Other plasma emissions from the laser were rejected by a tunable excitation filter with a grating blazed at $500 \mathrm{~nm}$ and 1200 grooves $\mathrm{mm}^{-1}$. Furthermore, a holographic notch filter at 514.5 nm (Kaiser Optical Systems, Ann Arbor, MI) was used to block Rayleigh-scattered light from the sample.

The liquid specimens used in the sample chamber of the spectrometer were placed in 100 $\mu \mathrm{L}$ borosilicate glass capillary tubes (Fisher Scientific Company, Pittsburgh, PA). First, carbon tetrachloride $\left(\mathrm{CCL}_{4}, 99.9 \%\right.$ from Aldrich Chemical Company, Milwaukee, WI) was used for aligning and optimizing the spectrometer. Next, the Raman spectra of human and rabbit aqueous humour samples were obtained. A Raman spectrum of distilled water was also obtained to account for background structures arising from the water and the capillary tube. This background subtraction has been previously described.(12)

To investigate the utility of a Raman-based fibre optic system for remote sensing, a Raman probe(16) with a two- fibre geometry (Detection Limited Technology, Waimanalo, HI) was used to perform Raman measurements at $180^{\circ}$ (Fig. 1). The excitation beam was delivered

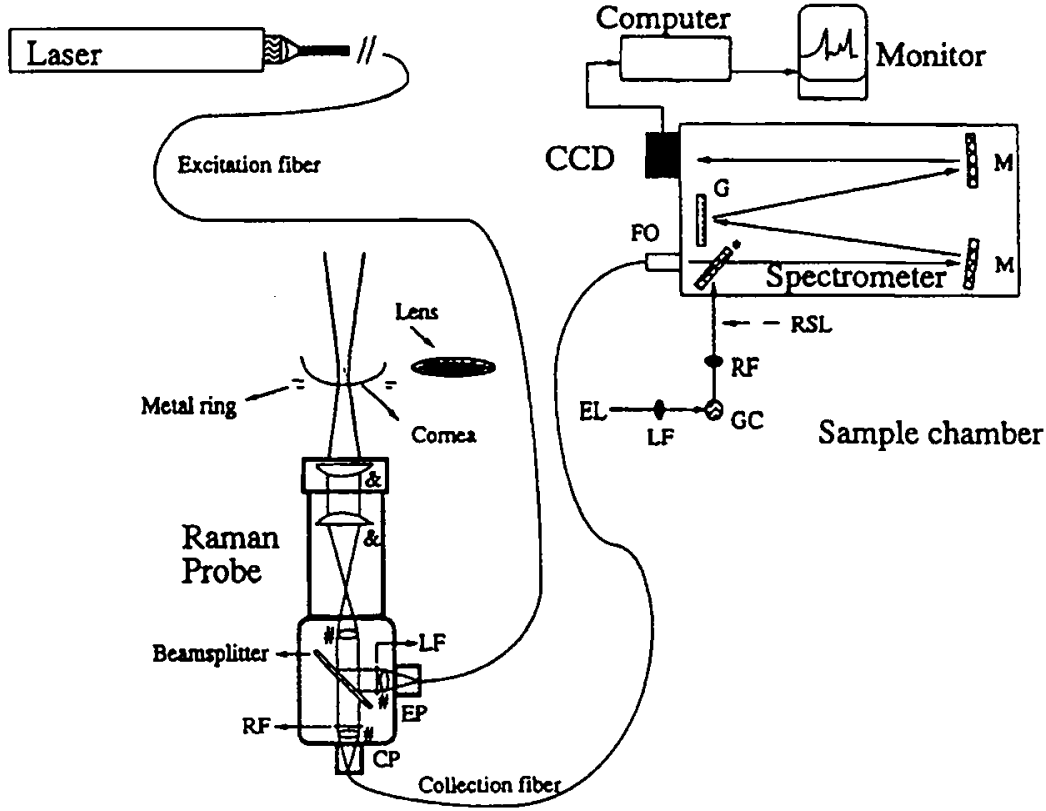

Figure 1. Raman experimental set-up for measurements in the sample chamber of the spectrometer or with the fibre optic probe: \&, plano-convex lenses; \#, collimating lenses; EL, excitation light directly from laser; $L F$, laser line filter; $G C$, glass capillary or cuvette; $R F$, Rayleigh light rejection filter; RSL, Raman-scattered light; $G$, grating; $M$, mirror; *, movable mirror; $F O$, fibre optic port; $C P$, collection port; $E P$, excitation port. 
via a $400 \mu \mathrm{m}$ diameter silica fibre (length $10 \mathrm{~m}$ ) that was connected to the excitation port of the probe. The excitation beam was then collimated and directed through a bandpass filter onto a beamsplitter. It was then focused onto the sample with a beam spot size of about 400 $\mu \mathrm{m}$. The backscattered light collected from the sample propagated through a long pass filter. It was then focused onto a $400 \mu \mathrm{m}$ diameter silica collection fibre (length $2 \mathrm{~m}$ ). The bandpass filter removed unwanted Raman and fluorescence signals from the fibre, while the longpass filter removed Rayleigh scattered light arising from the sample. The diameter of the exit/entrance lens of the probe was $22.7 \mathrm{~mm}$. The focal point of the probe was $50.8 \mathrm{~mm}$. Adjustment of the focus into the samples was possible by mounting the probe on a xyz translation stage.

Human aqueous humour specimens were obtained from six patients (four males, two females; mean age $70 \pm 5$ years) during cataract surgery and immediately placed in liquid nitrogen for transport and storage in a freezer $\left(-70^{\circ} \mathrm{C}\right)$. Rabbit aqueous humour specimens were immediately removed post mortem from six adult New Zealand white rabbits and stored at $-70^{\circ} \mathrm{C}$ as well.

To examine the capability of the proposed system to work with a more complex geometry, an excised rabbit cornea was held by a metal ring and filled with a test solution (Fig. 1). The test solutions consisted of biological molecules which were dissolved in distilled water and mixed in the following concentrations: Glucose $\mathrm{D}(+)$ in a $5 \%$ concentration (or $277.5 \mathrm{mM}$ ); Lactate in a $5 \%$ concentration (or $555 \mathrm{mM}$ ); Urea in a $5 \%$ concentration (or $825.1 \mathrm{mM}$ ). We note that these concentrations are considerably higher than the physiological levels found within the aqueous humour of a human or rabbit. However, the aim of this study was to demonstrate the capability of this Raman-based fibre optic system for remote sensing of ocular tissue.

Using the probe set-up, spectroscopic measurements were performed using comeas of three adult New Zealand white rabbits which were removed immediately after sacrificing the animals. The laser beam passed through the cornea and was focused into the aqueous solution consisting of the biological molecule mixed in water. Three corneas were used to first measure a glucose/water solution. Then, after removal of the glucose solution, the urea/water solution was added and measured. The other three corneas were filled with the lactate/water solution. The latter samples were used to avoid contamination with glucose because the Raman signals for both biological molecules overlap within the region of interest. A single Raman spectrum was obtained from each sample. A background scan, to investigate possible Raman signals arising from the cornea, was obtained from each cornea prior to introducing the solution. The background spectrum of the cornea was measured while the light was focused in the volume region where the aqueous solution was placed. Finally, Raman spectra were obtained from post mortem excised lens tissues from four New Zealand white rabbit eyes.

To test the reproducibility of the data, we first calculated the ratio of the integrated intensities of two prominent peaks (the $1010 \mathrm{~cm}^{-1}$ from the C-N stretching vibration, and the 1640 $\mathrm{cm}^{-1}$ from intramolecular bending mode of water molecules) in the human aqueous humour samples. We then computed the mean and standard deviation of these ratios for the six samples. We calculated the area under these peaks using the trapezoidal rule of integration (GRAMS/386 scientific software, Galactic Industries Corporation, Salem, NH). 


\section{Results}

Using $25 \mathrm{~mW}$ of laser power and an exposure time of $60 \mathrm{~s}$, Raman spectra from a rabbit aqueous humour specimen placed in a capillary tube were collected. The Raman spectra observed from 5 specimens exhibited similar profiles. Typical Raman spectra are shown in Fig. 2. Once the background spectra were subtracted, the Raman spectral features were mainly present in the lower spectral region $\left(700-1700 \mathrm{~cm}^{-1}\right)$, and in the higher spectral region (2600-3050 $\mathrm{cm}^{-1}$ ). We can tentatively assign peak positions to amino acids(17) (phenylalanine: $1004 \mathrm{~cm}^{-1}, 1032 \mathrm{~cm}^{-1}$; tryptophan: $\left.760 \mathrm{~cm}^{-1}, 880 \mathrm{~cm}^{-1}, 1340 \mathrm{~cm}^{-1}\right)$; the $\mathrm{CH}$ bending mode at $1450 \mathrm{~cm}^{-1}$, the CH stretching modes at $2890 \mathrm{~cm}^{-1}$ and $2945 \mathrm{~cm}^{-1}$, and the amide III mode at $1238 \mathrm{~cm}^{-1}$. Note that the water background was not completely subtracted from
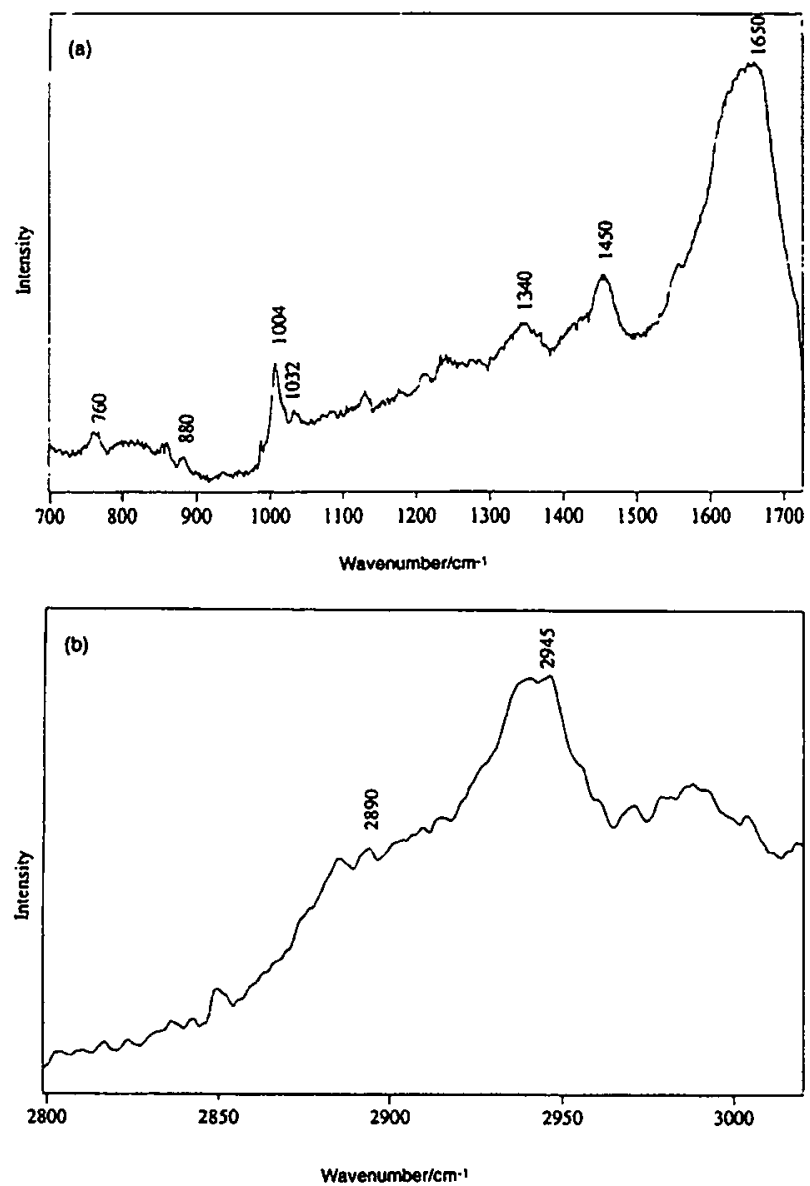

Figure 2. Raman spectra from a rabbit aqueous humour specimen for the (a) lower and $(b)$ higher spectral regions. These data was obtained while the specimen was in a capillary tube. The laser power used was $25 \mathrm{~mW}$ with an integration time of $60 \mathrm{~s}$ and the water/capillary background subtracted. 
Fig. 2(a). This is why a strong $\mathrm{OH}$ bending structure is present at $1640 \mathrm{~cm}^{-1}$.

Similar laser powers and exposure times were used to characterize human aqueous humour samples. Figure 3 shows human aqueous humour Raman spectra for two samples (denoted $\# 1$ and \#2) in the spectral region $700-1700 \mathrm{~cm}^{-1}$ and one sample in the spectral region 2600 $3100 \mathrm{~cm}^{-1}$. Figure 3(a) shows the Raman spectra before the water/capillary tube background Raman signal was subtracted, while Fig. 3(b) shows the spectrum of sample \#1 after subtraction. Raman spectroscopic signatures observed in all six specimens exhibited very similar profiles. Figure 3(c) illustrates a background-subtracted spectrum for the higher wavenumber range. The peak assignments are similar to those in Fig. 2.

To further demonstrate the reproducibility of these findings, we determined the ratio between the peak at $1010 \mathrm{~cm}^{-1}$ and the peak at $1640 \mathrm{~cm}^{-1}$ for all six human aqueous humour samples using typical spectra as shown in Fig. 3(a). Values of $0.11,0.13,0.12,0.12,0.10$, and 0.11 with a mean of 0.11 and a standard deviation of 0.01 were obtained.

Using the Raman-based fibre optic probe, Raman spectra of aqueous solutions containing known concentrations of glucose, lactate or urea placed on an excised rabbit cornea were recorded. Argon laser light of $25 \mathrm{~mW}$ was delivered through the cornea and focused into the aqueous solution. Figure 4(a) shows the spectra of the rabbit cornea before and after a glucose/water solution was added. $\mathrm{CH}_{2}$ stretching modes were observed at $2890 \mathrm{~cm}^{-1}$ and 2950 $\mathrm{cm}^{-1}$. Figure $4(\mathrm{~b})$ shows the spectra of the rabbit cornea before and after a lactate/water solution was added. Here peaks corresponding to $\mathrm{CH}_{3}$ stretching modes were observed at 2885 $\mathrm{cm}^{-1}, 2945 \mathrm{~cm}^{-1}$ and $2990 \mathrm{~cm}^{-1}$. Figure 4(c) shows the spectra of the rabbit cornea before and after adding an urea/water solution. The urea peak resulting from a $\mathrm{C}-\mathrm{N}$ stretching mode was observed at $1003 \mathrm{~cm}^{-1}$.

Figure 5 shows a spectrum obtained from a rabbit lens using the fibre optic probe with a laser power of $55 \mathrm{~mW}$ and an integration time of $2 \mathrm{~s}$. Raman peak assignments for the lens(18) tissue can be made at $3390 \mathrm{~cm}^{-1}$ (OH mode), $3063 \mathrm{~cm}^{-1}$ and $2935 \mathrm{~cm}^{-1}$ (C-H stretching modes), $2579 \mathrm{~cm}^{-1}$ (S-H stretching mode), $1670 \mathrm{~cm}^{-1}$ (Amide I mode), $1243 \mathrm{~cm}^{-1}$ (Amide III mode), $1032 \mathrm{~cm}^{-1}$ and $1004 \mathrm{~cm}^{-1}$ (Phenylalanine), and the aromatic region $880 \mathrm{~cm}^{-1}, 856 \mathrm{~cm}^{-1}, 832$ $\mathrm{cm}^{-1}$, and $760 \mathrm{~cm}^{-1}$ (tyrosine and tryptophan).

\section{Discussion}

Raman spectra obtained from the aqueous humour samples suggest that Raman signals indicative of the presence of biological molecules can be observed. These results indicate the possibility of detecting noninvasively the presence of various chemicals such as glucose, lactate, urea and amino acids in the aqueous humour. Since there is a correlation between the glucose concentration in blood plasma and the concentration in aqueous humour, (19,20) Raman spectroscopy may allow for the establishment of an accurate correlation between the amplitude of the Raman signal and the concentration of blood glucose. However, additional work is required before it can be concluded that the Raman peaks observed are due to these molecules and not some other agent having similar Raman peak positions. It should be noted that other compounds in the aqueous humour like amino acids, (21,22) which have a $\mathrm{NH}_{2}$ group and a COOH group, have Raman structures in the same region as urea and lactate. The more pronounced spectral features in the rabbit aqueous humour could be 

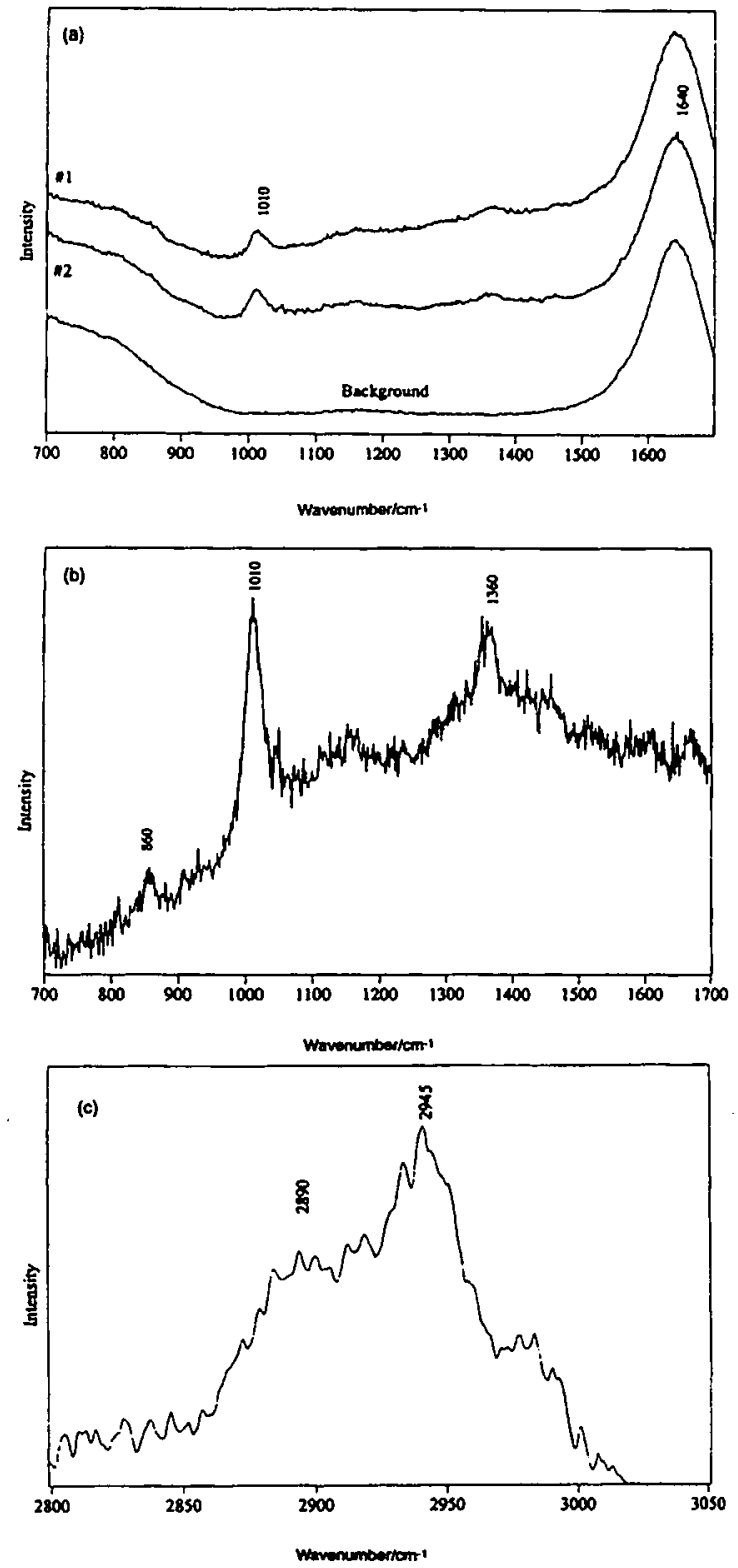

Figure 3. Raman spectra of human aqueous humour samples measured in a capillary tube using a laser power of $25 \mathrm{~mW}$ and an integration time of $60 \mathrm{s:}(\mathrm{a})$ and $(b)$ represent the scan region 700 $1700 \mathrm{~cm}^{-1}$, while (c) represents the scan region $2600-3100 \mathrm{~cm}^{-1}$ after background subtraction. In (a), the two human aqueous humour specimens are denoted by \#1 and \#2, while the background spectrum is from the capillary tube filled with distilled water. In (b), the result of the background subtraction from the aqueous humour sample \#l is shown. 

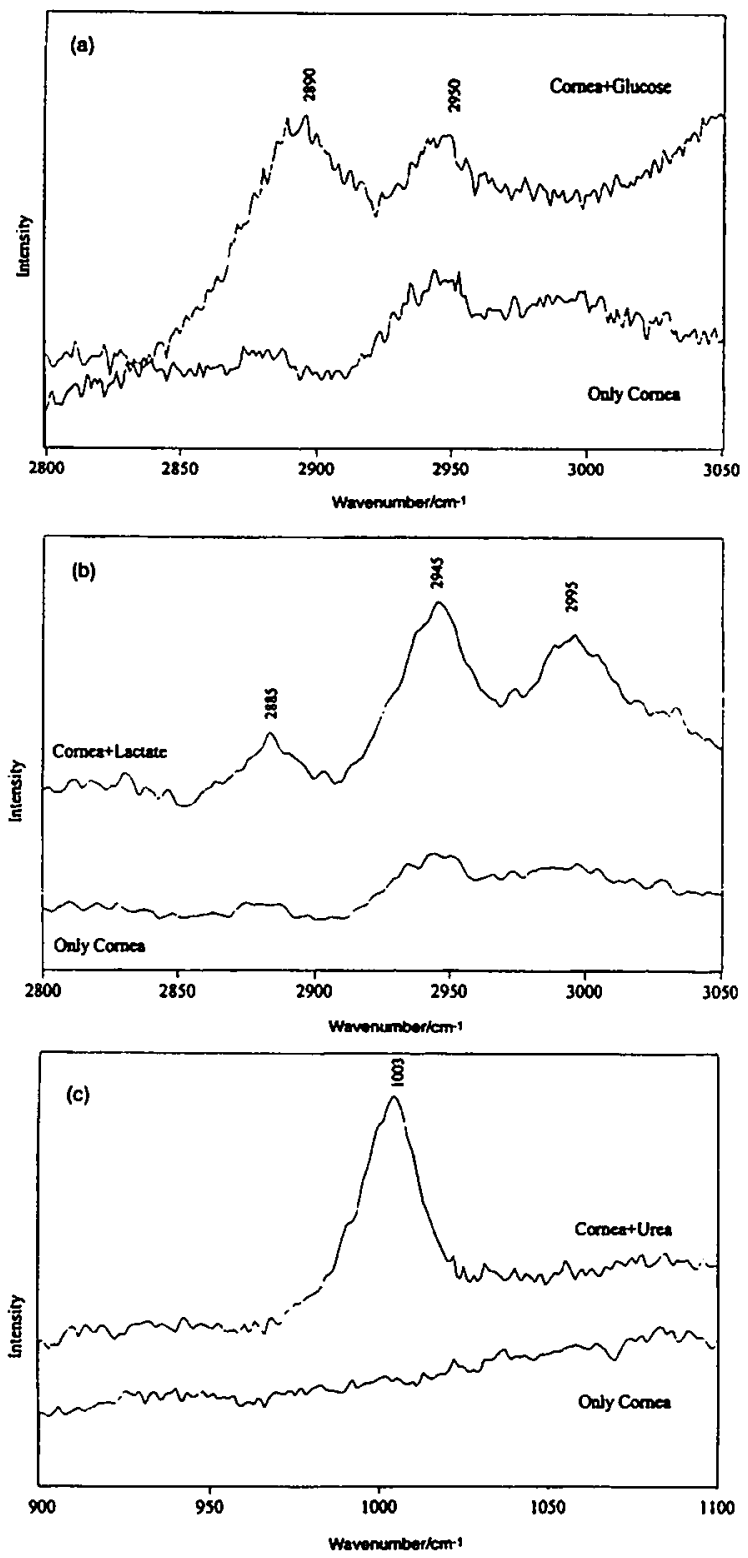

Figure 4. Raman spectra of rabbit comeas shown with added aqueous solutions of (a) 5 wt. \% glucose concentration, (b) $5 \mathrm{wt}$. \% lactate concentration and (c) $5 \mathrm{wt}$. \% urea concentration. The spectrum of the cornea is also shown. The laser power used was $25 \mathrm{~mW}$ with an exposure time of $60 \mathrm{~s}$. 


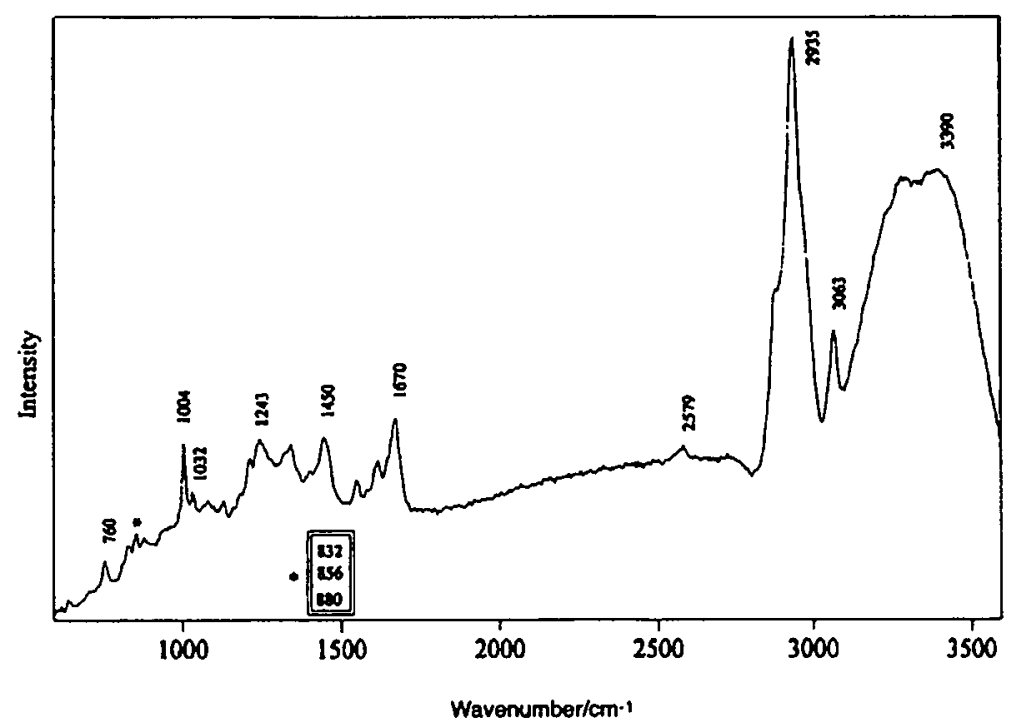

Figure 5. Spectrum of excised rabbit lens tissue. Laser power on the lens was $55 \mathrm{~mW}$ with an exposure time of $2 s$.

explained by the fact that the biological molecules in these specimens are present in higher concentrations than in the human aqueous humour. The rabbit aqueous humour concentrations are 6.7, 12.1, 6.3 and 4.1 mM for glucose, lactate, urea, and amino acids respectively. For human aqueous humour, the concentrations are 2.7-3.9, 4.5, 4.1 and $2.5 \mathrm{mM}$ for glucose, lactate, urea and amino acids respectively. Furthermore, the rabbit aqueous humour spectra were obtained post mortem, so the integrity of the eye could be compromised with possible leakage from proteins and biological molecules through the retinal barrier into the aqueous humour. The Raman activity in the human aqueous humour samples is low as would be expected. Any increased Raman activity at peak positions corresponding to amide modes or amino acid modes, could be indicative of the presence of proteins or other compounds at higher concentrations than normally present in the aqueous humour.

Finally, using the fiber optic probe configuration, it was possible to spectroscopically detect chemicals within aqueous solutions through a rabbit comea. Potential background interference and optical noise (i.e. tissue autofluorescence) that may lead to the reduction of the signal-to-noise ratio were minimized by focusing the probing argon beam within the aqueous solution. Using integration times of $2 \mathrm{~s}$ in order to obtain Raman spectra from the rabbit lens (see Fig. 5) brings this technique closer to meeting the safety standards for retinal damage while using laser light.

The next phase of this-work will incorporate a confocal capability to our Raman based fibre optic system in order to exclude the effects of adjacent tissue from the region under investigation. For example, this would allow to detect the Raman spectra from the aqueous humour without any Raman signature contribution from the cornea. 


\section{Conclusion}

This fibre-optic-based Raman system has the potential of becoming a new noninvasive diagnostic tool which, following further investigations, may be applied to the determination of biological molecules such as glucose, lactate, urea, amino acids, as well as the identification of foreign substances within the aqueous humour. It provides for remote Raman measurements and has demonstrated the capability of observing various biological molecules in aqueous solutions through rabbit corneas. This, in combination with the short exposure time $(<10 \mathrm{~s})$, a reasonable signal-to-noise ratio, and an acceptable incident laser power, justifies further pursuing potential applications of this technology in a series of in vivo animal studies.

\section{Acknowledgments}

This work has been supported in part by the Kempner Foundation, the Department of Energy (grant No. DE-FG05-91ER61226, A003), and by a Research to Prevent Blindness Development Grant. The authors would like to thank Dr. S. Trocme, M.D., Dr. D. Gold, M.D., and Dr. B. Milstein, M.D. for their assistance in collecting the aqueous humour samples. Furthermore, we wish to thank Mr. B. Bell for his technical assistance.

\section{References}

1. M. R. Robinson, R. P. Eaton, D. M. Haaland, G. W. Koepp, E. V. Thomas, B. R. Stallard, and P. L. Robinson, Clin. Chem. 38, 1618 (1992).

2. W. F. March, B. Rabinovitch, and R. L. Adam, Diab. Care 5. 259 (1982).

3. R. Tarr, The noninvasive measure of D-glucose in the ocular aqueous humor using stimulated Raman spectroscopy, Ph.D. Dissertation, Georgia Institute of Technology (1991, unpublished).

4. R. H. Clarke, E. B. Hanlon, H. Brody, and J. M. Isner, J. Raman Spectrosc. 19, 183 (1988).

5. J. J. Baraga, M. S. Feld, and R. P. Rava, Appl. Spectrosc. 46, 187 (1992).

6. J. J. Baraga, M. S. Feld, and R. P. Rava. Proc. Natl. Acad. Sci. 89. 3473 (1992).

7. R. R. Alfano, C. H. Liu, W. L. Sha, H. R. Zhu, D. L. Akins, J. Cleary, R. Prudente, and E. Cellmer, Lasers Life Sci. 4, 23 (1991).

8. D. R. Westenskow, K. W. Smith, D. L. Coleman. D. E. Gregonis. and R. A. Van Wagenen, Anesthesiology 70, 350 (1989).

9. D. DeNagel. M. Bando. N.-T. Yu, and J. J. Kuck, Invest. Ophthalmol. Vis. Sci. 29, 823 (1988).

10. A. Mizuno and Y. Ozaki, Lens Eye Tox. Res. 8, 177 (1991).

11. N.-T. Yu, J. F. R. Kuck Jr., and C. C. Askren, Curr. Eye Res. 1. 615 (1982).

12. J. P. Wicksted. R. J. Erckens, M. Motamedi, and W. F. March, Appl. Spectrosc. 49, 987 (1995).

13. R. J. Erckens, J. P. Wicksted, M. Motamedi, and W. F. March, ARVO Abstracts: Invest. Ophthalmol. Vis. Sci. 34, 936 (1993).

14. S. Y. Wang, C. E. Hasty, P. A. Watson, J. P. Wicksted, R. D. Stith, and W. F. March, Appl. Opt. 32, 925 (1993).

15. M. J. Goetz, Jr., G. L. Coté, R. Erckens, W. March, and M. Moumedi, IEEE Trans. Bio. Eng. 42, 728 (1995).

16. C. L. Schoen. T. F. Cooney, S. K. Sharma, and D. M. Carey, Appl. Opt. 31, 7707 (1992)

17. R. C. Lord and N.-T. Yu, J. Mol. Biol. 50, 509 (1970). 
18. N.-T. Yu and E. East. J. Biol. Chem. 250, 2196 (1975).

19. J. Prince, The rabbit in eye research. C.C. Thomas, Springfield, Illinois (1964).

20. S. Pohjola. Acta. Ophthal. Supplem. 88, 38 (1966).

21. E. Berman, Biochemistry of the eye, Plenum Press, New York (1991).

22. M. V. Riley, in Biochemistry of the eye, ed. by R. E. Anderson, p. 79. American Academy of Ophthalmology, San Francisco (1983). 


\section{Chapter 5}

\section{Confocal Raman spectroscopy system for noncontact scanning of ocular tissues: an in vitro study}

Optical Engineering 1997; 36(11): 3193-3199

Franciscus H. M. Jongsma1, Roel J. Erckens², James P. Wicksted³, Noel J. C. Bauer², Fred Hendrikse', Wayne F. March', Massoud Motamedi2*.

1 From the Department of Ophthalmology, Universiteit Maastricht, Maastricht. The Netherlands:

2 The Biomedical Laser \& Spectroscopy Program and the Department of Ophthalmology \& Visual Sciences, University of Texas Medical Branch, Galveston. Texas, USA;

3 The Department of Physics, Center for Laser and Photonics Research. Oklahoma State University, Stillwater, Oklahoma, USA. 


\section{Abstract}

Purpose A long-working distance fiber-optic based confocal Raman spectroscopy (CRS) system, operating in the backscatter mode, was developed for rapid non-contact characterization of ocular tissue. Materials and methods In vitro near real-time axial scanning through ocular tissue was achieved using a CCD camera and a high numerical aperture long-working distance microscope objective in a telecentric configuration. The system provides high spatial resolution $(20-150 \mu \mathrm{m})$ of transparent ocular tissues up to $13 \mathrm{~mm}$ deep into the eye in a non-contact fashion while utilizing low argon laser power and rapid scanning times (25 $\mathrm{mJ}$ ) yielding a $\mathrm{S} / \mathrm{N}$ ratio range from 30-75. To test the performance of the system for characterizing ocular tissue, Raman spectra from rabbit eyes were obtained in vitro. Results Axial scans of the cornea, the aqueous humor and the lens provided discrete and specific Raman spectra from each tissue, in both the lower and the higher wavenumber region. Characteristic Raman signals common to all tissues are the $\mathrm{OH}$-vibrations (1650 and 3100$3700 \mathrm{~cm}^{-1}$ ) and the vibrations corresponding to amino acids (Phenylalanine at $1003 \mathrm{~cm}^{-1}$, Tryptophan at 760 and $881 \mathrm{~cm}^{-1}$, and Tyrosine at $\left.646 \mathrm{~cm}^{-1}\right)$. The ocular lens can be identified by three distinct peaks (aromatic- and aliphatic $\mathrm{CH}$ stretching and $\mathrm{OH}$ bending modes) of which the aromatic $\mathrm{CH}$ stretching mode $\left(\sim 3057 \mathrm{~cm}^{-1}\right)$ is lens-specific. The cornea can be identified by the presence of two distinct peaks (aliphatic $\mathrm{CH}$ stretching and $\mathrm{OH}$ bending), and the absence of the aromatic $\mathrm{CH}$ stretching mode. The aqueous humor can be identified by the presence of the $\mathrm{OH}$ bending mode, and the lack of the both $\mathrm{CH}$ stretching modes. Conclusion A long-working distance confocal Raman spectroscopy system may offer a novel technique for the non-contact spatially resolved biochemical characterization of various tissue layers of the anterior segment of the eye. (Optical Engineering 1997; 36(11): 3193-3199). 


\section{Introduction}

The human eye provides an ideal site for the use of non-contact optical techniques for diagnostic purposes because of the transparency of its tissues to visible and near infrared light(1,2). However, most of the currently used clinical methods in ophthalmology, such as slitlamp examination of the anterior segment, fundoscopic visualization of the retina and specular microscopy of the endothelial layer of the cornea, provide no specific information on the biochemical properties of the ocular tissues ${ }^{(2)}$. This however may be of great importance since many ocular diseases involve changes in the biochemical content of the eye. For example, diabetics have an elevated level of glucose in their aqueous humor, the early onset of cataract formation involves changes in the water content of the ocular lens, and a decreased optical acuity is present as a result of increased water content and swelling of the corneal stroma in patients with decreased corneal endothelial function. Thus, a technique capable of detecting these changes in an early stage of the disease could have important diagnostic applications in the practice of ophthalmology (3.4).

In recent years many studies have explored the potential application of Raman spectroscopy for the biochemical characterization of various tissues(5-8). In particular, Raman spectroscopy has been applied to investigate various structures of the eye in vitro, demonstrating that specific Raman signals can be obtained for the cornea(9,10), the ocular lens(11-14), and the vitreous humor ${ }^{(15)}$. However, these studies have shown that the inherently weak Raman signals generally require the application of high level light doses and long scanning times in order to obtain an acceptable signal-to-noise ratio (SNR). Furthermore, the heterogeneous nature of the tissue structures can significantly contribute to the emission of sizable broad fluorescence which tends to mask most of the Raman signature. The low SNR in both situations is the main reason that in vivo ophthalmic applications of Raman spectroscopy have been limited(11).

Two recent developments could significantly improve the potential biomedical applications of Raman spectroscopy. First, confocal Raman systems have been developed in order to limit the size of the probing volume, thus minimizing the influence of autofluorescence and background noise, and effectively improving the signal-to-noise ratio(16.17). Puppels et al. recently reported on a confocal Raman microspectrometry setup, specifically optimized for the in vitro study of single cells and chromosomes, yielding high signal-to-noise ratio spectra while resolving volumes as small as $1 \mu \mathrm{m}^{3(18)}$. The second development is the application of highly sensitive CCD detectors, which has significantly contributed to the deployment of Raman spectroscopy as a powerful diagnostic tool for characterization of biological materials.

Our main objective has been to develop a Raman spectroscopy system for ophthalmic application, capable of characterizing the biochemical content of the anterior segment of the eye (cornea, aqueous humor and lens). This required the development of a flexible high-gain system capable of assessing all three tissues in a non-contact fashion while using low light dose. Furthermore, the axial depth resolution was considered to be more important in the laminated structure of the ocular tissues than the radial resolution. These considerations led to the development of a novel high-gain, long-working distance fiber-optic based confocal Raman spectroscopy (CRS) system. The system works in a backscattered mode and incorporates a high NA long working distance microscope objective lens in a telecentric config- 
uration. The system provides high spatial resolution (20-150 $\mu \mathrm{m})$ of transparent ocular tissues up to $13 \mathrm{~mm}$ deep into the eye in a non-contact fashion. The fiber-optic probe acts as the pinhole for confocal measurements and for flexible coupling with the spectrometer. The degree of confocality (and consequently the depth resolution) can be adapted to the tissue. A single grating spectrometer with a liquid nitrogen cooled CCD camera is applied for the collection of high signal-to-noise ratio spectra over a large Raman shift range. This manuscript will describe the details of this newly developed confocal Raman spectroscopy system and evaluates its performance in characterizing fresh rabbit eyes in vitro.

\section{Materials \& Methods}

A schematic of the optical components of the confocal Raman spectroscopy (CRS) system is given in Figure 1. The light source is an argon-ion laser (Coherent Radiation model CR4, Coherent Laser Group, Palo Alto, CA) emitting a linearly polarized beam at $514.5 \mathrm{~nm}$. A combination of a polarization rotator (model 310-21, Spectra Physics, Mountain View, CA) and a beam expander, consisting of a microscope objective (NA=0.66/43x, Bausch and Lomb, Rochester, NY) and a $80 \mathrm{~mm}$ achromatic lens (model 06 3200, Spindler \& Hoyer Inc., Milford, MA), is used to provide a collimated p-polarized laser beam with a diameter of $12.5 \mathrm{~mm}$, matching the back aperture of the entrance lens ("aus Jena", Planachromat LD, $\mathrm{f}=10 \mathrm{~mm}, \mathrm{NA}=0.5 / 25 \mathrm{x}$, Karl Zeiss, Jena, Germany).

To probe ocular tissue with high spatial resolution, it is important to select a microscope objective with adequate compensation with respect to the optics of the target. Most micro-

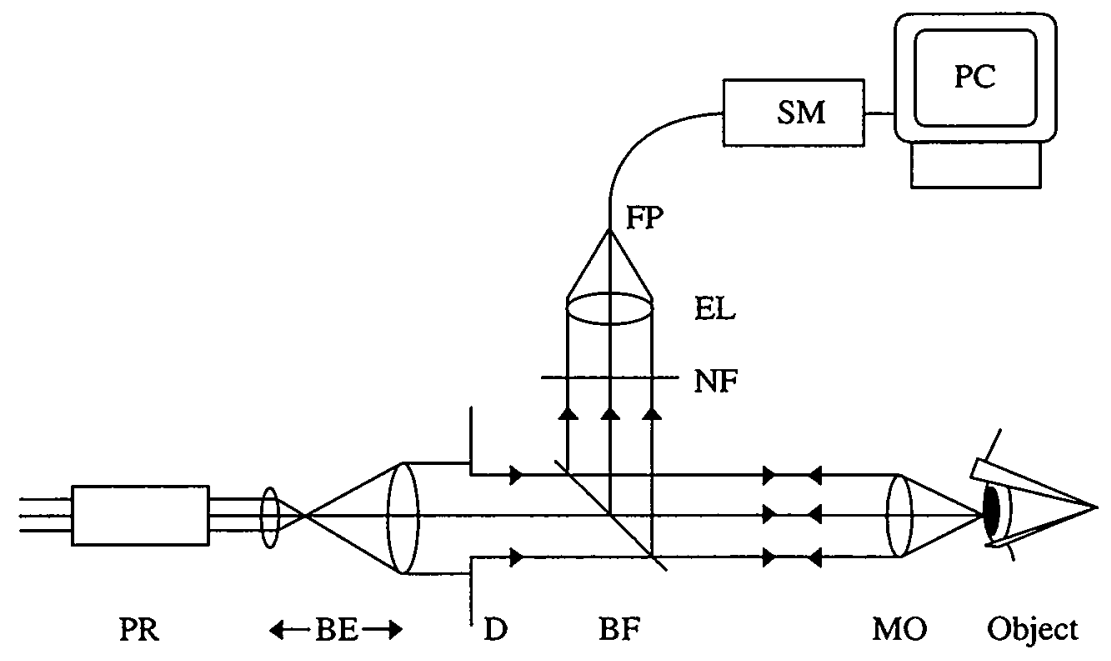

Fig. 1. Confocal Raman spectroscopy (CRS) system consisting of a polarization rotator $(P R)$, beam expander $(B E)$, diaphragm (D), bandpass filter and Raman-scattered-light reflector $(B F)$, longworking-distance microscope objective $(M O)$, notch filter $(N F)$, exit lens $(E L)$, fiber probe $(F P)$, spectrometer $(S M)$, and computer $(P C)$. See text for details. 
scope objectives are corrected for the thickness of a cover glass $(170 \mu \mathrm{m})$, and optical performance diminishes drastically in samples with greater thicknesses (for example the cornea). To ensure effective operation of the CRS system, we have chosen an objective lens as entrance lens ("aus Jena" Planchromat) that allows for correcting astigmatism that can be introduced by the optics of the target tissue (for example when probing the ocular lens through the cornea). The entrance lens used in our system is corrected for a $2 \mathrm{~mm}$ window glass ${ }^{(19)}$. If there are no optical barriers between the lens and the sample, a $2 \mathrm{~mm}$ thick correction window must be fitted onto the lens to obtain the maximum optical performance. In the integration depth measurements, with the excitation beam focused into the saline through the cuvette, the cuvette wall $(1 \mathrm{~mm})$ acts partly as the compensating window. In this case and also while probing the various ocular tissues of the in-situ rabbit eye, the correcting optical component was removed from the entrance lens.

Due to the anatomy of the eye, both non-contact detection of the Raman signal and working in a backscattered mode is desired. This is achieved when using the long working distance $(\sim 13 \mathrm{~mm})$ entrance lens, of which the secondary conjugate plane is located at infinity. This lens is employed both for focusing the laser light into the sample as well as for collecting the scattered Raman light. Since this lens is part of a telecentric configuration, we are able to obtain a free mechanical distance(20). This configuration allows us to move the entrance lens axially in order to focus the light within the sample without influencing the position and the size of the spot in the focal plane of the exit lens. Thus, the entrance lens is placed on a translation stage (model 433 with SM-50 actuator, Newport Research Co., Mountain Valley, CA) suitable for micro positioning (motion $=1 \mu \mathrm{m}$, range $51 \mathrm{~mm}$ ) and can be step wise moved along the optical axis of the Raman probe and sequentially focused in the sample of interest. A holographic beamsplitter (model 514.5NB1, Omega Optical Corporated, Brattleboro, VE) designed to work at a $45^{\circ}$ angle with respect to the laser beam serves as a bandpass filter for the incident p-polarized laser beam and also for the backscattered light, allowing most of the scattered Rayleigh light to pass while reflecting the scattered Raman shifted light sideways. The Raman shifted light is then directed through a notch filter (HNF-514-1.0, Kaiser Optical Systems Inc., Ann Arbor, MI) to minimize the effects of elastically scattered light. A camera lens ( $25 \mathrm{~mm}, \mathrm{~F}: 0.95$, Navitar, Rochester, NY) is used as the exit lens and couples the Raman scattered light into a collection fiber $(50 \mu \mathrm{m}$ core, $\mathrm{NA}=0.22$, CeramOptec, East Longmeadow, MA), which acts as the confocal pinhole and allows for flexible coupling into the spectrometer (Model 500M, Spex Industries, Edison, $\mathrm{NJ}$ ). The exit lens is diaphragmed to match both the etendue of the telecentric system and the NA (0.22) of the $50 \mu \mathrm{m}$ collecting fiber. The collecting optics on the spectrometer receives light from the fiber and focuses it with a 0.125 NA onto the $200 \mu \mathrm{m}$ entrance slit of the spectrometer. The spectrometer has a focal length of 0.5 meter and an aperture of $F: 4$. A holographic type grating blazed at $500 \mathrm{~nm}$ with 1200 grooves $/ \mathrm{mm}$, a resolution of 0.02 $\mathrm{nm}$ and a size of $110 \times 110 \mathrm{~mm}^{2}$, is used to disperse the light. The spectrometer is equipped with a liquid nitrogen cooled backthinned Charge Coupled Detector array (Spectrum One $\mathrm{LN}_{2}$, Spex Industries, Edison, NJ) consisting of $1024 \times 256$ pixels with a spectral response of $400-1000 \mathrm{~nm}$. The gain of the CCD was set at 8 electrons / count. Signal processing and presentation are performed by a computer employing DM3000S software (DM3000s ver2.50, Spex Industries, Edison, $\mathrm{NJ}$ ) interfaced with the Raman spectrometer.

In order to establish the spectral response of the entire system we have used a $100 \mathrm{~W}$ Quartz 
Tungsten halogen lamp (QTH \#6333, Oriel, Stratford, CT) at a setting of $12 \mathrm{~V}$ and a distance of $50 \mathrm{~cm}$ from the entrance lens. The lamp is positioned in its housing which contains lenses that are adjusted to get a collimated beam. The irradiance of the QTH-lamp at $50 \mathrm{~cm}$ over the spectral range from 200 to $900 \mathrm{~nm}$ was derived using curves, in power units, provided by the company. Spectra with our CRS system are obtained over a broad spectral range from 450 to $750 \mathrm{~nm}$. The spectral response of our entire system is given by: $S(I)=$ Out(I) / In(I), with Out(I) being the spectral output of our system as it measures the intensity of the light, and $\operatorname{In}(\mathrm{I})$ the real spectral irradiance of the QTH-lamp. This response is normalized for a throughput of $13.6 \%$ at $632.8 \mathrm{~nm}$, as measured with a Helium-Neon laser (NEC Co., Minato-Ku, Tokyo, Japan) in front of our entrance lens, to obtain the throughput vs. wavelength for unpolarized light.

The performance of the entire confocal system with respect to axial resolution is evaluated in a phantom medium. The phantom medium consists of a fused quartz cuvette filled with saline $(0.9 \% \mathrm{NaCl}$ Irrigation USP, Baxter, Deerfield, IL). In order to demonstrate the advantages of confocal probing, the influence of the fiber diameter on the integration depth is established, by comparing two fibers with a diameter of 50 and $400 \mu \mathrm{m}$ diameter $(400 \mu \mathrm{m}$ core, $\mathrm{NA}=0.22$, CeramOptec, East Longmeadow, MA), respectively. In both situations 25 $\mathrm{mW}$ of argon light was used and all spectra were the result of a single acquisition (1 second). The focal plane of the entrance lens was positioned outside the cuvette, and with step increments of $10 \mu \mathrm{m}$ the focus was moved towards and into the sample. After background correction, the relative Raman intensity of the $\mathrm{OH}$ bending mode at $3430 \mathrm{~cm}^{-1}$ was plotted as function of the probing depth.

The ability of the confocal setup to detect Raman spectra of various ocular tissues is examined in vitro in four normal eyes of three NZW rabbits (Ray Nichols Rabbitry, TX), less than 10 minutes following euthanasia with $100 \mathrm{mg} / \mathrm{kg}$ sodium pentobarbital (Nembutal, Abbot Labs., Chicago, IL). We used the configuration with the $50 \mu \mathrm{m}$ fiber (integration depth of $120 \mu \mathrm{m}$ in air), an incident laser power of $25 \mathrm{~mW}$, and an acquisition time of 1 second to obtain the entire spectrum. No spectral averaging was performed, unless mentioned otherwise. Axial scans with step increments of $50 \mu \mathrm{m}$ were made to obtain spatially-resolved Raman spectra of the cornea, the aqueous humor and the ocular lens. The spectral response of all three tissues is analyzed qualitatively over two Raman shift ranges; the lower region ranges from $300-1833 \mathrm{~cm}^{-1}$, and the higher region ranges from $2580-3750 \mathrm{~cm}^{-1}$. The Raman spectra were baseline corrected by subtracting the offset of the CCD-camera from the raw spectral data. The SNR for the spectral response of each tissue is calculated by dividing its maximum Raman intensity by its noise level at a region without Raman activity (usually $\left.\sim 2750 \mathrm{~cm}^{-1}\right)$.

\section{Results and Discussion}

The imperfection in the collimation of the laser beam, objective lens and the holographic beamsplitter, could introduce a considerable distortion in the probing beam size. In order to estimate the actual size of the beamwaist produced using a collimated argon laser beam and microscope objective $(25 \mathrm{x} / \mathrm{NA}=0.5)$, we inserted three pinholes with diameters of 6,12 , and $25 \mu \mathrm{m}$ in the focal plane of the entrance lens. The measurement of the transmitted laser 
power through each pinhole showed that $71 \%$ of the available laser power was present in the central $6 \mu \mathrm{m}, 21 \%$ was present in the ring between the 6 and $12 \mu \mathrm{m}$ diameters, $7 \%$ in the ring between the 12 and $25 \mu \mathrm{m}$ diameters, and the remaining $1 \%$ outside the $25 \mu \mathrm{m}$ diameter of the beam. Therefore, the diameter of the beamwaist was estimated to be $\sim 6 \mu \mathrm{m}$. Thus, when using $25 \mathrm{~mW}$ of laser power the incident irradiance is $\sim 9 \times 10^{4} \mathrm{~W} / \mathrm{cm}^{2}$.

The measured spectral response of the system is shown in Figure 2. The spectrum of the QTH lamp, the measured spectral distribution of this lamp with our CRS system, and the spectral response of the CRS system for unpolarized light are shown in Figure $2 a, b$, and c, respectively. The spectral response of our entire system depicted in Figure $2 c$, is highly influenced by the quantum efficiency of the CCD array, in the wavelength range of 400 $1000 \mathrm{~nm}$. The large drop around $515 \mathrm{~nm}$ is caused by the notch filter, which blocks any Rayleigh scattered light. The small dips around 650 and $680 \mathrm{~nm}$ are believed to be caused by the holographic beamsplitter. Since our regions of interest lie in the range of $520-630 \mathrm{~nm}$, where no significant Raman signals can be detected beyond this region, these dips are of minor importance here.

Next, the performance of the CRS system using different size collection fibers $(50 \mu \mathrm{m}$ and $400 \mu \mathrm{m}$ ) was assessed. Saline was used as a phantom because of its relatively strong Raman scattering signature and its biological compatibility. In Figure 3 the normalized Raman peak intensity of the $3430 \mathrm{~cm}^{-1}$ Raman mode of saline is plotted against the translation of the entrance lens along the optical axis for the $50 \mu \mathrm{m}$ fiber. Using this setup, a knife edge response curve for both the small $(50 \mu \mathrm{m})$ and large $(400 \mu \mathrm{m})$ collection fiber was obtained, and the depth resolution for both instances could be established. Conventionally, the depth resolution in microscopy has been defined as the full-width-at-half-maximum (FWHM) of a knife edge response curve(21.22). As described earlier by Tabaksblat et al.(23) this approach is justified in fluorescence microscopy in general but in Raman spectroscopy this approach does not suffice. In a spatial map of, for instance, a fluorescent sample, the spatial differences remain more or less visible even when they are (locally) greatly distorted by the light from out-of-focus planes. However, in Raman spectroscopic sampling where quantitative information is required, the data become unreliable with such a considerable distortion. Therefore we have arbitrarily defined the depth resolution of our system as the change in normalized Raman intensity from $10 \%$ to $90 \%$ of the maximum signal, comprising the straight part of the knife edge response curve as seen in Figure 3. Thus we determined the depth resolution of the $50 \mu \mathrm{m}$ fiber to be $118 \mu \mathrm{m}$ (Figure 3) and $920 \mu \mathrm{m}$ when using the 400 $\mu \mathrm{m}$ fiber (see also Chapter 2, Figure 8).

Some remarks about the integration depth of this confocal Raman spectroscopy system have to be made. Puppels et al. already noted that, in confocal Raman spectroscopy, the depth resolution for point like objects is controlled by a defocusing effect, in which the Raman signal decreases if the dimensions of the object are smaller than that of the light cone of the laser focus in the optical axis. This resolution however, is considerably higher than the depth resolution for planar objects (as used in this study), which mainly depends on the spatial filtering of the confocal pinhole(18). Optimizing the size of this pinhole implies a balance in blocking light from out of focus places (depth of field control) and preserving the Raman signal from the sample under investigation. Puppels et al. explain, that when a planar object is moved along the optical axis through the laser focus, the light intensity on the object decreases as is the case for the point object, but since light intensity times irradiated area 

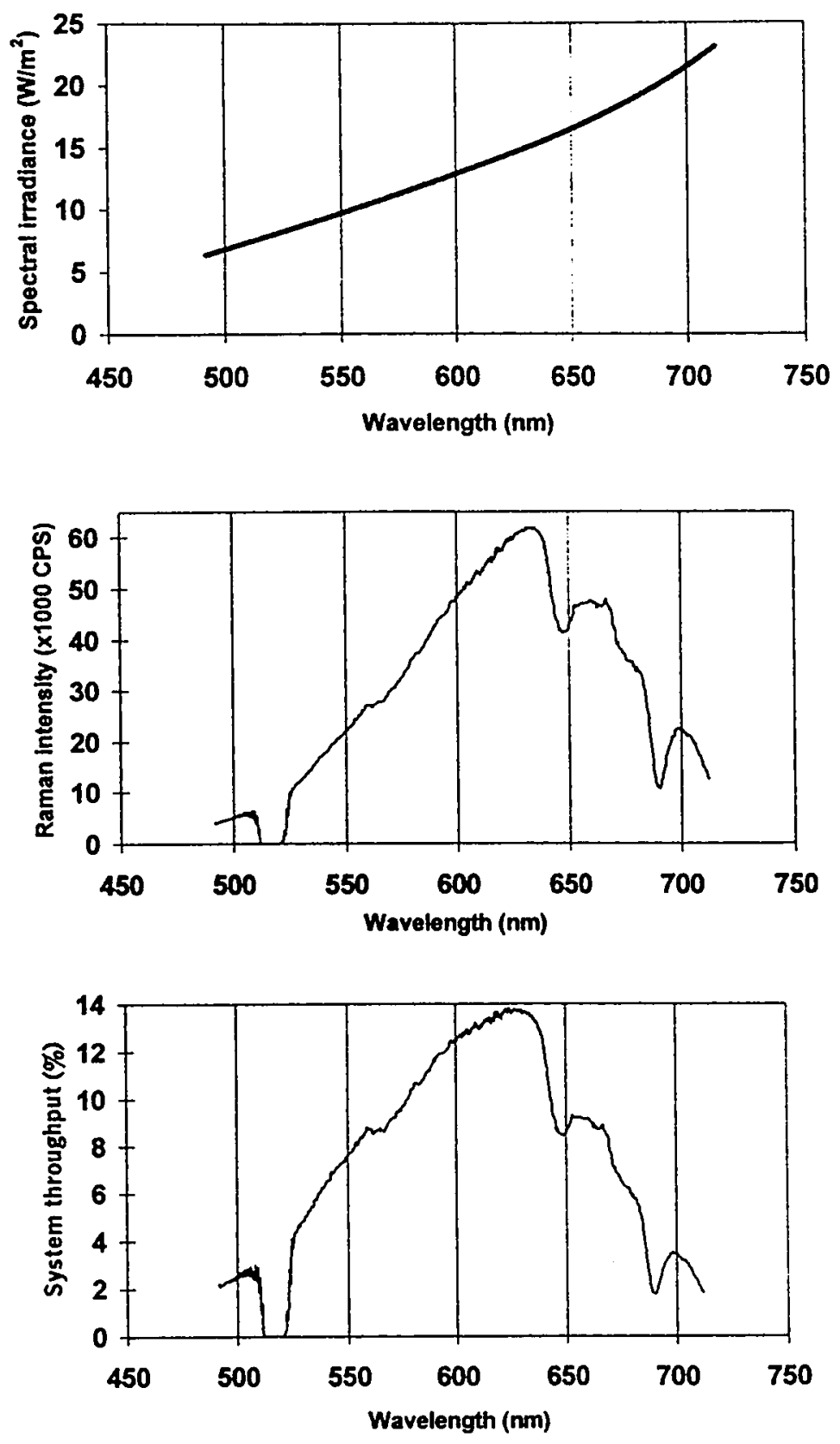

Fig. 2. Spectral response of the CRS system for unpolarized light over a range of 500 to $700 \mathrm{~nm}$, normalized for an efficiency of $13.6 \%$ at $632.8 \mathrm{~nm}$. (A) Spectral irradiance of Quartz Tungsten Halogen (QTH) lamp, $(B)$ the spectral distribution of the QTH-lamp as measured with the CRS system, (C) the resulting spectral response of the CRS system (wavelength vs, relative throughput). 


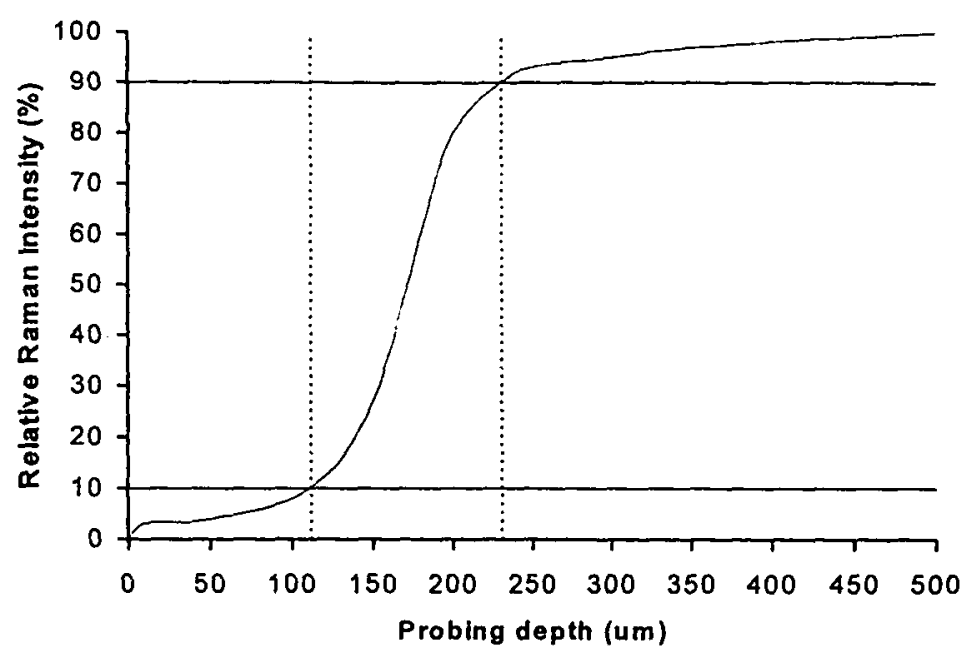

Fig. 3. Relative Raman intensity of the $\mathrm{OH}$ bending mode of water as function of the distance of the MO in relation to the sample. This resolution in depth "edge response curve" of the CRS setup yields an integration depth of $118 \mu \mathrm{m}$ with the $50 \mu \mathrm{m}$ optical fiber. The same setup with the $400 \mu \mathrm{m}$ optical fiber yielded an integration depth of $920 \mu \mathrm{m}$ (data not shown). Phantom sample was a quartz cuvette filled with $0.9 \%$ saline solution. Step increments through sample were 10 $\mu m$.

remains constant, the Raman intensity from the object will not change(18). In fact, a decrease of the Raman intensity occurs as the emitter area increases with respect to the lens diameter. This implies that the longer the working distance of the entrance lens is, the less the extent of this decrease will be. Finally, it should also be noted that due to the adaptation of the numerical aperture from the exit fiber to the spectrometer, the minimum width of the entrance slit of the spectrometer must be twice the effective diameter of the exit fiber. Consequently, it depends on the required spectral resolution if the full gain in light gathering power can be used when using larger fiber diameters. A lot of useful light was lost using the $400 \mu \mathrm{m}$ fiber at an entrance slitwidth of $200 \mu \mathrm{m}$. However, for the biochemical characterization of the ocular tissues, the $50 \mu \mathrm{m}$ optical fiber was used since it yields a relatively small integration volume and a high SNR (SNR in the cornea is 70 for a laser power of 25 $\mathrm{mW}$ and an acquisition time of 1 second). Since the combination of entrance lens ( $\mathrm{f}=10 \mathrm{~mm}$ ) and exit lens $(\mathrm{f}=25 \mathrm{~mm}$ ) provides a sample spot size of $20 \mu \mathrm{m}$ when using the $50 \mu \mathrm{m}$ fiber (depth resolution of $118 \mu \mathrm{m}$ ), this volume is considerably larger than the $1 \mu \mathrm{m}^{3}$ sample volume obtained by Puppels et al.(18), using a $63 \mathrm{x}$ water immersion objective. However, our objective is not to study single living cells or chromosomes, but to determine the positionresolved assessment of various laminated ocular tissues within the eye in a non-contact fashion.

To test the performance of our system in assessing various ocular tissues within the eye, rabbit eyes were characterized in vitro (not enucleated after euthanasia of the animal). Axial 

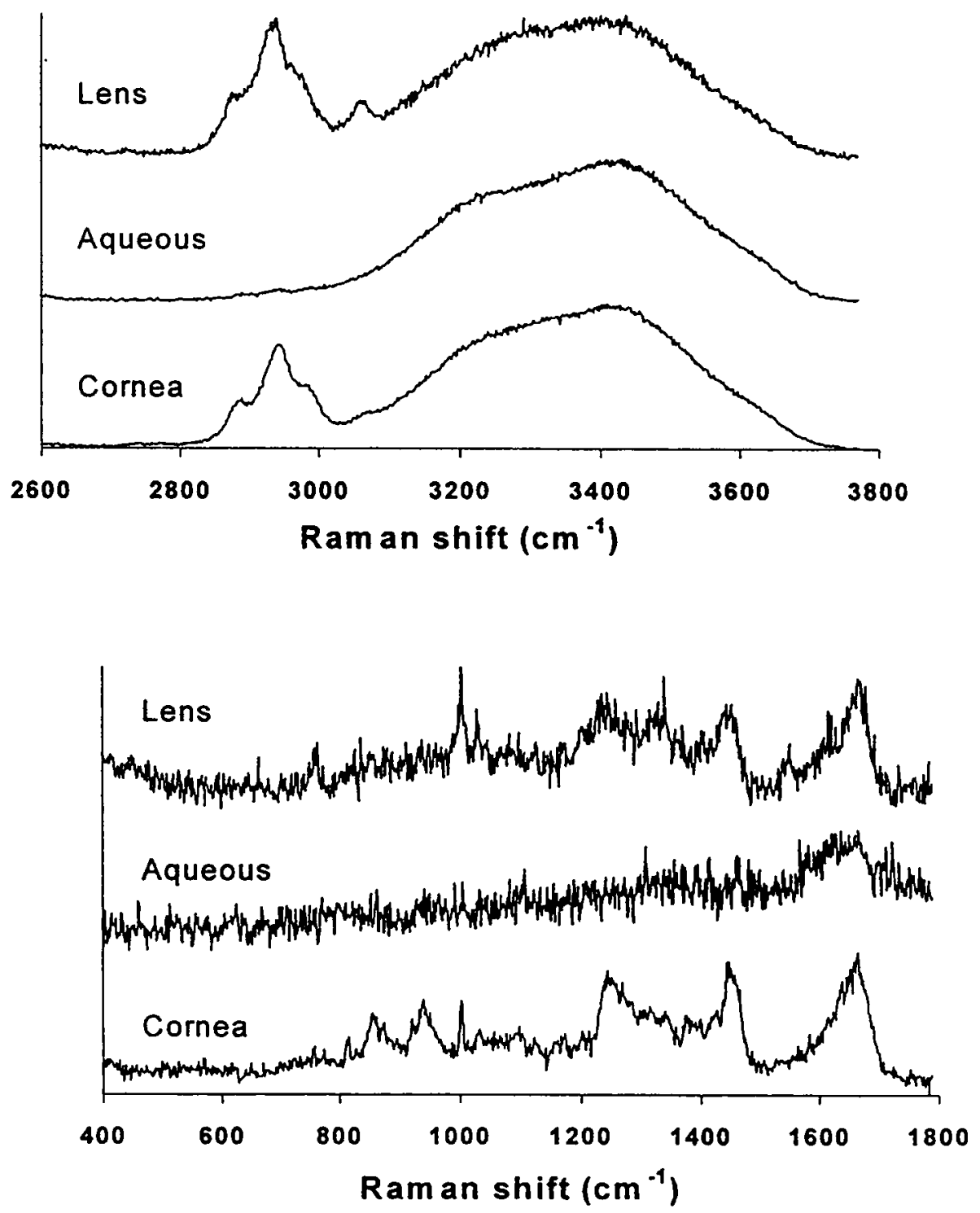

Fig. 4. Post mortem Raman spectrum of an in-situ eye of a rabbit showing typical Raman spectra for the cornea, aqueous humor, and the lens in both the lower and higher Raman shift region (see text for details). Depicted is the Raman intensity (A.U.) as function of the Raman shifted wavelength $\left(\mathrm{cm}^{-1}\right) .(A)=$ higher wavenumber region, $(B)=$ lower wavenumber region. Noteworthy is the decrease in SNR when going from the cornea through the aqueous humor to the ocular lens. SNR for the cornea, aqueous humor and lens spectra is 75, 50,30, respectively. All spectra are obtained with the $50 \mu \mathrm{m}$ optical fiber as the confocal pinhole, using $25 \mathrm{~mW}$ of sample laser power and acquisition times of 1 second per spectrum (no averaging). 
scans of the cornea, the aqueous humor and the lens provide discrete and specific Raman spectra from each tissue, in both the lower and the higher wavenumber regions, as can be seen in Figures $4 \mathrm{a}$ and $4 \mathrm{~b}$. In the lower wavenumber range (Figure 4a), we find the so-called 'signature-region'. As far as the aqueous humor is concerned, no distinct features can be seen in the lower spectral range, apart from the region around $1640 \mathrm{~cm}^{-1}$ (fundamental bending mode of $\mathrm{OH}$ ). In the cornea and the lens, we can clearly resolve several peaks. Here we identify only the most clearly resolved features of both tissues. The Amide-modes of proteins can be seen (Amide III $\sim 1225-1275 \mathrm{~cm}^{-1}$, Amide I $\sim 1640-1675 \mathrm{~cm}^{-1}$ ), and are usually used to study protein conformation, i.e. the $\alpha$-helix or $\beta$-sheet conformation of proteins(24). Furthermore, several other peaks can be identified for example those corresponding to various amino acids (Phenylalanine at $1003 \mathrm{~cm}^{-1}$, Tryptophan at 760 and $881 \mathrm{~cm}^{-1}$, Tyrosine at $\sim 646 \mathrm{~cm}^{-1}$ ) and the $\mathrm{CH}_{2} / \mathrm{CH}_{3}$-bending band at $\sim 1375-1500 \mathrm{~cm}^{-1}$ (25). The peak at $\sim 935 \mathrm{~cm}^{-1}$, which is clearly resolved in the cornea but could not be resolved in the lens, is assigned to $\mathrm{C}-\mathrm{C}$ stretching vibrations. In the higher wavenumber region from 2580 to $3750 \mathrm{~cm}^{-1}$ (Figure 4b), the strong signal of the $\mathrm{OH}$ bending mode at $~ 3100-3700 \mathrm{~cm}^{-1}$ and the $\mathrm{C}-\mathrm{H}$ stretching mode at $\sim 2850-3030 \mathrm{~cm}^{-1}$ are clearly distinguishable in both the cornea and the lens, and are mainly caused by the high amount of water and structural proteins in both tissues, respectively. The $\mathrm{OH}$ response consists of three resolved peaks(10). The $\mathrm{CH}$ signal depends on the kind of proteins or lipids found in the cornea and the lens. In the cornea mostly collagen, glycosaminoglycans and proteoglycans whereas in the lens the contribution of the lipids and crystallins can be found. In both tissues, the peak at $2886 \mathrm{~cm}^{-1}$ is most likely caused by the antisymmetric stretching mode of $\mathrm{CH}_{2}$. In both the lens and the cornea a combination of the anti-symmetric $\mathrm{CH}_{2}$ stretch $\left(2930 \mathrm{~cm}^{-1}\right)$, the symmetric stretching mode of $\mathrm{CH}_{3}(2935 \mathrm{~cm}$ 1) together with the out of plane asymmetric $\mathrm{CH}_{3}$ stretching mode $\left(\sim 2960 \mathrm{~cm}^{-1}\right)$ can be seen. The signal at $\sim 3057 \mathrm{~cm}^{-1}$ is the so-called aromatic $\mathrm{C}-\mathrm{H}$ stretching mode and, as can be seen in Figure $4 \mathrm{~b}$, its presence is rather confined to the lens. Thus, a distinction between both the cornea and the lens tissues can be made based on this finding. The aqueous humor mainly shows the spectral response of the $\mathrm{OH}$ bending mode since it almost solely consists of water, and its Raman shift position closely resembles that of pure water at $\sim 3400 \mathrm{~cm}^{-1}$. In order to investigate the reproducibility of the tissue signals, four different eyes in three different animals were compared with each other. Figure 5 shows the result in two of these samples. The spectra were obtained from about the same position within the cornea. It can be seen that the spectra show comparable profile as far as peak positions are concerned.

The Raman intensity of each specific peak depends on the concentration of the molecule(s) that cause it. Therefore, a closer analysis is always necessary to obtain either relative or absolute values for the biochemical content of a certain sample. This was investigated with regard to the phenylalanine peak intensity. The Raman intensity ratio $\mathrm{I}_{1003} / \mathrm{I}_{1640}$ represents the relative content of phenylalanine, with $I_{1003}$ being the absolute Raman intensity for Phenylalanine and $I_{1640}$ being absolute Raman intensity for the fundamental bending mode for water. For similar places in the cornea of the four different eyes this ratio was: 0.120 , $0.112,0.125,0.113$, with a mean of 0.118 and a standard deviation of 0.006 , proving high reproducibility between tissues from different subjects. 


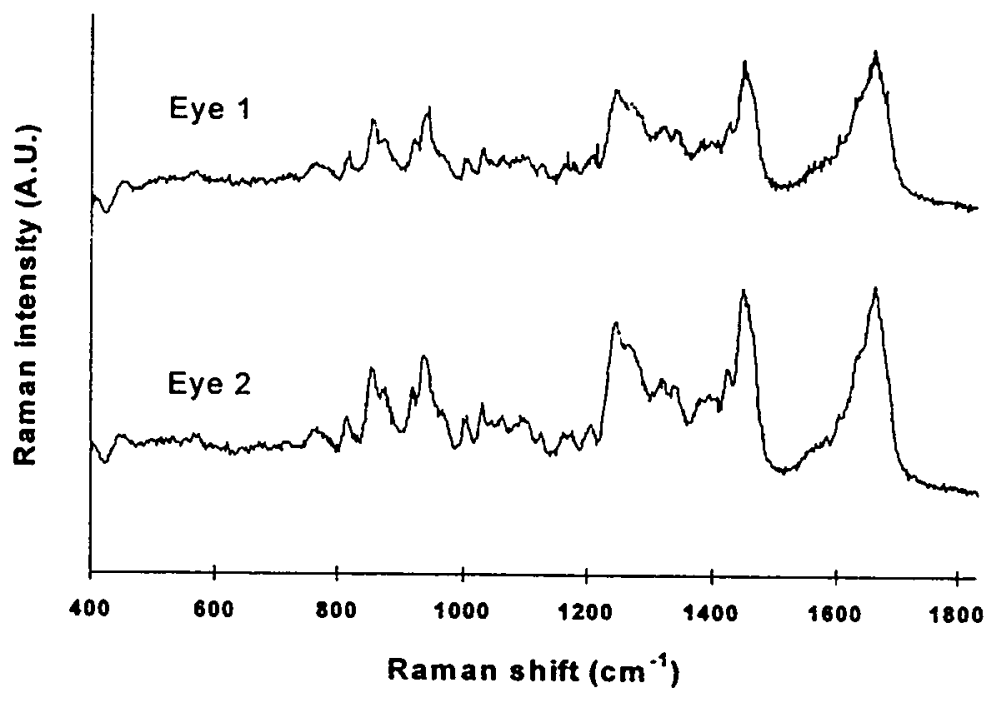

Fig. 5. Raman spectra in the lower Raman shifted region (300-1830 $\left.\mathrm{cm}^{-1}\right)$ from two eyes (left and right) of the same rabbit. Parameters: $25 \mathrm{~mW}$ sample laser power, 1 second per spectrum (no averaging, 50 um optical fiber.

\section{Conclusions}

The CRS system as presented here has several unique modalities combining the features of confocal microscopy with Raman spectroscopy. These modalities include: (1) a high NA long working-distance entrance/collection lens with correction for $2 \mathrm{~mm}$ window glass in a telecentric configuration, yielding high gain scanning properties for optical sectioning through transparent media in a non-contact fashion; (2) a rapid CCD detector array, for fast spectral acquisition of a broad Raman shift range; (3) controllable integration depth by being able to change the degree of confocality by adjusting the collection fiber diameter.

In summary, the novel confocal Raman spectroscopy system allows for the non-contact position-resolved biochemical characterization of the anterior ocular segment. However, future ophthalmic applications of this system requires determining the optimum dose of light for safe diagnosis as well as the integration of an eye tracking system which will correct for movement artifacts.

\section{Acknowledgements}

The experiments for this study were performed in the Biomedical Laser and Spectroscopy Program at UTMB, Texas and funded in part by a grant from the Department of Energy and a Research to Prevent Blindness Development Grant. 


\section{References}

1. P. Gwynne, "A new window on biomedicine with spectroscopy." Biophotonics 1, 52-59 (1994).

2. B. R. Masters, Noninvasive Diagnostic Techniques in Ophthalmology, Masters B.R. Ed., Springer-Verlag, New York, NY (1990).

3. J. Eppstein and S. E. Bursell, "Noninvasive detection of diabetes mellitus," in Proceedings of the Physiological Monitoring and Early Detection Diagnostic Methods Symposium, SPIE 1641, (1992).

4. J.A. van Best and P.H. van Gessel, "Autofluorescence and light scatter in the human lens as measured by a fluorophotometer," Experimental Eye Research 49, $511-3$ (1989).

5. C.J. Frank, R.L. McCreery and D.C. Redd, "Raman spectroscopy of normal and diseased human breast tissues," Analytical Chemistry 67, 777-83 (1995).

6. A. Mizuno. T. Hayashi, K.Tashibu, S. Maraishi, K. Kawauchi and Y. Ozaki, "Near-infrared FT-Raman spectra of the rat brain tissues," Neuroscience Letters 141, 47-52 (1992).

7. R.H. Clarke, J.M. Isner, T. Gauthier, K. Nakagawa, F. Cerio, E. Hanlon, E. Gaffney, E. Rouse. and S. DeJesus. "Spectroscopic characterization of cardiovascular tissue," Lasers in Surgery \& Medicine 8, 45-59 (1988).

8. M. Pezolet. M. Pigeon-Gosselin, R. Savoie and J.P. Caille, "Laser Raman investigation of intact single muscle fibers. On the state of water in muscle tissue." Biochimica et Biophysica Acta 544, 394-406 (1978).

9. S. C. Goheen, L.J. Lis, and J.W. Kauffman, "Raman spectroscopy of intact feline comeal collagen," Biochimica et Biophysica Acta 536, 197-204 (1978).

10. D.S.W. Siew, G.M. Clover, R.P. Cooney, P.M. Wiggins, "Micro-Raman spectroscopic study of organ cultured corneae." J. Raman Spec. 26, 3-8 (1995).

11. N-T. Yu, J.F.R. Kuck.Jr. and C.C. Askren, "Laser Raman spectroscopy of the lens in situ, measured in an anesthetized mbbit," Current Eye Research 1. 615-618 (1982).

12. N-T. Yu, M.Bando, J.F.R. Kuck, "Fluorescence/Raman intensity ratio for monitoring the pathologic state of human lens," Investigative Ophthalmology \& Visual Science 26, 97-101 (1985).

13. A.C.C. Bot, A. Huizinga, F.H.M. de Mul, G.F.J.M. Vrensen, J. Greve. "Raman microspectroscopy of fixed rabbit and human lenses and lens slices: new potentialities," Experimental Eye Research 49, 161-169 (1989).

14. S. Dai, S. Qi. L. Zhang, C. Bai. T. Ni, X. Deng, "Laser Raman spectrometry study on experimental galactose-induced cataract. Yen Ko Hsueh Pao (Eye Science) 11(3), 143-6 (1995).

15. J. Sebag, S. Nie, K. Reiser, M.A. Charles, and N-T Yu. "Raman spectroscopy of human vitreous in proliferative diabetic retinopathy." Investigative Ophthalmology \& Visual Science 35, 2976-80 (1994).

16. J. Pawley, Fundamental Limits in Confocal Microscopy. Handbook of biological confocal microscopy, J.B. Pawley, Ed., pp. 15-26. Plenum Press. New York and London (1990).

17. G.J. Brakenhoff, P. Blom, and P. Barends, "Confocal scanning light microscopy with high aperture immersion lenses," J. of Microsc. 117, 219-32 (1979).

18. G.J. Puppels, W. Collier, J.H.F. Olminkthof. C. Otto, F.M.M. de Mul, and J. Greve, "Description and performance of a highly sensitive confocal Raman microspectrometer," J. Raman Spec. 22, 217-25 (1991).

19. H. Beyer, H. Riesenberg, Handbuch der Mikroskopie, VEB Verlag Technik, Berlin 1988).

20. D. W. Slaaf, R. Alweijnse, and H. Wayland, "Use of telescopic imaging in intravital microscopy: a simple solution for conventional microscopes." International Journal of Microcirculation: Clinical and Experimental 1, 121-134 (1982).

21. H. T. M. van der Voort, G. J. Brakenhoff, and G. C. A. M. Janssen, "Determination of the 3-dimensional optical properties of a confocal scanning lasermicroscope," Optik 70, 48-53 (1988).

22. R. W. Wijnaendts van Resandt, H. J. B. Marsman, R. Kaplan, J. Davoust, E. H. K. Stelzer, and R. Stricker, "Optical fluorescence in three dimensions: microtomoscopy," J. Microsc. 138, 29-34 (1985). 
23. R. Tabaksblat, R. J. Meier, and B. J. Kip. "Confocal Raman Microspectroscopy: theory and application to thin polymer samples," Appl. Spec. 46. 60-8 (1992).

24. I. Siebinga, G.F.J.M Vrensen, K. Otto, G.J. Puppels, F.F.M. de Mul, J. Greve. "Ageing and changes in protein conformation in the human lens: a Raman microspectroscopic study," Exp. Eye Res. 54, 759-67 (1992).

25. A. Mizuno and Y. Ozaki, "Aging and cataractous process of the lens detected by laser Raman spectroscopy," Lens \& Eye Tox. Res. 8(2-3), 177-187 (1997). 


\section{Chapter 6}

\section{Drug induced corneal hydration changes monitored in vivo by noninvasive confocal Raman spectroscopy}

In press in J Raman Spectr.

Roel J. Erckens M.D., 1,2 Franciscus H. M. Jongsma Ph.D.,' James P. Wicksted Ph.D., ${ }^{3}$ Fred Hendrikse M.D. Ph.D., 1 Wayne F. March M.D.,2 Massoud Motamedi Ph.D.2

1 Department of Ophthalmology. Academic Hospital Maastricht. Maastricht. The Netherlands.

2 Laser \& Spectroscopy Program of the Center for Biomedical Engineering and the Department of Ophthalmology and Visual Sciences, University of Texas Medical Branch Galveston, Galveston, Texas 77555-0456, U.S.A.

3 Department of Physics. Center for Laser and Photonics Research. Oklahoma State University,Stillwater. Oklahoma 740783072, U.S.A.

Reprint requests: Roel J Erckens, M.D. Department of Ophthalmology, Academic Hospital Maastricht, P. Debeyelaan 25, Postbus 5800, 6202 AZ Maastricht. The Netherlands.

Phone: 31-0621203131, Fax: 31-433875343. E-mail: re@wxs.nl 


\begin{abstract}
It is well established that the state of corneal hydration plays a crucial role in maintaining optimal vision. Therefore, any knowledge that can be obtained noninvasively about the status of corneal hydration could be of significant clinical value. A novel confocal Raman spectroscopic technique is used to monitor noninvasively drug induced hydration changes in the rabbit cornea. The spectroscopic technique enables one to monitor the changes in water content of the cornea while the confocal probing reduces interference of signals from adjacent tissues and allows for measurement of corneal hydration at various depths. The corneal hydration is altered by applying a dehydrating agent (Muro $128^{\circ}$ ) topically on the cornea. To determine the corneal hydration status, the ratio $\mathrm{OH} / \mathrm{CH}$ between the Raman intensity of the water $\mathrm{OH}$ mode at $3390 \mathrm{~cm}^{-1}$ to the protein $\mathrm{CH}$ stretching mode at $2945 \mathrm{~cm}^{-1}$ is calculated. In the middle of the corneal stroma after $10 \mathrm{~min}$., Muro $128^{\circ}$ treated corneas show an average decrease of about $30 \%$ in the $\mathrm{OH} / \mathrm{CH}$ ratio $(1.27 \pm 0.13)$ as compared to the untreated corneas $(1.76 \pm 0.09)$. In this in vivo model it is possible to monitor the hydration status of the living cornea using the Raman spectroscopic technique.
\end{abstract}




\section{Introduction}

The ability of the cornea to transmit visible light is partially dependent on the hydration status of this tissue. Hydration of the stroma is mainly controlled by the corneal endothelium, a single layer of cells located in the posterior region of the cornea(1-2). Changes in the stromal hydration may interfere with corneal transparency since the optimal spacing of the stromal collagen fibrils can be altered(3). Corneal edema, which is an excessive accumulation of fluid in the stroma(4-5), can be associated with the use of topical applied drugs for treating eye diseases (e.g. glaucoma(6)), degenerative Fuchs dystrophy, inflammatory-like acute graft rejection, herpes zoster keratouveitis, severe iritis, and elevated intraocular pressure. Hydration changes can also be associated with type I diabetes(7) and contact-lens-induced stromal acidosis. In addition, it has been shown that the excimer laser ablation rate of the cornea during photorefractive keratectomy (PRK) is influenced by the degree of tissue hydration ${ }^{(8-9)}$. If the tissue is dehydrated, the laser ablation rate tends to increase. Also, central islands could form after photoablation due to nonuniform fluid distribution in the comea during the procedure(10). Hence information on the degree of corneal hydration and the spatial distribution of water in the cornea, as well as the kinetic of water removal or accumulation in the cornea, is important when making a diagnosis or treating the cornea.

Investigations on assessment of corneal hydration have been mainly limited to use of indirect measurement techniques such as biomicroscopy of the endothelial cells in which the cell count is indicative of tissue vitality(11-12). In recent years, applications of various invasive and noninvasive techniques have been proposed to assess comeal hydration. These techniques include optical sectioning(13) and mechanical sectioning ${ }^{(14)}$ of the cornea, refractive index measurements(3), nuclear magnetic resonance spectroscopy(15-16), laser induced breakdown spectroscopy(17) and differential scanning calorimetry(18). However, it remains a challenge to obtain information on the hydration status of the living cornea without compromising tissue integrity, while still achieving a clinically acceptable spatial resolution and rapid probing time.

Raman spectroscopy provides information on molecular vibrations induced by monochromatic probing light. The inelastically scattered monochromatic light reveals a spectral shift that is specific for the molecular structure. Such a spectral shift is recorded as a Raman spectrum.

In ophthalmology Raman spectroscopy has been mainly used to characterize cataractinduced changes in the biochemical structure of the lens ${ }^{(19-21)}$ and the retina(22-25). We have used this technique to perform in vitro studies on human and rabbit aqueous humor specimens in an attempt to identify biological molecules such as glucose and lactate(26-27).

In order to apply this technique in vivo, we developed a confocal Raman scattering system(28). Essential to the confocal design is that the probing sample volume is limited, preventing detection of interference signals and fluorescence from adjacent ocular tissues. This sample volume is adjustable by changing the diameter of the collection fiber, which acts as the pinhole in the system. Furthermore, the probing beam diverges rapidly thereby improving retinal safety. This system was used to characterize the axial corneal hydration gradient in both in vitro and in vivo rabbit eyes(29) and compared the estimation of the total corneal hydration with conventional measurements, such as pachymetry and lyophilization, while also a Raman spectrum of a human cornea in a blind patient was obtained(30). Recently, the 
molecular structure of an implanted intraocular lens was determined in a patient using a retina safe laser power excitation setting(31).

In this study we investigate the use of in vivo confocal Raman spectroscopy to observe drug induced changes in the corneal hydration from rabbit eyes. The hydration changes in the cornea are achieved by topical application of the dehydrating agent Sodium Chloride 5\% (Muro $128^{\circ}$ ointment, Bausch \& Lomb ${ }^{\otimes}$ ). Muro $128^{\circ}$ is commercially available and is used in the clinic to treat corneal edema. The Raman spectral features analyzed are present in the higher spectral region (2600-3400 $\left.\mathrm{cm}^{-1}\right)$. These Raman vibrational modes are indicative of the presence of proteins ( $\mathrm{CH}_{2}$ and $\mathrm{CH}_{3}$ stretching modes) with a peak at about $2945 \mathrm{~cm}^{-1}$ and water $(\mathrm{OH})$ with the higher and broader structure with the highest peak occurring around $3390 \mathrm{~cm}^{-1}$. Changes in hydration are inferred by determining the changes in the ratio of the Raman intensity of the water to that of protein structure $(\mathrm{OH} / \mathrm{CH})$.

\section{Materials}

The Raman measurements obtained in this study utilize a confocal Raman spectroscopy system that has been recently described in the literature(28). The major instruments of this system are an argon laser (CR-4 model from Coherent Laser Group, Palo Alto, CA) as the excitation source $(514.5 \mathrm{~nm}$ ), a planachromat (25x NA=0.5; ausJena, Jena, Germany) microscope objective with a $12 \mathrm{~mm}$ working distance that focuses the incident laser light and collects the backscattered Raman signal, and a single grating spectrometer (SPEX 500M; Spex Industries, Edison, $\mathrm{NJ}$ ) with a nitrogen cooled Charge Coupled Detector array (CCD) consisting of 1024 X 256 pixels. A 50 micrometer diameter fiber ( $2 \mathrm{~m}$ long) that transmits the Raman signal to the spectrometer and acts as the pinhole in the confocal configuration, providing a 130 micrometer integration depth in the cornea. All Raman spectra are obtained using an incident laser power of $25 \mathrm{~mW}$ and an exposure time of one second.

The in vivo mammalian stroma has a hydration of about $3.5 \mathrm{~g}$ water/g dry material(32). There is an apparent direct correlation between corneal stroma thickness and its hydration(33) and it has been found that the dry tissue densities of the cornea among some mammalians [e.g. rat, rabbit, man] are the same(34). The rabbit cornea has been used as a viable model in eye research(35). The thickness of the rabbit cornea is about 400 micrometers. The rabbit cornea epithelium is about 50 micrometers thick (5-7 cells deep). However, $90 \%$ of the cornea thickness is made up by the stroma and contains the lamellae with the collagen fibrils.

Raman spectroscopic data from ocular tissues of six adult male New Zealand White rabbits (average weight $4 \mathrm{~kg}$ ) are obtained. The animals are treated according to the ARVO Resolution on the use of Animals in Research. The animals are anesthetized using $25 \mathrm{mg} / \mathrm{ml}$ Ketamine and $10 \mathrm{mg} / \mathrm{ml}$ Xylazine, repeated every 30 minutes. The animal head is fixated in a stereotactical holder with height control. The spectrometer, probe and stereotactical holder are mounted on an optical bench (Newport, Irvine, CA).

As a reference point, we position the confocal optical probe in the superficial region of the cornea in such a way that a Raman signal of about 80 counts above baseline is obtained at the $\mathrm{CH}$ peak position (i.e. $2945 \mathrm{~cm}^{-1}$ ). The Raman vibrational mode of this $\mathrm{CH}$ peak is probably generated by the Type I collagen in the corneal stroma, since this type of collagen makes up to $90 \%$ of the cornea(32). In this position the probing light is placed about 67 
micrometers into the cornea. This depth was estimated assuming an index of refraction of the cornea of about 1.375 and including the fact that in air the integration depth of the confocal system is about 100 micrometers. This is the starting position and from here on the probe is moved axially with increments of 50 micrometers into the stroma (Figure 1). The $400 \mu \mathrm{m}$ thick cornea of the rabbit has been scanned with increments of $50 \mu \mathrm{m}$, yielding about $30 \%$ of the signal height of the linear part from the knife edge response curve(36) of the normalized Raman signal.

The degree of corneal hydration at baseline is assessed by moving the probing volume stepwise through the cornea thus defining the air/cornea interface (bottom spectrum in Figures $1 \mathrm{~A} \& \mathrm{~B}$ ) and the cornea/aqueous humor interface (top spectrum shown in Figure $1 \mathrm{~A}$, the sixth spectrum from bottom in Figure 1 B). Next, about $1 \mathrm{ml}$ of the Muro $128^{\circledR}$ sterile oph-

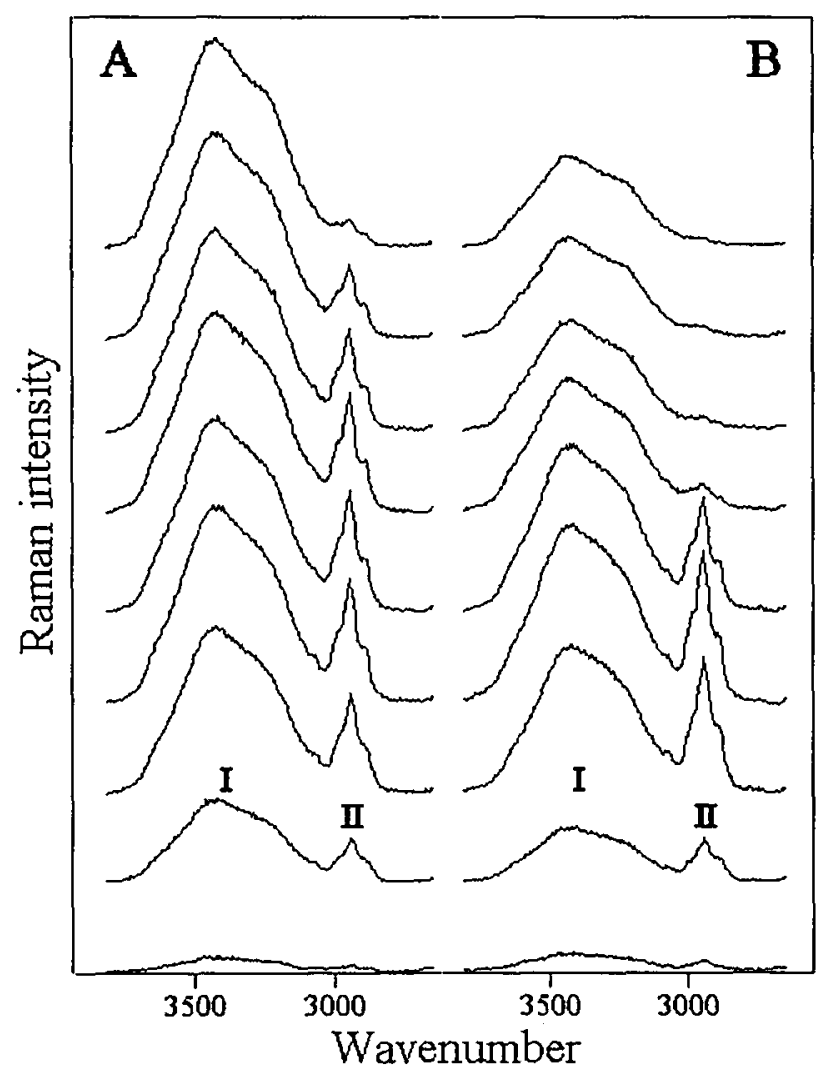

Figure 1. Axial scan consisting of nine Raman spectra spaced 50 micrometers apart.

normal cornea, (b) Muro treated cornea. The bottom scan represents the air-cornea interface.

Each subsequent scan shows a 50 micrometer displacement of the focussing point of the probe into the cornea. The top scan in the figure shows a scan of the cornea- aqueous interface. The I and II in the figure depict the wavenumber position used to calculate the $\mathrm{OH} / \mathrm{CH}$ ratio at 3390 and $2945 \mathrm{~cm}^{-1}$ respectively. 
thalmic ointment is applied to dehydrate the cornea and Raman spectroscopic measurements are obtained ten minutes after application. In the rabbit cornea, the tear film will break down after 30 minutes with no blinking of the eye. The measurements are initiated immediately after opening the eyelid, well before the corneal hydration changes would start spontaneously due to lack of blinking. During the measurements of one eye, the other remains closed by using tape.

The water/protein ratio $(\mathrm{OH} / \mathrm{CH})$, defined in the work of Huizinga et al.(21), was calculated to determine the hydration condition of the cornea before and after treatment with Muro 128. This ratio is defined as the Raman intensity of the $\mathrm{OH}$ structure at $3390 \mathrm{~cm}^{-1}$, divided by the Raman intensity of the $\mathrm{CH}$ structure at $2945 \mathrm{~cm}^{-1}$. The $\mathrm{OH} / \mathrm{CH}$ ratio was determined when the probing volume of the confocal probe was approximately 200 micrometers in the cornea stroma from the anterior corneal surface. The focus of this research was the qualitative assessment of the Raman spectra. No absolute water concentration was measured. Before calculating this ratio, the data were smoothed using a Savitsky-Golay 10 point smoothing method. Next, a background spectrum was obtained with no sample in front of the microscope objective. This spectrum was subtracted from the data. The slight remaining baseline slope was removed with a linear two point baseline correction.

\section{Results}

Figure 1A shows an axial scan from the anterior to the posterior border of an untreated cornea, represented in the figure from bottom to top respectively. The scan at the bottom of the figure represents a signal obtained at the air/cornea interface. Each subsequent spectrum was obtained after the probe was axially moved 50 micrometers towards the lens. The last spectrum is obtained at the comea aqueous interface (top spectrum in Figure $1 \mathrm{~A}$ ). Figure IB shows a similar axial scan from the same cornea ten minutes after applying the Muro $128^{\circ}$ to dehydrate the cornea.

Figure 2 shows a typical profile for the changes in the Raman intensity ratio of the $\mathrm{OH} / \mathrm{CH}$ structures as a function of time following topical application of Muro $128^{\otimes}$ in the cornea of a rabbit in vivo. The first measurement (at $\mathrm{T}=0$ ) in figure 2 was obtained immediately before application of the Muro $128^{\circ}$. Ten minutes after application, the measurements were conducted every five minutes.

A summary of the $\mathrm{OH} / \mathrm{CH}$ ratios from six in vivo rabbit corneas obtained without treatment and ten minutes after treatment, is listed in Table I. The Raman spectra used to calculate these ratios are obtained approximately in the middle of the stroma (the fourth spectrum from the bottom in Figure 1). These ratios are calculated with the Student's $T$ Test. The $\mathrm{OH} / \mathrm{CH}$ ratio in normal cornea varied between 1.7 and 1.9 with a mean value of $1.76 \pm 0.09$ while in the dehydrated comea the $\mathrm{OH} / \mathrm{CH}$ ratio varied between 1.1 and 1.4 yielding a mean value of $1.27 \pm 0.13$. Comparison of the averages shows a decrease of about $30 \%$ in the $\mathrm{OH} / \mathrm{CH}$ ratio between the untreated and Muro $128^{\circ}$ treated corneas. 


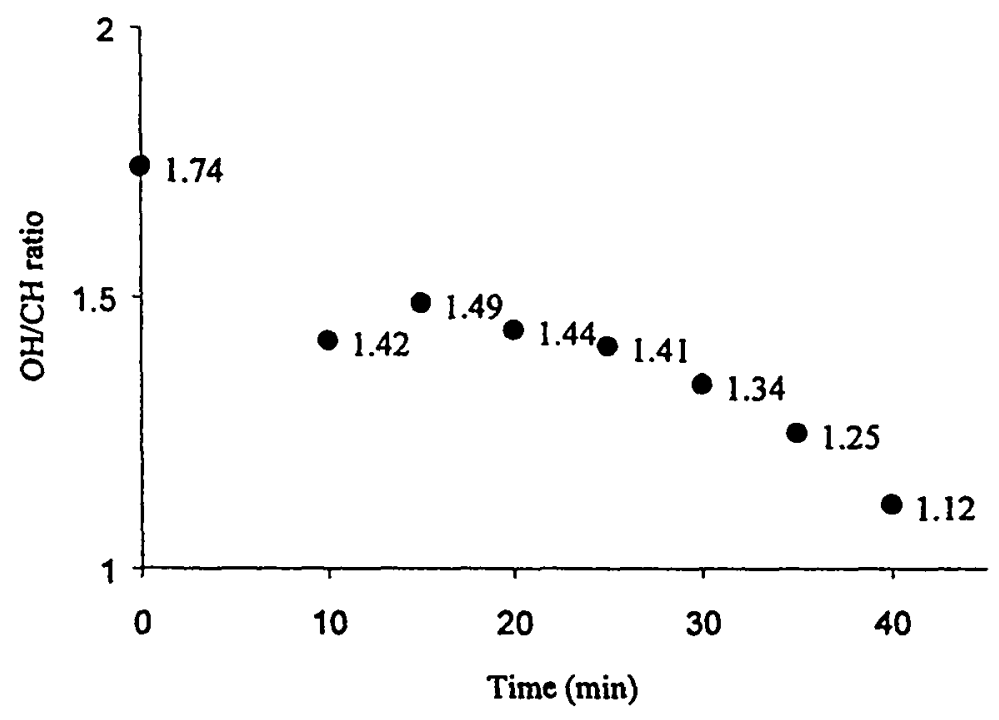

Figure 2. A typical profile for changes observed in the $\mathrm{OH} / \mathrm{CH}$ ratio in the middle of the cornea after Muro 128 is topically applied on the comea of a normal rabbit eye. The measurement at $T=0$ is obtained immediately before applying the Muro. Ten minutes after applying the Muro, the first scan is obtained. The subsequent scans are obtained with intervals of 5 minutes.

\section{Discussion}

Using the Raman spectroscopic technique described above, it is possible to discriminate between different sections of the cornea with various degree of hydration. This is shown in Fig. 1, where different Raman signals arising from the air/cornea boundary, stroma (anterior, medial, posterior), cornea/aqueous humor boundary, and aqueous humor are observed. It should be noted that these spectra are highly reproducible and several axial scans through the tissue gave results similar to those in Figure 1, both before and after Muro $128^{\circ}$ application. This suggests that in these experiments the eye movements were not a major prob-

Table $1 . \mathrm{OH} / \mathrm{CH}$ ratios from normal and treated corneas obtained in vivo from six adult male New Zealand White rabbits.

Normal cornea $(\mathrm{OH} / \mathrm{CH})$

1.70

1.90

1.88

1.69

1.71

1.70

MeantSD
Muro treated cornea (OH/CH)

1.34

1.43

1.22

1.16

1.10

1.42

$1.27 \pm 0.13$ 
lem. Under anesthesia, axial eye movements due to action of the retractor muscle, or lateral eye movements due to the other eye muscles, are reduced. However, eye movements due to breathing vibrations (in order of micrometers) are still possible. Thus, further work needs to be conducted in order to account for the influence of such motions in the reported measurements. The results of the research here show the possibility of monitoring the dehydration changes. Bauer et al.(29) found a decrease of comeal thickness of $50 \mu \mathrm{m}$ in rabbit comeas exposed to an ambient environment after 30 minutes. Siew et al.(37) detected an increase in hydration after excision and storage of human eye bank corneas in medium (corneal swelling).

The current work demonstrates the feasibility for real time monitoring of the hydration changes in the living cornea, as illustrated by the $\mathrm{OH} / \mathrm{CH}$ ratio versus time in Figure 2. The dehydrating effect of Muro $128^{\otimes}$ could also be observed clearly by comparing the $\mathrm{OH} / \mathrm{CH}$ intensities from each spectra obtained within the cornea as shown in Figs. IA and 1B. In particular, the Raman spectra obtained approximately in the middle of the stroma before and ten minutes after treatment clearly show considerable differences as documented by the observed decrease in the $\mathrm{OH}$ structure relative to the $\mathrm{CH}$ peak, indicating the dehydrating effect of Muro 128 ${ }^{\circ}$. Furthermore, a trend was observed in the number of spectra needed to scan through the cornea. A smaller number of 50 micrometer step increments were required to scan through the treated cornea (Fig. 1B) as compared to the untreated cornea (Fig. 1A). This implies that there is a change in corneal thickness after treatment. This is expected since dehydration should result in a decrease in corneal thickness (33).

There are several promising features that this confocal Raman technique provides. First, it is possible to obtain information about physiologic or pathologic ocular tissue without compromising or interfering with the tissue integrity (i.e. no tissue staining or handling is required). It also allows ocular tissue posterior to the cornea to be studied, such as the anterior chamber, aqueous humor, and lens(28). The use of short integration (collection) times allows for in vivo applications to be pursued. The physical properties of the confocal probe, such as the strong divergence of the excitation beam after focussing on the region of interest and the ability to precisely control positioning of the optical probe at different depth in the ocular tissue, makes it virtually impossible to accidentally focus on the retina. The 25 $\mathrm{mJ}$ used in this study is clearly above the retina safety levels for human applications ${ }^{(38)}$. But for the in vivo animal research this model can be used to study the various ocular tissues. Currently we are investigating the use of a more quantum efficient $\mathrm{CCD}$ detector and the use of a longer excitation wavelength $(632.8$ or $647 \mathrm{~nm}$ ) for retinal safety purposes.

Several applications of this method are possible. Examples of these are: Monitoring the healing process following PRK; monitoring corneal grafts; monitoring hydration status of the cornea while exploring new laser wavelengths for refractive keratectomy; determining changes of corneal hydration after the use of topically applied drugs(39); following the reaction of corneal tissue on contact lens usage; and quality control and selection of eye bank eyes ${ }^{(37)}$. Maximum and minimum swelling properties of the corneal stroma could be studied and how stress events like contact lenses or a cataract extraction would interfere with these properties(40-42). It might be possible that the forced dehydration with the Muro $128^{\otimes}$ can be applied as a test for endothelial function. Since it appears related that severity of diabetes effects endothelial cell morphology(43), this method could be used to monitor the diabetes. Application of this technique for pharmacokinetic studies could provide the tool to observe 
in vivo how drugs adhere to the corneal epithelium, penetrate the different corneal layers, or influence the hydration status of the tissue.

\section{Acknowledgments}

We would like to thank Dr. C. Yang for his assistance in handling the rabbits and Dr. N. Bauer for his assistance in obtaining the data. This work was supported in part by The Kempner Foundation, the Department of Energy (grant No. DOE-FG05-91ER61226, A003), and by a Research to Prevent Blindness Development Grant.

\section{References}

1. Rehm WS, and Spangler SG. Am. J. Optometry \& Physiol. Optics 1977: 54: 439.

2. Waring GO III, Bourne WM, Edelhauser HF, and Kenyon KR. Ophthalmol. 1982; 89: 531.

3. Maurice D. J. Physiol. 1957; 136: 263.

4. Fraunfelder FT. Drug induced ocular side effects and drug interactions, 3 rd Edition. Lea \& Febiger: Philadelphia, 1989.

5. Arfa. R. C. Grayson's diseases of the comea, 3 rd Edition. Mosby Yearbook: St. Louis, 1991.

6. Konowal A, Morrison JC, Brown SV, Cooke DL. Maguire LJ, Verdier DV, Fraunfelder FT, Dennis RF, Epstein RJ. Am. J. Ophthalmology 1999; 127: 403.

7. McNamara NA. Brand RJ, Polse KA, and Boume WM. Invest. Ophthalmol. Vis. Sci. 1998; 39: 3.

8. Salz JJ, Maguen E, Nesburn AB, Warren C, Macy JI, Hofbauer JD, Papaioannou T, and Bertin M. Ophthalmology 1993: 100: 873 .

9. Dougherty PJ, Wellish KL, and Maloney RK. Am. J. Ophthalmology 1994; $118: 169$.

10. Oshika T, Klyce SD, Smolek MK. and McDonald MB. J. Cataract Refract. Surg. 1998; $24: 1575$.

11. Ichijima H, Petroll WM, Jester JV, Barry PA, Andrews PM. Dai M, and Cavanagh HD. Comea 1993; 12: 369.

12. Petroll WM, Cavanagh HD, and Jester JV. J. Microscopy 1993; 170: 213.

13. Wilson G, O'Leary DJ. Vaughan W. Invest. Ophthalmol. Vis. Sci. 1984; 25: 1105.

14. Turss R, Friend J, Reim M. Dohlman CH. Ophthalmic Res. 1971: 2: 253.

15. Seiler T, Trahms L, and Wollensak J. Graefes Arch. Clin. Exp. Ophthalmol. 1982; 219: 287.

16. Kiener C, Cavellier JF, Binaghi M. Renard G, Adenis JC. J. Fr. Ophtalmol. 1984: 7: 609.

17. Pallikaris IG, Ginis HS, Kounis GA, Anglos D, Papazoglou TG, and Naoumidis LP. J. Refract.Surg. 1998; $14: 655$.

18. Castoro JA. Bettelheim AA, Bettelheim FA. Invest. Ophthalmol. Vis. Sci. 1988; $29: 963$.

19. Mathies R, and Yu N-T. J. Raman spectroscopy 1978: 7: 349.

20. Yu N-T, Kuck JF, and Askren CC. Curr. Eye Res. 1982; 1: 615.

21. Huizinga A. Bot ACC. de Mul FFM, Vrensen GFJ, and Greve J. Exp. Eye Res. 1989; $48: 487$.

22. Carey PR. Quarterly Reviews of Biophysics II 1978; 3: 309.

23. Sulkes M, Lewis A, and Marcus M. Biochemistry 1978; 17:4712.

24. Sebag J. Nie S, Reiser K. Charles MA, and Yu N-T. Invest. Ophthalmol. Vis. Sci. 1994; 35: 2976.

25. Bemstein PS, Yoshida MD, Katz NB, McClane RW, and Gellermann W. Invest. Ophthalmol. Vis. Sci. 1998; $39: 2003$.

26. Wicksted JP. Erckens RJ, Motamedi M, and March WF. Applied Spectroscopy 1995: 49: 987.

27. Erckens RJ, Motamedi M, March WF, and Wicksted JP. J. Raman Spectroscopy 1997; 28: 293.

28. Jongsma FHM, Erckens RJ, Wicksted JP. Bauer NJC. Hendrikse F, March WF, and Motamedi M. Optical Engineering 1997: 36: 3193. 
29. Bauer NJC, Wicksted JP, Jongsma FHM, March WF, Hendrikse F, and Motamedi. M. Invest. Ophthalmol. Vis. Sci. 1998; 39: 831.

30. Bauer NJC, Hendrikse F, March WF. Cornea 1999; 18:483.

31. Erckens RJ, March WF, Jongsma FJ, Wicksted JP. Hendrikse F, Smit EE, Motamedi M. In Press in Journal of Cataract and Refractive Surgery.

32. Berman ER. Biochemistry of the eye. Plenum. New York, 1991.

33. Fatt 1, and Weissman B. Physiology of the eye, 2nd ed. Butterworth-Heinemann, 1992.

34. Hedbys B, and Mishima S. Exp. Eye Res. 1966; 5: 221.

35. Prince J. The rabbit in eye research. C.C. Thomas publishers: Springfield, Illinois, 1964.

36. Mees C, James T. The theory of the photographic process. The Macmillian Company: New York, 1966.

37. Siew DCW. Clover GM, Cooney RP, and Wiggins PM. J. Raman Spectroscopy 1995: $26: 3$.

38. ANSI. American National Standards Institute. Safe use of lasers. In: ANSI standard Z 136.1. Laser Institute of America. Orlando, 1993.

39. Mitra AK. Ophthalmic drug delivery systems. In Series: Drugs and the pharmaceutical sciences; Vol. 58. Marcel Dekker Inc.: New York, 1993.

40. McMahon TT, Polse KA, McNamara N, and Viana MA. Optom Vis Sci. 1996; 73: 184.

41. Saini JS, and Mittal S. Ophthalmic Surg Lasers 1996; 27: 1000.

42. Odenthal MT, Nieuwendaal CP. Venema HW, Oosting J, Kok JH, and Kijlstra A. Invest. Ophthalmol. Vis. Sci. 1999; 40: 312.

43. Weston BC, Bourne WM, Polse KA. and Hodge DO. Invest. Ophthalmol. Vis. Sci. 1995; 36: 586. 


\section{Chapter 7}

\section{Noninvasive in vivo assesment of rabbit lens hydration using confocal Raman spectroscopy}

Roel J. Erckens, 1.2 Franciscus H. M. Jongsma,' James P. Wicksted, ${ }^{3}$ Fred Hendrikse, 1 Wayne F. March,2 Massoud Motamedi²

1 Department of Ophthalmology, Academic Hospital Maastricht, Maastricht. The Netherlands.

2 Laser \& Spectroscopy Program of the Center for Biomedical Engineering and the Department of Ophthalmology and Visual Sciences. University of Texas Medical Branch Galveston. Galveston, Texas 77555-0456, U.S.A.

3 Department of Physics, Center for Sensors \& Sensor Technologies, Oklahoma State University. Stillwater, Oklahoma 74078-3072, U.S.A.

Key Words: Cataract models. lens proteins, hydration, Raman spectroscopy, in vivo rabbit eye.

Supported by an unrestricted grant from RPB. 


\section{Abstract}

Purpose: To develop a noninvasive technique to assess the local hydration of the eye lens for longitudinal survival studies designed to follow the biochemical changes that may occur in the lens.

Methods: A confocal Raman spectroscopic probe was developed and interfaced to a visible laser source and CCD camera. The system was used to detect Raman signals from the eyes of six anesthetized rabbits. The discrete Raman spectra of the lens with peak assignments to $\mathrm{OH}$ modes at $3390 \mathrm{~cm}^{-1}$ (water) and CH modes at $2935 \mathrm{~cm}^{-1}$ (proteins) were used to calculate the $\mathrm{OH} / \mathrm{CH}$ ratio and assess the change in the water content of the lens.

Results: The confocal probe allows for spatial mapping of the lens hydration with a high spatial resolution using low energy probing light. Raman spectra were obtained using an integration time of either $1 \mathrm{~s}$ or $0.1 \mathrm{~s}$. The argon laser power of was either $7 \mathrm{~mW}$ or $1 \mathrm{~mW}$. The integration depth was $125 \mu \mathrm{m}$ or $1000 \mu \mathrm{m}$. The $\mathrm{OH} / \mathrm{CH}$ ratio showed typical values from 0.49 at $1 \mathrm{~mm}$ depth in the outer cortex to 0.38 in the lens nucleus.

Conclusions: The Raman spectroscopic technique could be used for noninvasive optical sectioning of the lens that allows to assess the $\mathrm{OH} / \mathrm{CH}$ ratio in the lens tissue. The $\mathrm{OH} / \mathrm{CH}$ ratio could be determined with an integration time as short as $0.1 \mathrm{~s}$ and $1 \mathrm{~mW}(0.1 \mathrm{~mJ})$. 


\section{Introduction}

In ophthalmology, determining the cause of cataract formation poses many challenges to obtain information on this disease since cataract is the most important cause of preventable blindness in the world. It is estimated that in the U.S.A. 8 million people each year are affected by cataract related eye problems and 1.3 million people need cataract procedures(1). These numbers show the need to understand the etiology of cataract formation. In recent years significant efforts have been devoted to the development of new noninvasive and nondestructive diagnostic techniques for early detection of changes in biochemical composition or obtain information on the morphology of ocular tissues $(2)$. To obtain information on molecular identification or changes in chemical structure is feasible with spectroscopic techniques. Among them there is absorption, fluorescence and scattering spectroscopy. To be used noninvasively, fluorescence and scattering spectroscopies are more suitable to use in tissues.

Raman Spectroscopy is a scattering spectroscopic technique that offers the potential for noninvasive assessment of ocular tissue(3). The unique optical properties of the eye provide an excellent opportunity to use visible or near IR light Raman spectroscoptic(4) techniques to probe various tissues of the eye. In $1982 \mathrm{Yu}^{(5)}$ showed the first Raman spectra obtained from the lens in the living rabbit. However, the use of Raman spectroscopy to study the change in hydration and/or protein structure of the lens has mainly been described(4) for the in vitro situation. In this study, Raman spectroscopy was used to obtain the hydroxyl $(\mathrm{OH})$ stretching mode from water in the lens and the carbon-hydrogen $(\mathrm{CH})$ stretching mode from lens protein. The $\mathrm{OH}$ intensity at $3390 \mathrm{~cm}^{-1}$ and the $\mathrm{CH}$ intensity at $2935 \mathrm{~cm}^{-1}$ were used to calculate the $\mathrm{OH} / \mathrm{CH}$ ratio and hence the changes in hydration in the living rabbit.

To determine the hydration status in different sections of the eye lens in vivo while using the Raman spectroscopic technique, several aspects need to be addressed: (i) if the optical path length of the probing beam is too large, it is difficult to interpret the obtained spectra since Raman signals arising from adjacent tissues would be added together; (ii) the fluorescence of the target tissue can mask the Raman signals; (iii) The power density of the probing beam has to be within certain limits, not only to prevent denaturation of the proteins in the target tissue, but also to prevent light damage to the retina from the diverging laser beam.

In this study, we examined changes in hydration from rabbit eye lenses in vivo. This was accomplished by measuring the Raman signal arising form the water (OH stretching mode) and protein $(\mathrm{CH}$ stretching mode) structures as the lenses were probed from the anterior (cortex) towards the more posterior (nuclear) regions of the lens. By using young healthy rabbits, no significant fluorescense was present from the lenses when the $514.5 \mathrm{~nm}$ line of the argon excitation laser was used. To differentiate between Raman signals arising from different regions in the lens while maintaining a good spatial resolution, we utilize the confocal detection system developed by Jongsma et al(6). The use of low laser powers, short exposure times and confocal integration depths were studied in order to determine the best combination for eye safe experiments. 


\section{Materials}

Figure 1 shows the confocal probe design: The $514.5 \mathrm{~nm}$ laser light from a CR-4 Argon laser (Coherent Laser Group, Palo Alto, CA) was used as excitation source. The collimated laser beam was directed through a holographic bandpass filter (Omega Optics, Brattleboro, Vermont) designed to work at an angle of approx. 45 degrees. This collimated and filtered beam was then focused on the sample using a Planachromat (ausJena, Jena, Germany) long distance microscope objective with a magnification and numerical aperture of 25 and 0.5 respectively. The Raman and Rayleigh backscattered light arising from the sample was collected through the same long distance microscope objective and bandpass filter. This collected light was send through a holographic notch filter at $514.5 \mathrm{~nm}$ (Kaiser Optical Systems, Ann Arbor, MI) to block Rayleigh scattered light from the sample, and passed through a F $0.95 / 25 \mathrm{~mm}$ large aperture lens (Navitar, Rochester N.Y.) to focus the Raman light on a 50 or $400 \mu \mathrm{m}$ diameter silica fiber, each $2 \mathrm{~m}$ in length. The fiber acted as the pinhole in the confocal configuration. In tissue the integration depth of the current design is about 125 or $1000 \mu \mathrm{m}$ with the 50 or $400 \mu \mathrm{m}$ diameter fiber respectively.

The fiber was coupled into a single grating spectrometer model 500M (Spex Industries, Edison, NJ), equipped with a liquid nitrogen cooled Charge Coupled Detector array (CCD). Raman spectroscopic data of ten eye lenses from five adult male New Zealand White rabbits were obtained. The animals were anesthetized using ketamine and xylazine and placed in a stereotactical holder with height control and treated according to the ARVO resolution on the use of animals in research. The eyes were kept open using a speculum and moistered with balanced salt solution to prevent cornea dehydration. The animals were sacrificed after the measurements since the total data collection obtained using different expererimental settings could have resulted in retinal damage and the required prolonged anesthesia could have resulted in systemic damage.

The Raman spectral features were obtained in the higher spectral region from 2600 to 3600 $\mathrm{cm}^{-1}$ along the optical axis of the eye lens. The water/protein ratio $(\mathrm{OH} / \mathrm{CH})$ was calculated

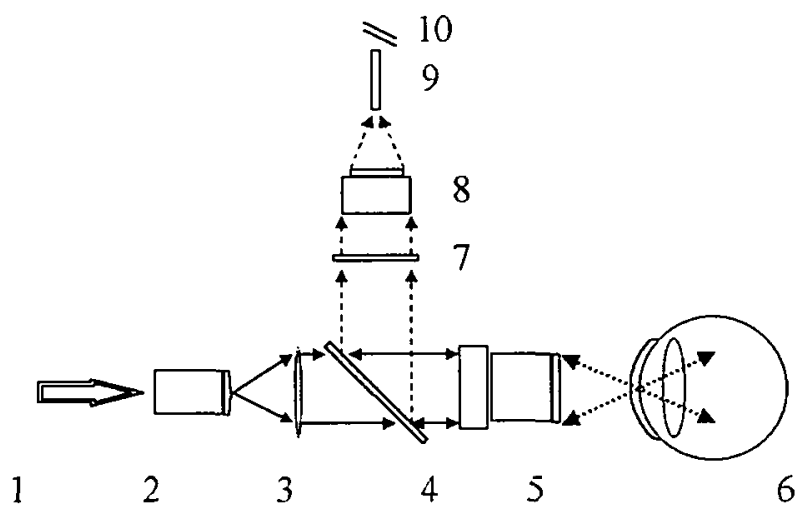

Fig. 1. l=laser beam; $2 \& 3$ =collimator; $4=$ bandpass filter; $5=$ long distance microscope objective; $6=$ eye; $7=$ notch filter; $8=$ high speed lens; $9=$ fiber; $10=$ towards spectrometer. 
to determine the hydration condition in different regions of the lens. This ratio is defined as the Raman intensity of the $\mathrm{OH}$ structure at $3390 \mathrm{~cm}^{-1}$, divided by the Raman intensity of the $\mathrm{CH}$ structure at $2935 \mathrm{~cm}^{-1}$. Before calculating this ratio, the data were smoothed using a Savitsky-Golay 10 point smoothing method. The base line of each spectrum was estimated corresponding to the work of Iriyama et al.(7)

\section{Results}

Figure 2a shows typical Raman spectra obtained in the cortex region at a depth of $500 \mu \mathrm{m}$ from the front lens area and in the center of the nuclear region. Visible are the Raman features from $\mathrm{CH}$ stretching modes at $2935 \mathrm{~cm}^{-1}, \mathrm{CH}$ stretching modes at $3064 \mathrm{~cm}^{-1}$ and the broad $\mathrm{OH}$ stretching mode (between about 3200 and $3500 \mathrm{~cm}^{-1}$ ) with the maximum around $3390 \mathrm{~cm}^{-1}$. Laser power $7 \mathrm{~mW}$, integration time $1 \mathrm{~s}$, integration depth $125 \mu \mathrm{m}$, probe increments $100 \mu \mathrm{m}$. Figure $2 \mathrm{~b}$ shows the averaged $\mathrm{OH} / \mathrm{CH}$ ratio calculated from the in vivo Raman spectra of the ten normal rabbit eye lenses collected in the cortex and nuclear regions. A gradual decrease in the $\mathrm{OH} / \mathrm{CH}$ ratio is observed while scanning towards the lens nucleus. The increments of the probe are $100 \mu \mathrm{m}$ in the first millimeter in the anterior as well the posterior cortex region of the lens. Between these regions, the increments of the probe are one millimeter. At $0.5 \mathrm{~mm}$ depth in the cortex the $\mathrm{OH} / \mathrm{CH}$ ratio is 0.75 (SD 0.047); at $1 \mathrm{~mm}$ depth in the cortex the $\mathrm{OH} / \mathrm{CH}$ ratio is 0.49 (SD 0.029); and at $3.5 \mathrm{~mm}$ in the nucleus the $\mathrm{OH} / \mathrm{CH}$ ratio is 0.38 (SD 0.047). Laser power of $7 \mathrm{~mW}$, and an integration time of $1 \mathrm{~s}$.

Figure 3 shows the actual Raman spectra while scanning from the anterior to the posterior regions of the lens. The probe integration depth was $1 \mathrm{~mm}$, probe increments were also $1 \mathrm{~mm}$. The laser power on the sample was $1 \mathrm{~mW}$ with an integration times of $0.1 \mathrm{~s}$.

The relative water content divided by the protein content (I $3390 \mathrm{~cm}^{-1} / \mathrm{I} 2935 \mathrm{~cm}^{-1}$ ) is shown on the right side of the figure. These values were similar as those in Fig. 2 and are

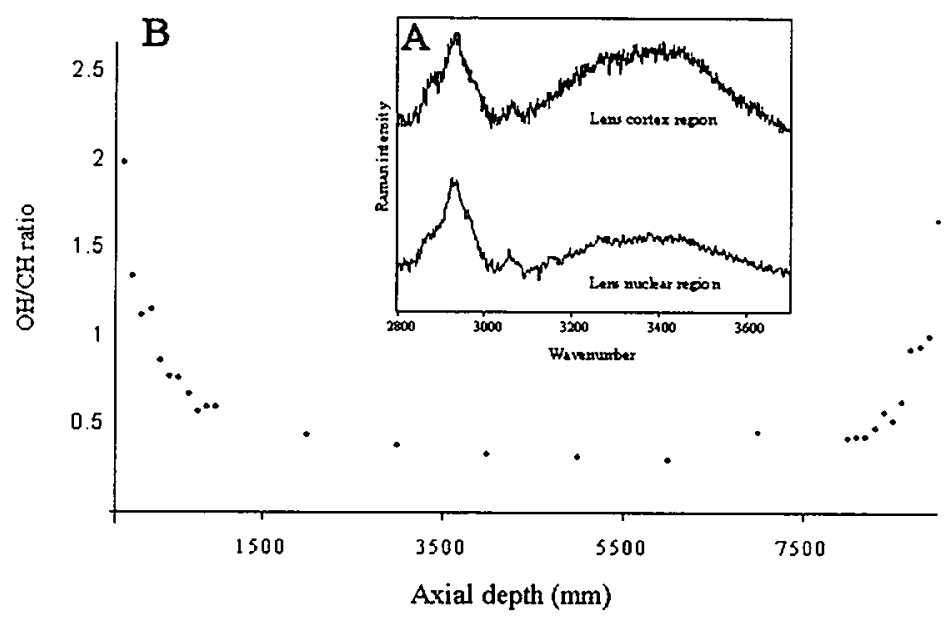

Fig. 2. Raman spectra (A) from lens cortex and nucleus; $O H / C H$ ratio (B) through lens. 


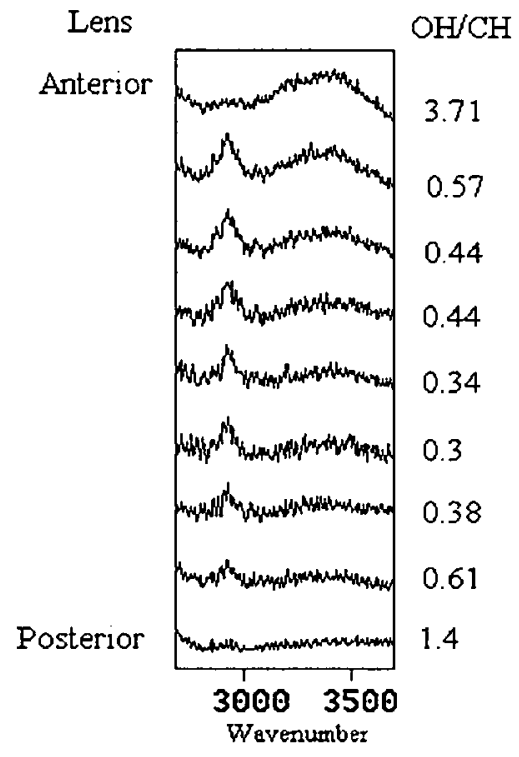

Fig. 3. Raman spectra while scanning through lens. Laser power $1 \mathrm{~mW}$, integration time $0.1 \mathrm{~s}$.

depicted on the right side of the spectra. While scanning from the cortex towards the nuclear region the decrease in the $\mathrm{OH} / \mathrm{CH}$ ratio can be observed. However, due to the refractive power of the long distance microscope objective with the additional optical properties of the eye, this probe configuration is less suitable to map the lens all the way into the vitreous humor.

\section{Discussion}

Using the confocal probe configuration it was possible to detect and distinguish the Raman spectra of the lens in an in vivo animal model with low laser power. As can be seen from figure 2 , the water gradient decreases while the protein gradient increases from the cortex moving towards the nuclear lens region. The water versus protein ratio shows the same tendencies as were described earlierin other studies from eye lenses ${ }^{(4)}$. Furthermore the $\mathrm{OH} / \mathrm{CH}$ ratio found in this study is comparable with the values Ozaki(8) found for rabbits of the age group we used (about 2 years old). The numerical apertue of the pupil limits the use of other excitation/collection angles other than 180 degrees if tissues located behind the iris, like the lens, are to be investigated. Therefore, the probe in 180 degrees configuration seems to be the best useable setup since the excitation and scattered light both travel through the same lens and under all conditions maintain their exact alignment.

The area of the diverging laser beam on the retina is about $1 \mathrm{~cm}^{2}$, while the laser beam is focussed in the nuclear lens region. According to the ANSI laser retinal safety standards(9), this would be in the safe region. In this ANSI standard, a maximum of $1.8 \mathrm{~mJ} / \mathrm{cm}^{2}$ of laser 
energy may be applied. The use of $0.1 \mathrm{~mJ}$ would produce no light induced damage to the retinal tissue. However the use of $1 \mathrm{~mJ}$ improves the signal quality as can be seen in Fig.3a and is still in the safety region to prevent retinal damage. So this technique could be used to perform longitudinal studies in the animal model without damaging the retina or the need to sacrify the animal after the experiment. Since there is no contact of the probe with the eye tissue, anesthesia can be adjusted according to the noninvasive nature and short duration of the measurements. This might improve the health and comfort of the animal. The possibility to follow the change in water or protein concentration in the lens of the living animal might be very promising. It could give more insight in the physiological processes in lens metabolism, and the pathological conditions like cataract formation in the animal model. Future research goals are: 1. Follow in the living eye the age related cataract formation. 2 . Follow up on anti-cataract drugs in experimental animals(10-12). In addition, for understanding the formation of toxic cataracts like the steroid-induced or by the use of the cardiac drug amiodarone, it might be possible to use this technique for real time monitoring in the experimental animal model. 3. Follow induced cataract formation after radiation with ultraviolet light(13).

With this probe it would be possible to obtain Raman spectra of eyes from smaller animals like rats.

The use of the 1 or $0.1 \mathrm{~mJ}$ brings this technique also closer to the use in investigation the cataract formation in the living human. It is believed that besides 'aging' of the lens, also risk factors(14) like ultraviolet-B radiation, drugs, diabetes, dehydration, alcohol and nicotine play an important role. Furthermore, at specific circumstances a cataract may develop: Formation of cataract after ocular penetration of a foreign body(15) entrapped in the lens, or after blunt trauma like from a paintball gun(16); the use of silicone oil or intravitreal gasses used in retina surgery(17) (pars plana vitrectomy); and after silicone IOL implantation in phakic eyes to treat high myopia(18).

\section{References}

1. Basic and Clinical Science Course Section I1. Lens and Cataract. American Academy of Ophthalmology 1996-1997.

2. Masters B. Noninvasive diagnostic techniques in Ophthalmology. New York: Springer-Verlag, 1990.

3. Bauer N.J.C. Potential applications of Confocal Raman spectroscopy in Ophthalmology. Thesis University of Maastricht. the Netherlands, 1999.

4. Erckens RJ, Jongsma FH, Wicksted JP, Hendrikse F, March WF, Motamedi M. Raman spectroscopy in ophthalmology: From experimental tool to applications in vivo. Review. In Press in Lasers in Medical Science.

5. Yu NT, Kuck J. Askren C. Laser Raman Spectroscopy of the Lens in situ, measured in an anesthetized rabbit. Current Eye Research. 1982;1;10:615-618.

6. Jongsma. F. H. M., Erckens. R. J., Wicksted. J. P., Bauer, N. J. C.. Hendrikse. F., March. W. F. and Motamedi. M. (1997). A confocal Raman spectroscopy system for non-contact scanning of ocular tissues: An in vitro study. Optical Engineering 36, 3193-3199.

7. Iriyama K, Mizuno A, Ozakj Y, Itoh K and Matsuzaki H. An application of laser Raman spectroscopy to the study of a hereditary cataractous lens; on the Raman band for a diagnostic marker of cataractous signatures. Current Eye Research $1982 / 1983 ; 2: 489-492$. 
Mizuno A. Molecular aging of lens crystallins and the life expectancy of the animal. Ageanges studied in situ by Raman spectroscopy. Biochimica et Biophysica Acta. 1992;1121: : i). American National Standards Institute. Safe use of lasers. In: ANSI standard Z 136.1. 1 lando.

Iran P, Henein M, Ali A. Varma S. Attenuation of sugar cataract by ethyl pyruvate. Mol Ce 2): $103-109$.

:ojima M, Sasaki $\mathrm{K}$. Effect of vitamin $\mathrm{E}$ eye drops on naphthalene-induced cataract in rats. 5(4):345-50.

ardenas H, Caperell-Grant A. Grubbs C. Protective effects of estrogen in a rat model of agi ad Sci USA 1999;3;96(16):9328-32.

irensen G. van Marle J, Lofgren S, Soderberg P. Repair in the rat lens after threshold ultra t Ophthalmol Vis Sci 2000:41(1):204-12.

idemiology of age-ralated cataract. Eye 1999;(Pt 3B):445-8.

oyd S. Feldman F. Heathcote J, Steiner M, Billson F. Intralenticular foreign bodies: case $\mathrm{rC}$ thalmic Surg 1995;26(3):250-2.

at S. Eye injuries associated with paintball guns. Int Ophthalmol 1998-99;22(3):169-73.

ver $P$. Silicone oil in the treatment of massive preretinal retraction. I. Results in 105 eyes. I i55-60.

?. Wehler T. Busin M. High incidence of cataract formation after implantation of a silicone ic. highly myopic eyes. Ophthalmology 1999; 106(9): 1651-5. 


\section{Chapter 8}

\section{Raman spectroscopy: noninvasive determination of silicone oil in the eye. Potential applications for intraocular determination of biomaterials.}

Submitted.

Roel J. Erckens, MD', Kamran Hosseini, MD2, Wayne F. March MD², Franciscus H. M. Jongsma, PhD' ${ }^{1}$, James P. Wicksted, PhD', Helen K. Li, MD'2, Fred Hendrikse, MD $\mathrm{PhD}{ }^{1}$

1 Department of Ophthalmology. Academic Hospital Maastricht. Maastricht. The Netherlands.

2 Laser \& Spectroscopy Program of the Center for Biomedical Engineering and the Department of Ophthalmology and Visual Sciences, University of Texas Medical Branch Galveston, Galveston, Texas 77555-0456, U.S.A.

3 Department of Physics, Center for Sensors and Sensor Technologies, Oklahoma State University, Stillwater, OkJahoma 74078-3072, U.S.A.

Reprint requests: Roel J Erckens, M.D.

Department of Ophthalmology. Academic Hospital Maastricht,

P. Debeyelaan 25, Postbus 5800,6202 AZ Maastricht, The Netherlands.

Phone: 31-0621203131, Fax: 31-433875343,E-mail: re@wxs.nl 


\begin{abstract}
Purpose. To show in a case study that Raman spectroscopic analysis for noninvasive determination of intraocular molecular structures of tissue or artificial materials is feasible.

Material and methods. Raman spectroscopic recordings were obtained using a confocal detection probe with an adjustable integration depth to resolve a small droplet $(0.75 \mathrm{~mm})$ of silicone oil noninvasively in an animal model (rabbit eye) at different power settings. After these measurements, the power was reduced to $95 \mu \mathrm{W}$ for a safe measurement in the human eye.

Results. Raman spectral features indicative of silicone oil were detected at $2906 \mathrm{~cm}-1$ and $2965 \mathrm{~cm}^{-1}$. In the rabbit model, silicone oil could be distinguished from cornea or lens tissue due to the typical Raman spectrum. Even with a safe $95 \mu \mathrm{W}$ power setting, silicone oil could be resolved in the anterior chamber of a patient's eye with a centrally opaque cornea. Conclusion. Due to the strong intensity and specificity of the Raman signal of silicone oil, even small amounts, for instance, those present in the anterior chamber of the eye, can be detected within a time frame of seconds using a safe laser power setting. With these results, an initial step is made in exploring the use and eventually the clinical application of the confocal Raman spectroscopic technique to obtain in vivo information on biological or artificial molecules in the living eye.
\end{abstract}




\section{Introduction}

Intraocular biomaterials are extensively used(1), and well known examples include the artificial intraocular lenses (IOLs), and the vitreous substitutes like silicone oil. The latter is used during a pars plana vitrectomy (PPV), a surgical procedure(2) that is used in the treatment of a retinal detachment, a proliferative vitreous reaction (PVR), a proliferative diabetic retinopathy (PDR), a macular hole or a trauma. Silicone oil(3-4) is used to tamponade the retinal tissue(2). In past years, an increase in the use of silicone oil by the vitreoretinal surgeon while performing a PPV has been noticed. This is due to new treatment options like a macular rotation(5-7) in the case of aged-related macular degeneration, or due to new insights like the use of silicone oil instead of intraocular gases in treatment of a macular hole(8-9), or treatment of an AIDS-related retina detachment(10). However, after the PPV, postoperative complications related to the use of the silicone oil may develop. These include(11-15) cataract formation in the phakic eye, pupillary block glaucoma, closure of inferior iridectomy, or keratopathy, especially in the aphakic eye, where the silicone oil may touch the cornea inducing corneal decompensation and subsequently endothelial cell loss(16-17). Furthermore, blockage of the anterior chamber angle with multiple silicone oil droplets can inhibit the outflow of the aqueous humor resulting in glaucoma(18-19).

A complete filling of the anterior chamber with the silicone oil is frequently difficult to determine at slit lamp examination particularly if the cornea is opaque, thus preventing observation of convection movements of particles in the anterior chamber or other visual signs indicative of the presence of silicone oil.

The aim of this study was to investigate a noninvasive and therefore safe technique to obtain information on the silicone oil in the anterior chamber of the eye. In general, noninvasive and nondestructive tissue or material determination requires the use of spectroscopic techniques. Of the optical spectroscopic techniques, it had been proven that Raman spectroscopy ${ }^{(20)}$ is a valuable tool to determine the molecular composition of biological materials(21-24). The Raman signal is formed by shifts of wavelengths of the incident light, which are related to the frequencies of the modes of vibrations of the light scattering molecules. A confocal Raman excitation and collection probe has been developed by our group(25). Initially, the cornea hydration was determined with this system in the blind human eye using unsafe excitation light on the retina(26). In another study using this confocal probe, the molecular structure of an implanted intraocular lens (IOL) could be determined in a patient using safe laser power excitation levels(27). This confocal probe was also used in this study to detect the silicone oil in the anterior chamber of the eye of a living rabbit, and in a patient after a PPV.

\section{Materials and methods}

The Raman experimental setup with the confocal probe(25) is shown in Figure 1A. The main features of the probe are a noncontact measurement at a working distance of $12 \mathrm{~mm}$ by a long working distance microscope objective (LDMO) with a high light gathering power, and the ability to control the integration depth. This was achieved by a confocally placed collection fiber with a diameter of $400 \mu \mathrm{m}$ yielding an integration depth of about $1 \mathrm{~mm}$ (Fig. 


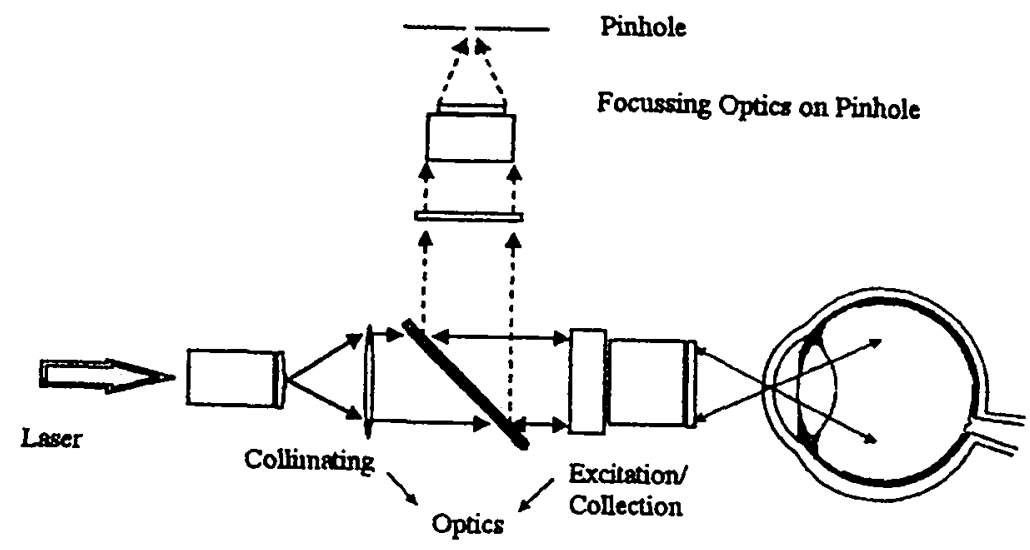

Fig. 1A. Raman experimental setup for in vivo measurements of silicone oil in the anterior chamber of the eye.

1A). A holographic bandpass filter (BP) allows the excitation light to pass, but reflects the Raman scattered light toward the fiber. An argon laser emitting at $514.5 \mathrm{~nm}$ was used to excite the Raman spectra.

To determine the Raman signal of the silicone oil, a cuvette was filled with silicone oil with a viscosity of 5000 centistokes (AdatoSil $5000^{\mathrm{TM}}$, Bausch\&Lomb Surgical, Heidelberg, D), and Raman spectra were recorded in $1 \mathrm{~s}$ using only $12 \mathrm{~mW}$.

For the animal study two New Zealand white rabbits were anesthetized with ketamine and xylazine and treated according to the ARVO resolution on the use of animals in vision research. The silicone oil was introduced in the anterior chamber by means of an injection at the limbus. In one eye of each animal, the amount of silicone oil injected was sufficient to obtain an almost complete filling of the anterior chamber. For these in vivo measurements, the laser power was reduced to $5 \mathrm{~mW}$ and $1 \mathrm{~s}$ integration time. For the experiment in the fellow eye, the laser power was further reduced to $0.5 \mathrm{~mW}$ and $1 \mathrm{~s}$ integration time. In this eye, a silicone oil bubble with a diameter of about $0.75 \mathrm{~mm}$ was injected at the limbus in the superior anterior chamber. The Raman spectrum was obtained by visual focusing on the cornea in front of the bubble. By moving the focusing beam through the different tissue layers, the cornea, silicone oil and lens could be identified respectively using their specific Raman spectra. In this eye, the pupil was dilated using a mydriatic agent (Mydfrin) to obtain the Raman spectrum of the lens. The animals were sacrificed after the measurements with a lethal injection of intracardiac sodium pentobarbital since both eyes had been investigated.

For the human study: At the time there was one 25 year old male patient with silicone oil in the anterior chamber and a central opaque cornea. The patient had a traumatic event 7 months earlier due to an exploding glass bottle. He received a PPV with silicone oil. The lens had to be removed during the PPV. He developed an early keratopathy, possibly due to corneal irritation from the silicone oil. The current vision in this eye was "count fingers at 1 foot". The intraocular pressure was $11 \mathrm{~mm} \mathrm{Hg}$. The study was performed under the 
approval of the Institutional Review Board. The patient gave Informed Consent after the study protocol was explained. For proper positioning of the patient, a modified chin rest and forehead holder from a slit lamp were used and mounted on an optical bench to support the head of the patient, which allowed for height control. Since the cornea of the patient was opaque in the center, the Raman spectrum was obtained on a fairly clear part of the inferior cornea as shown in Figure 1B. The laser power setting was $95 \mu \mathrm{W}$ and an integration time of 1s. The American National Standard for the Safe Use of Lasers(28) gives maximum permissible exposure (MPE) to prevent laser light damage in tissues, including the retina. Utilizing these safety standards, it is estimated that for the human, the MPE at a wavelength of $514.5 \mathrm{~nm}$ should be below $3 \mathrm{~mJ}$ if the illuminated retina area is about $2.7 \mathrm{~cm}^{2}$ with an exposure time equal or longer than 1 second(29). This illuminated area occurs in this probe design and is valid while focusing at the cornea. By focusing in the aqueous humor adjacent to the cornea with $95 \mu \mathrm{W}$ and $\mathrm{ls}(95 \mu \mathrm{J})$, the exposure is well below this value.

\section{Opacity}

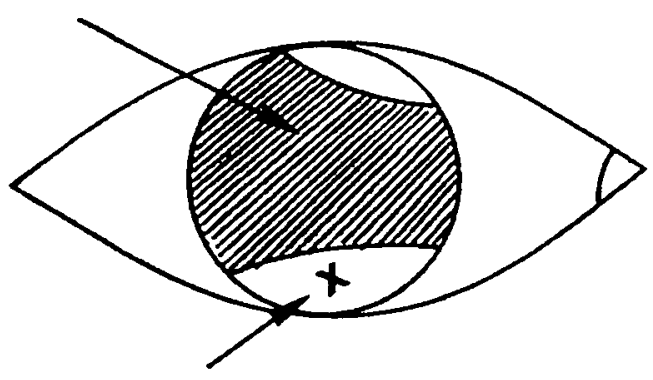

\section{Probing area}

Fig. IB. Human patient eye showing the opaque comea and probing area for detecting the silicone oil.

\section{Results}

The chemical structure and the excellent Raman spectra of 'pure' silicone oil in a cuvette are shown in Figure 2. Typical Raman peak positions are located at $488 \mathrm{~cm}^{-1}, 708 \mathrm{~cm}^{-1}, 1409$ $\mathrm{cm}^{-1}, 2906 \mathrm{~cm}^{-1}$ and $2965 \mathrm{~cm}^{-1}$. Since the Raman intensity of the 2906 and $2965 \mathrm{~cm}^{-1}$ peaks are much stronger than the other peaks, these latter 2 positions were used to identify the silicone oil in vivo. Laser power was $12 \mathrm{~mW}$ and $1 \mathrm{~s}$ integration time.

Figure 3 shows the Raman spectrum of a complete silicone oil filling of the anterior chamber in the rabbit. Laser power was $5 \mathrm{~mW}$ and $1 \mathrm{~s}$ integration time. Even at this reduced power, the good signal to noise ratio provides latitude for the use of lesser energy. Therefore, we further reduced the power to $0.5 \mathrm{~mW}$ for the next experiment (Fig. 4).

Figure 4 shows Raman spectra of the cornea, a small silicone oil bubble in the anterior chamber, and the lens. The oil bubble with a diameter of about $0.75 \mathrm{~mm}$ was located in the 


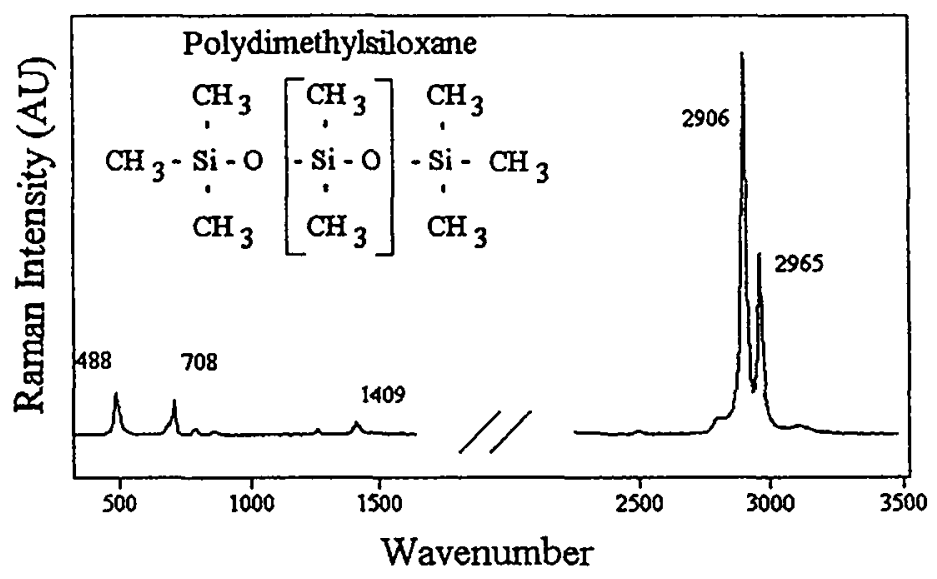

Fig. 2. Structure of silicone oil and Raman spectral features.

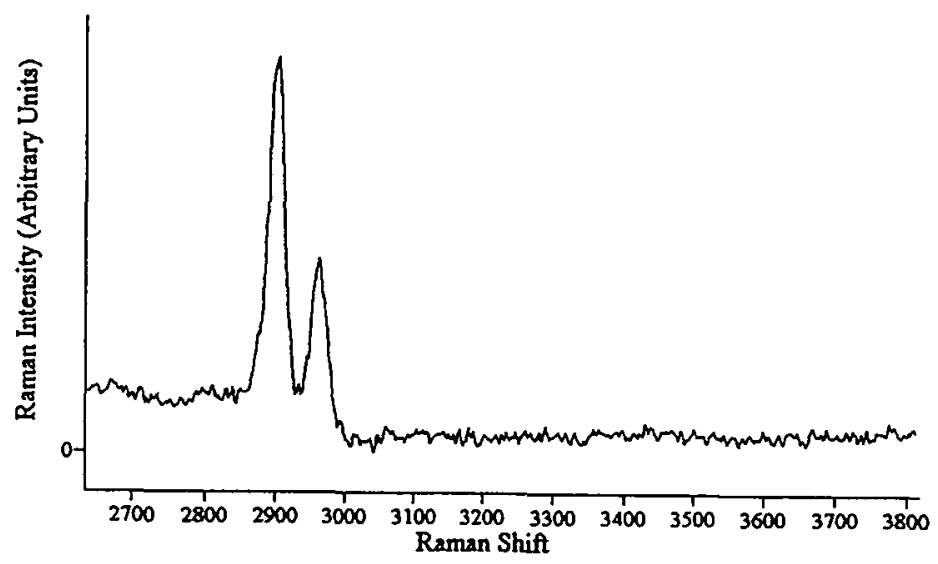

Fig. 3. In vivo Raman spectrum of the anterior chamber of the rabbit eye injected with silicone oil.

superior anterior chamber. Due to the power reduction and the bubble size, which reduces the integration length, the quality of the signal is somewhat less than in Figure 3. The Raman spectrum was obtained by visual focusing on the bubble with the laser beam. The * is the more pronounced position at $3060 \mathrm{~cm}^{-1}$ (Aromatic $\mathrm{CH}$ vibration) indicative of lens tissue. As can be seen of the cornea and lens Raman spectra, there is contribution of water content in these tissues seen as the more broad spectral feature with an intensity maximum around $3390 \mathrm{~cm}^{-1}$. The silicone oil has no significant contribution of a water signal (even more readily seen in Figure 2). Laser power $0.5 \mathrm{~mW}$ and $1 \mathrm{~s}$ integration time. 
Figure 5 shows a Raman spectrum of silicone oil in the anterior chamber of a patient obtained with a laser power of $95 \mu \mathrm{W}$ and $1 \mathrm{~s}$ integration time. The spectrum was obtained through an inferior cornea position. Even using this extremely low, eye safe laser power, a clear Raman spectrum of the silicone oil is observed.

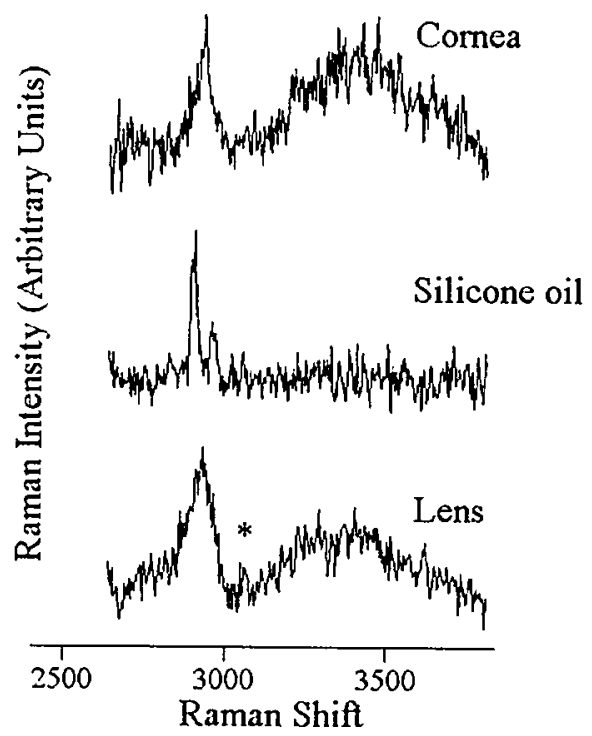

Fig. 4. In vivo Raman spectra of the rabbit eye with the confocal probe positioned to observe the cornea, silicone oil bubble within the aqueous humor, and lens.

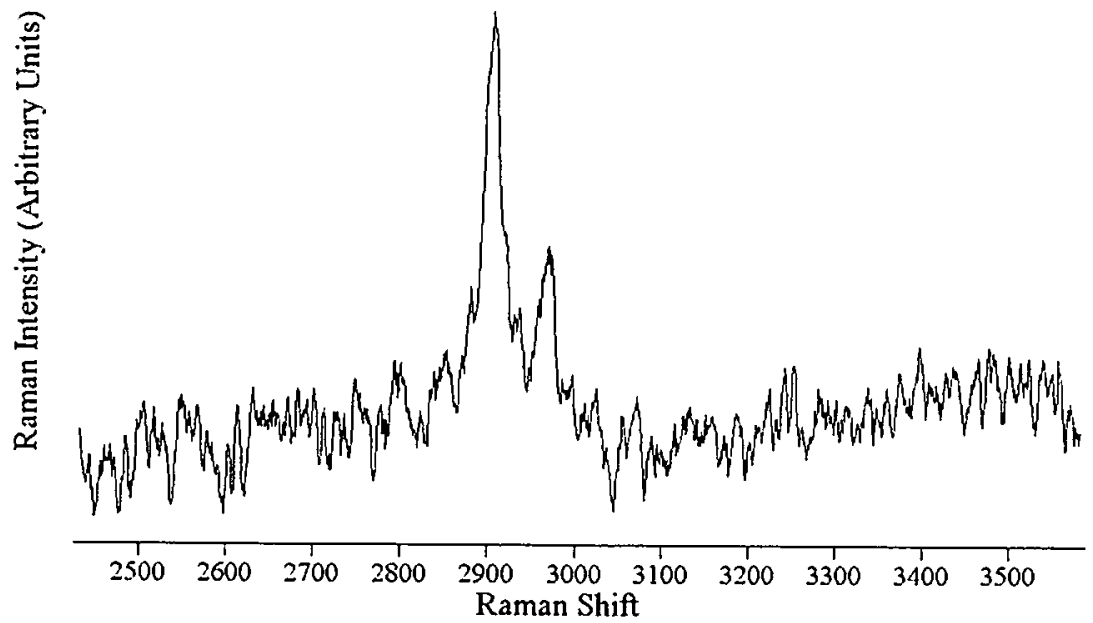

Fig. 5. Silicone oil in anterior chamber of patient. 


\section{Discussion}

The Raman spectroscopic technique allows for detection and determination of silicone oil in the anterior chamber in the living rabbit eye. The Raman spectra of the cornea, aqueous humor or lens are clearly distinguished from the introduced silicone oil. The spectrum obtained from the patient shows the feasibility of detection of the silicone oil with a safe laser power in $1 \mathrm{~s}$. The patient had no discomfort during or after the measurement.

In most cases, the experienced ophthalmologist can reliably inspect the eye for silicone oil with the slitlamp biomicroscope. The normal or healthy eye is an optical transparent medium. If visible light passes through pure water, the water appears transparent. The signal depends on the optical impedance of boundaries resulting in visualization of contours of the optical discontinuities. For instance, a droplet of silicone oil in the aqueous humor may be detected due to its difference in refractive index compared to water on which its reflection coefficient depends. For light, the reflection coefficient is equal to:

$\mathrm{RC}_{\text {light }}=\left[\left(\mathrm{n}_{\text {tissue }}-\mathrm{n}_{\text {water }}\right) /\left(\mathrm{n}_{\text {tissue }}+\mathrm{n}_{\text {water }}\right)\right]^{2}$

where $n$ is the index of refraction.

The reflection coefficient of light gives the intensity of the reflected light rather than the amplitude that cannot be seen or measured directly. As the index of refraction of 5000 centistokes silicone oil is at $25^{\circ} \mathrm{C}$ between 1.403 and 1.405 (enclosed information with AdatoSil $5000^{\mathrm{TM}}$ ) compared to 1.336 for the aqueous humor, about $0.13 \%$ will be reflected. This weak optical boundary will also cause some diffraction that may reveal the contour of a silicone oil droplet at slitlamp examination.

However, if there are opacities that obstruct viewing, ultrasound is an alternative diagnostic technique(30) that can be utilized. Ultrasound is also based upon reflection. In certain circumstances, it can be hindered by ghost images due to acoustical impedance mismatches or acoustical opacities like gas depositions. The ultrasound signal depends on acoustical impedances that are given by other characteristics than optical refraction index discontinuities. Here also boundaries are visualized. For ultrasound, the reflection coefficient is equal to:

$R C_{\text {us }}=\left(\rho c_{\text {tissue }}-\rho c_{\text {water }}\right) /\left(\rho c_{\text {tissue }}+\rho c_{\text {water }}\right)$

where $\rho$ is the density and $c$ is the speed of sound in material. The acoustic impedance of a medium is defined by $\rho c$.

The reflection coefficient of ultrasound gives the pressure (amplitude) of the reflected sound. In reference(31) the $\mathrm{RC}_{\mathrm{us}}$ for silicone oil is compared with the $\mathrm{RC}_{\mathrm{us}}$ for the different eye tissues. For silicone oil $\rho=0.96$ to 0.98 and $c=1000 \mathrm{~m} / \mathrm{s}$, compared to $1500 \mathrm{~m} / \mathrm{s}$ for water. This implies that about $21 \%$ of the sound waves will be reflected. In contrast to the optical slitlamp image that reveals the contour of a droplet due to refraction rather than reflection, the ultrasound image of the silicone oil droplet is formed by its specular reflection.

As we measure the traveling time of the ultrasound pulse, we can visualize the reflection perpendicular to the axis of the probe where with the optical measurements we look along the axis of the probe. In the optical technique by selective illumination with the slit projection, a cross section can also be visualized. Both techniques have in common that we must have 'a priory' knowledge of the substances we are looking for. For instance, if the droplet of silicone oil would be replaced by a droplet of perfluorcarbon, that also exhibits a slight 
optical reflection and a huge acoustic reflection (due to its low propagation velocity(32), we can observe on the ultrasound image the "light house" effect(33-34). This helps to discriminate between silicone oil and perfluorcarbon.

However, to determine the molecular composition of a material, analytical chemistry techniques(35) are required to obtain such information. One analytical technique is Raman spectroscopy that reveals material characteristics by which its structure can be determined. If a probing beam of light, described by an electric field $\mathrm{E}$, is incident on a material, it induces a dipole moment $\mu$ within the molecules of the material:

$\mu=\alpha \times \mathrm{E}$

where $\alpha$ is the polarizability of a molecule. The polarizability is a proportionality constant describing the deformability of the electron cloud of the molecule. This deformation can result from two effects and is expressed as $\alpha=\alpha_{0}+\Delta \alpha$. The first term $\alpha_{0}$, results from the deformation of the electron cloud by an electric field of the light itself and leads to Rayleigh scattering from the molecules. Raman activity results from the second term $\Delta \alpha$, which is much smaller and depends on the molecular bonds with polarizabilities that vary as functions of intramolecular distances. Light interacting with such molecules will be absorbed and re-emitted at a different wavelength (Raman shifted) corresponding to the vibrational frequency of the molecular bond. These vibrational frequencies are specific for the molecules and bonds.

Using Raman spectroscopy, we have introduced another noninvasive technique to detect silicone oil in the eye. In contrast to ultrasound, the Raman spectroscopic technique in ophthalmology is in its very early stages. It is, like ultrasound, a complex technique. However, when developed as a "black box", useful information can easily be obtained. With Raman spectroscopy, no 'a priory' knowledge about the chemical composition of the substance under investigation is required.

This technique can also be applied to investigate the presence of silicone oil in the human eye to detect a pupil block, an obstructed iridectomy and the amount of oil filling in the anterior chamber. Since in this modality no contact is made between probe and cornea, the cornea is spared of mechanical stress. Obtaining Raman spectra through a peripheral part of the cornea has the advantage that a considerable part of the excitation beam is projected on the iris diaphragm rather than on the sensitive retinal tissue. With some adaptation, the probing beam can be directed through the anterior chamber (AC) perpendicular on the visual axis in order to prevent the excitation light of reaching the retina, except for scattered light. Due to the high Raman peak intensity, the exciting laser power could be reduced even more, which may facilitate scanning techniques(36) to detect silicone oil droplets at unknown locations or to investigate the anterior chamber angle. In directing the probing beam through a contact goniolens, silicone oil bubbles or silicone oil impregnation could be detected in the anterior chamber angle, which could help in diagnosing an obstruction of the outflow channels for the aqueous humor by the silicone oil. Furthermore, the increased reflectivity(31) on ultrasound biomicroscopy (UBM) in the AC angle and at the iris, or rarely in the $\mathrm{AC}$ angle alone, could be studied in order to understand the behavior and tissue interaction of the silicone oil.

The use of the Raman technique in the animal model could help in the development and usefulness of new intraocular biomaterials like bioadhesives(37), vitreous substitutes, and devices for the release of pharmacological agents, where the dynamics of drug release or 
influence of intraocular used vitreous substitutes on theses drugs(38) could be investigated. The development of a probe design to obtain Raman spectra from the vitreous humor needs further research efforts. It is conceivable that during a PPV, Raman spectra could be obtained by means of a fiber optic through the intraocular instruments.

\section{Conclusion}

Due to its high specificity and good signal to noise ratio on biomaterials, Raman spectroscopy is a promising diagnostic tool to determine characteristics or functions of IOLs, vitreous substitutes, drug release implants, adhesives, and to develop new applications of biomaterials in the eye.

\section{References}

1. Allan B. Closer to nature: new biomaterials and tissue engineering in ophthalmology. $\mathrm{Br} J$ Ophthalmology 1999;83(11):1235-1240. Review.

2. Ryan S, Ogden T, Hinton D, Schachat A. Retina. Mosby_Year Book St Louis MO USA 2000.

3. Cibis PA. Becker B, Okun E, Canaan S. The use of liquid silicone in retinal detachment surgery. Arch Ophthalmol 1962;68:590-599.

4. Szurman P, Bartz-Schmidt KU. Silicone oils in retinal surgery.(Article in German] Ophthalmologe 2000 Jul;97(7):514-25. Review.

5. Akduman L, Karavellas MP, MacDonald JC, Olk RJ, Freeman WR. Macular translocation with retinotomy and retinal rotation for exudative age-related macular degeneration. Retina 1999;19(5):418-23.

6. Eckardt C. Eckardt U, Conrad HG. Macular rotation with and without counter-rotation of the globe in patients with agerelated macular degeneration. Graefes Arch Clin Exp Ophthalmol 1999 Apr:237(4):313-25.

7. Vander JF. Macular translocation.Curr Opin Ophthalmol 2000 Jun;11(3):159-65. Review.

8. Pertile G. Claes C. Silicone oil vs. gas for the treatment of full-thickness macular hole. Bull Soc Belge Ophthalmol 1999;274:31-36.

9. Margherio AR. Macular hole surgery in 2000. Curr Opin Ophthalmol 2000;11(3):186-90.

10. Irvine AR, Lonn L, Schwartz, D, Zarbin M, Ballesteros F, Kroll S. Retinal detachment in AIDS: long-term results after repair with silicone oil. Br J Ophthalmol 1997;81(3):180-3.

11. Federman JL. Schubert HD. Complications associated with the use of silicone oil in 150 eyes after retina-vitreous surgery. Ophthalmology 1988 Jul;95(7):870-6.

12. Han DP, Lewis H, Lambrou FH Jr, Mieler WF, Hartz A. Mechanisms of intraocular pressure elevation after pars plana vitrectomy. Ophthalmology 1989 Sep;96(9):1357-62.

13. Montanari P. Troiano P, Marangoni P, Pinotti D, Ratiglia R, Miglior Mlst. Glaucoma after vitreo-retinal surgery with silicone oil injection: epidemiologic aspects. Int Ophthalmol 1996-97:20(1-3):29-31.

14. Honavar SG, Goyal M. Maji AB, Sen PK, Naduvilath T, Dandona LVST. Glaucoma after pars plana vitrectomy and silicone oil injection for complicated retinal detachments. Ophthalmology 1999 Jan;106(1):169-76; discussion 177.

15. Henderer JD, Budenz DL, Flynn HW Jr, Schiffman JC. Feuer WJ, Murray TG. Elevated intraocular pressure and hypotony following silicone oil retinal tamponade for complex retinal detachment: incidence and risk factors. Arch Ophthalmol 1999 Feb;117(2):189-95. 
16. Gao RL, Neubauer L. Tang S. Kampik A. Silicone oil in the anterior chamber. Graefes Arch Clin Exp Ophthalmol 1989:227(2):106-9

17. Friberg TR, Guibord NM. Comeal endothelial cell loss after multiple vitreoretinal procedures and the use of silicone oil. Ophthalmic Surg lasers 1999:30(7):528-534.

18. Burk LL. Shields MB, Proia AD, McCuen BW 2d. Intraocular pressure following intravitreal silicone oil injection. Ophthalmic Surg 1988 Aug: 19(8):565-9.

19. Heidenkummer HP. Kampik A. Thierfelder S. Experimental evaluation of in vitro stability of purified polydimethylsiloxanes(silicone oil) in viscosity ranges from 1000 to 5000 centistokes. Retina 1992:12(3 Suppl):S28-32.

20. Raman C, Kirishnan K. A new type of secondary radiation. Nature. 1928:121:105.

21. Carey PR. Raman spectroscopy, the sleeping giant in structural biology, awakes. J Biol Chem 1999:274:26625-8. Review.

22. Mulvaney S, Keating C. Raman Spectroscopy. Anal. Chem. 2000:72:145R-157R. Review.

23. Hanlon EB, Manoharan R, Ko TW. Shafer KE. Motz JT. Fitzmaurice M et al. Prospects for in vivo Raman spectroscopy. Phys Med Biol 2000:45(2):R1-59. Review.

24. Erckens RJ, Jongsma FH, Wicksted JP. Hendrikse F. March WF. Motamedi M. Raman spectroscopy in ophthalmology: From experimental tool to applications in vivo. Review. In Press in Lasers in Medical Science.

25. Jongsma F, Erckens R, Wicksted J, Bauer N, Hendrikse F, March W, Motamedi M. Confocal Raman spectroscopy system for noncontact scanning of ocular tissues: An in vitro study. Optical Engineering 1997:36:3193-3199.

26. Bauer N.J.C., Hendrikse F., March W.F. In vivo confocal Raman spectroscopy of the human comea. Comea 1999;18:483488.

27. Erckens RJ, March WF, Jongsma FH, Wicksted JP, Hendrikse F, Smit EE. Motamedi M. Noninvasive Raman spectroscopic identification of the intraocular lens material in the living human eye. In Press in the Journal of Cataract and Refracive Surgery.

28. ANSI. (1993). American National Standards Institute. Safe use of lasers. In: ANSI standard Z 136.1. Laser Institute of America, Orlando U.S.A. 1993.

29. Bauer NJ. Potential applications of confocal Raman spectroscopy in ophthalmology. Thesis University of Maastricht, the Netherlands 1999. ISBN 9052782636

30. Thijssen JM. The history of ultrasound techniques in ophthalmology. Ultrasound in Med \& Biol 1993;19:599-618.

31. Grigera DE, Zambrano A, Cazón GP, Cavanagh E, Girado SG. Ultrasound biomicroscopy in silicone oil-filled eyes. Retina 2000;20:524-531.

32. Jongsma FH. Liquids with a very low ultrasound propagation velocity. Ultrasonics 1979;Sept:233.

33. Nemeth J, Süveges I. Echographic signs of perfluorodecalin. Am J Ophthalmol 1993;115:679-80.

34. Hasenfratz $G$, De la Torre $M$, Haigis $W$. Evaluation of eyes harboring perfluorocarbon liquid with standardised ophthalmic echography. Ger J Ophthalmol 1994:3:19-21.

35. Skoog D. Leary J. Principles of Instrumental Analysis (Fourth Edition). Saunders College Publishing 1992 Orlando FL.

36. Lerner E. Laser microscopy opens a new dimension. Laser Focus World 2000;December: 141-144.

37. Margalit E, Fujii G, Lai JC, Gupta P, Chen S, Shyu J. Piyathaisere DV, Weiland JD, De Juan E jr, Humayun MS. Bioadhesives for intraocular use. Retina 2000:20:469-477.

38. McGuire DE, McAulife P, Heinemann M, Rahhal F. Efficacy of the ganciclovir implant in the setting of silicone oil vitreous substitute. Retina 2000:20:520-523. 


\section{Chapter 9}

\section{Noninvasive Raman spectroscopic identification of the intraocular lens material in the living human}

eye.

In press in J Cataract Refr Surg.

Roel J. Erckens, M.D.1,2 Wayne F. March, M.D. ${ }^{*}$ Franciscus H. Jongsma, Ph.D.2 James P. Wicksted, Ph.D. ${ }^{3}$ Fred Hendrikse, M.D., Ph.D.2 Eefke E. Smit, M.D. ${ }^{2}$ Massoud Motamedi, Ph.D.1

1 Laser \& Spectroscopy Program of the Center for Biomedical Engineering and the Department of Ophthalmology and Visual Sciences, University of Texas Medical Branch Galveston, Galveston, Texas 77555-0456. U.S.A.

2 Department of Ophthalmology, Academic Hospital Maastricht, Maastricht. The Netherlands.

3 Department of Physics, Center for Sensors \& Sensor Technologies. Oklahoma State University, Stillwater, Oklahoma 74078-3072, U.S.A.

Presented as a poster at the annual meeting of the Association for Research in Vision and Ophthalmology, For Lauderdale, Florida, USA, May 2000. Supported by an Unrestricted Grant from Research to Prevent Blindness, Inc., New York, New York, USA.

* Corresponding author 


\section{Abstract}

Purpose: To develop a safe noninvasive technique for identifying the type of material used for previously implanted intraocular lenses in patients.

Setting: Center for Biomedical Engineering and the Department of Ophthalmology and Visual Sciences, University of Texas Medical Branch, Galveston, Texas, USA

Methods: Raman spectroscopy, which is capable of identifying the chemical composition of material, was utilized to noninvasively identify the type of intraocular lens (IOL) implanted after cataract surgery in the past in nine eyes from six patients (these patients were legally blind due to various eye diseases). Three different types of IOLs were characterized: PMMA lenses (5), acrylic lens (3) and silicone lens (1). Confocal Raman spectroscopy, which allows an adjustable integration depth, was used with a low laser power of $95 \mu \mathrm{W}$ and an exposure time of $1 \mathrm{~s}$.

Results: Distinct spectral peaks associated with each type of IOL were obtained. These include spectra peaks at $2840 \mathrm{~cm}^{-1}, 2946 \mathrm{~cm}^{-1}, 3000 \mathrm{~cm}^{-1}$ for PMMA ; $2917 \mathrm{~cm}^{-1}, 2939 \mathrm{~cm}^{-}$ $1,3055 \mathrm{~cm}^{-1}$ for acrylic and $2900 \mathrm{~cm}^{-1}, 2961 \mathrm{~cm}^{-1}, 3048 \mathrm{~cm}^{-1}$ for silicone. The procedure was well accepted by the patients with no complications.

Conclusions: The specific Raman spectra of the intraocular lenses allowed for practical noninvasive determination of the substance of the implanted lens with the use of a safe light dose for an exposure time of $1 \mathrm{~s}$. 


\section{Introduction}

For about 50 years, artificial intraocular lenses have been used to replace the extracted cataract lens. The first lens made of acrylic material was implanted in 1949 by Ridley(1). Since then, several materials have been tested worldwide for optical performance and biocompatibility while implanted in the human eye. Today the most frequently used lens materials(2,3) for implantation are Polymethylmethacrylate (PMMA), acrylic, hydrogel and silicone.

For management of certain clinical and surgical complications associated with the presence of an intraocular lens (IOL), it is necessary to know the type of lens that has been implanted before the patient can be treated. For example, if the patient needs a pars plana vitrectomy (PPV) with silicone oil, it is mandatory to know what type of lens is in situ. However, that information may not always be available. The silicone oil introduced during the PPV may react with the silicone lens ${ }^{(4-7)}$, causing a loss of visibility in the surgical field of view during the procedure. In other cases, it has been reported that silicone lenses appear to be more vulnerable(8) to a laser posterior capsulotomy than other lens types. In such cases, dark pits are observed on the surface of the IOL following Nd-YAG laser capsulotomy. These laser lesions may resemble pigment deposits that can be found in uveitis.

To determine the type of IOL in vivo, several noninvasive techniques have been investigated in the past. Using magnetic resonance tomography(9), it was possible to obtain the dioptric value of the IOL, but it was impossible to identify the material of the IOL. Recently, optical coherence tomography (OCT) of the anterior segment was studied(10) as a diagnostic and biometric tool. However, despite the excellent results in tissue, it was impossible to visualize an IOL in a patient due to the homogeneous and translucent structures of these lenses.

Applications of Raman Spectroscopy for noninvasive assessment of biochemical structures of tissues have been reported by various groups(11-14). We have developed and tested a long working distance confocal Raman system for ophthalmic applications(15.16). The confocal nature of this setup allows the integration depth, by which the Raman scattered light is collected, to be changed (by varying the diameter of the collection fiber(15)). In an in vivo application, this means that the Raman signal is collected only from the IOL, while signals from other ocular tissue, such as the aqueous humor and the vitreous humor, will be excluded. In a recent study, we have utilized this confocal setup to determine the Raman spectra from several intraocular lens materials in vitro(17). Distinctive Raman signatures were observed from four different materials: silicone, polymethylmethacrylate (PMMA), acrylic, and hydrogel. This previous study investigated the Raman spectra from these materials over two spectral ranges, with the most intense peaks being observed over the spectral range from $2633.8 \mathrm{~cm}^{-1}$ to $3819.6 \mathrm{~cm}^{-1}$, while utilizing two different laser powers, $25 \mathrm{~mW}$ and $1 \mathrm{~mW}$. In this study, we have further modified this technique so that lower laser powers can be used to establish noninvasively the type of an implanted lens from a human patient. Raman spectroscopy, combined with the earlier developed confocal probe(15) and a laser wavelength of $514.5 \mathrm{~nm}$ with a laser power of $95 \mu \mathrm{W}$ and an exposure time of $1 \mathrm{~s}$, was used to obtain the Raman spectra of the lenses. In the current study three different eye lenses were characterized spectroscopically: PMMA, acrylic and silicone. 


\section{Materials and Methods}

The confocal Raman system is shown in Figure 1 and is described in our previous work(15). The Raman system consists of a long working distance $(11 \mathrm{~mm})$ microscope objective (entrance lens) with a high light-gathering power $(\mathrm{NA}=0.5)$ to deliver the probing light beam, as well as to collect the Raman backscattered light, and a holographic beamsplitter that guides the Raman scattered light into a large aperture lens by which the Raman scattered light is focused on a fiber. The diameter of the probing beam at the focal plane was estimated to be $6 \mu \mathrm{m}$, thus larger than expected from the diffraction limited entrance lens. This is due to the optical noise introduced by the holographic beamsplitter in the collimated laser beam. The entrance lens was mounted on a micropositioner for accurate focusing in the IOL in the subject's eye. The collecting fiber that acts as a pinhole in the confocal system is connected to the spectrometer equipped with a liquid nitrogen cooled CCD camera. By choosing the diameter of the fiber, the integration depth within the sample can be set. In this study, a $400 \mu \mathrm{m}$ fiber was used yielding a sample integration depth of about $1 \mathrm{~mm}$. The laser source was an argon laser emitting at $514.5 \mathrm{~nm}$. The probing laser power of $95 \mu \mathrm{W}$ and exposure time of $1 \mathrm{~s}$ (i.e. energy of $95 \mu \mathrm{J}$ ) were used throughout this study. Using an optical density filter, the beam was attenuated to about $14 \mu \mathrm{W}$ for aligning purposes and initial focusing on the IOL of the patient.

We have improved our signal-to-noise ratio by a factor of two by increasing the slit setting on our spectrometer from 200 microns, which had been used in our in vitro study(17), to 400 microns. Although this decreases the spectral resolution of our instrument by about $33 \%$,

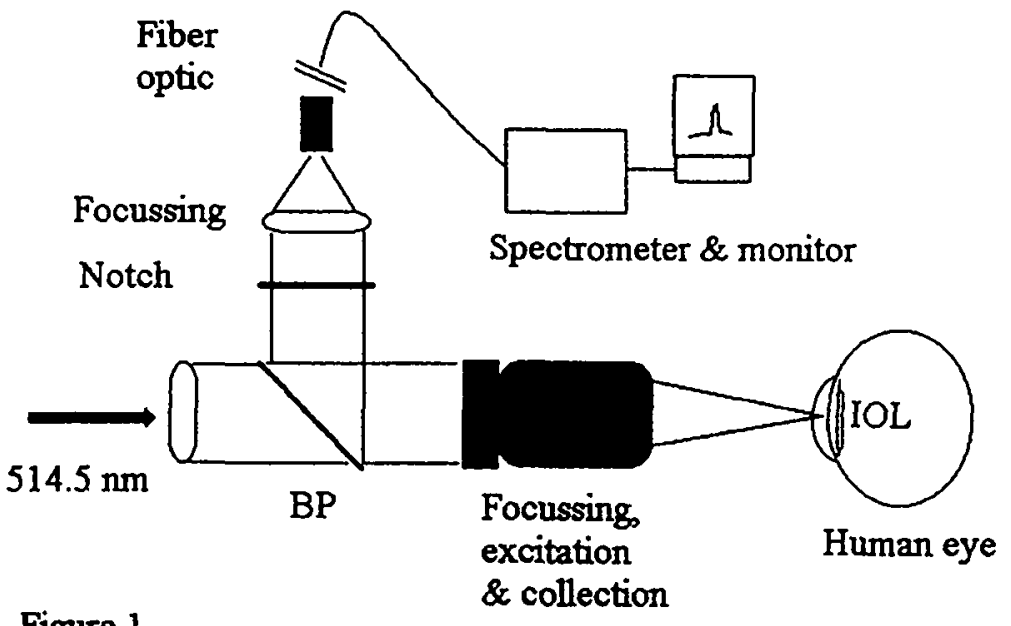

Figure 1

Figure 1. Schematic diagram of the confocal Raman system used in this study.

1-laser beam; 2\&3-collimator; 4-holographic bandpass filter transmitting the excitation light and reflecting the Raman shifted light; 5-long distance microscope objective; 6-eye; 7-notch filter; 8high speed lens; 9- fiber acting as a confocal pinhole; 10-fiber towards spectrometer. 
the increased signal-to-noise enabled the in vivo measurements in this study using an incident laser power of $95 \mu \mathrm{W}$. This should be compared to the $1 \mathrm{~mW}$ powers that were utilized in our previous in vitro study(17).

For proper positioning of the patient, a modified chin rest and forehead holder from a slit lamp were used and mounted on an optical bench to support the head of the patient. This allowed for height control and made the setup accessible for all types of patients. Six legally blind patients, ranging in age from 46 to 76 years old, were selected for this study. From these six patients, three patients had a fellow eye that was not legally blind, and therefore, no spectrum was obtained from this eye. These patients were legally blind due to age-related macular degeneration (AMD), proliferative diabetic retinopathy (PDR) or glaucoma. The study was performed under the approval of the Institutional Review Board. All patients gave Informed Consent after the study protocol was explained to them. Complete details of the patients and types of IOLs are listed in Table 1. All patients were asked to fixate with the fellow eye on an illuminated target in the distance. The luminosity of the target was adjusted to the patient's visual acuity in this eye. Except for patient 4, who had an anterior chamber lens, the pupil of the eye under investigation was dilated 30 minutes before the actual measurements using mydriatic eye drops (Mydfrin and Mydriacyl, Alcon). After the patient was properly positioned at the modified slit lamp holder, the initial focusing was carried out as follows: with the exciting light switched off, the entrance lens was positioned about $10 \mathrm{~mm}$ from the patient's cornea. Next, the attenuator (optical density filter) was placed in the exciting beam path and the laser light was turned on. By moving the entrance lens toward the cornea, the IOL was detected, typically after 4 to 6 Raman scans. When the IOL was localized, the attenuator was moved aside and a regular Raman scan was collected.

To obtain the means of calibration for the Raman intensities in the in vivo setup, a Raman signal from a PMMA IOL (Pharmacia 812 B) was obtained in vitro. Since the IOL is in an aqueous environment while implanted in the eye, we simulated this condition by placing a PMMA lens in sterile water placed in a cuvette. The experimental settings of laser power and exposure time similar to the patient study were used to obtain the Raman spectra of IOL and water.

Table 1. Details of IOLs in patients. NO=Not obtained; $P C=$ Posterior Chamber; $A C=$ Anterior Chamber

$\begin{array}{lllll}\text { Patient } & \text { Eye } & \text { Material } & \text { Position } & \text { Model and manufacturer } \\ 1 & \text { OD } & \text { NO } & & \\ & \text { OS } & \text { PMMA } & \text { PC } & \text { Pharmacia 812 B, Pharmacia } \\ 2 & \text { OD } & \text { Acrylic } & \text { PC } & \text { AcrySof MA60BM, Alcon } \\ & \text { OS } & \text { Acrylic } & \text { PC } & \text { AcrySof MA60BM, Alcon } \\ 3 & \text { OD } & \text { Acrylic } & \text { PC } & \text { AcrySof MA60BM, Alcon } \\ 4 & \text { OS } & \text { PMMA } & \text { PC } & \text { CilcoCZ70BD, Alcon } \\ 4 & \text { OD } & \text { NO } & & \\ 5 & \text { OS } & \text { PMMA } & \text { AC } & \text { Pharmacia UV65B, Pharmacia } \\ 5 & \text { OD } & \text { PMMA } & \text { PC } & \text { Pharmacia 812 B, Pharmacia } \\ & \text { OS } & \text { NO } & & \\ 6 & \text { OD } & \text { Silicone } & \text { PC } & \text { Foldable 920, Pharmacia\&Upjohn } \\ & \text { OS } & \text { PMMA } & \text { PC } & \text { Pharmacia 815 HS, Pharmacia }\end{array}$




\section{Results}

Raman spectra contain specific details about the chemical composition of the material that is being probed. Previously, we have reported the spectral features for four different IOL lens materials in both the lower, as well as the higher region of the spectra(17). In the current study, we observed that most of the unique Raman spectral features for three types of lenses were located in the higher scan region of the spectra in the range of 2800 and $3100 \mathrm{~cm}^{-1}$. Figure 2 shows the spectra in vivo from three different patients, each patient having a different lens type. The $\mathrm{x}$-axis depicts the Raman shift in wavenumbers $\left(\mathrm{cm}^{-1}\right)$, and the $y$-axis depicts the intensity in arbitrary units. Although raw data will provide sufficient information for identification of the type of IOL in real-time, the spectra as presented here were baseline corrected and smoothed using a software program (Grams/386, Galactic Industries Corp.). Specifically, PMMA exhibited a large peak at $2946 \mathrm{~cm}^{-1}$ with a small peak at each shoulder at $2840 \mathrm{~cm}^{-1}$ and $3000 \mathrm{~cm}^{-1}$, while the acrylic IOL showed a broader structure with two peaks located at $2917 \mathrm{~cm}^{-1}$ and $2939 \mathrm{~cm}^{-1}$ and another peak located at $3055 \mathrm{~cm}^{-1}$. In the silicone IOL, the presence of a large peak located at $2900 \mathrm{~cm}-1$, accompanied by another peak with about half the amplitude of the $2900 \mathrm{~cm}^{-1}$ peak located at $2961 \mathrm{~cm}^{-1}$, and a smaller peak located at $3048 \mathrm{~cm}^{-1}$ was observed. These lenses do not contain much water as evidenced by the low intensity of the spectra around $3390 \mathrm{~cm}^{-1}$, which is indicative of $\mathrm{OH}$ bonds and can be used to quantify the amount of water in tissue (see Figure 3 where the water Raman spectrum is indicated by the dashed line). The intensity axis ( $y$-axis) in Figure 2 is not given in absolute values to allow easy comparison of the relative peak positions. Laser power $95 \mu \mathrm{W}$, integration time $1 \mathrm{~s}$.

Figure 3 shows a Raman spectrum of PMMA IOL (continuous line) that is submersed in a cuvette filled with sterile water. This figure also displays the Raman spectrum of water (dashed line). The absolute intensity scale in this figure allows a comparison of the relative peak intensities of the PMMA IOL and the water Raman spectra. Experimental conditions used to obtain these spectra were similar to those used in the patient study. As demonstrated here, the strength of the Raman signal originated from water is significantly less than that coming from the IOL. Therefore, little or no interference from the aqueous solution sur-

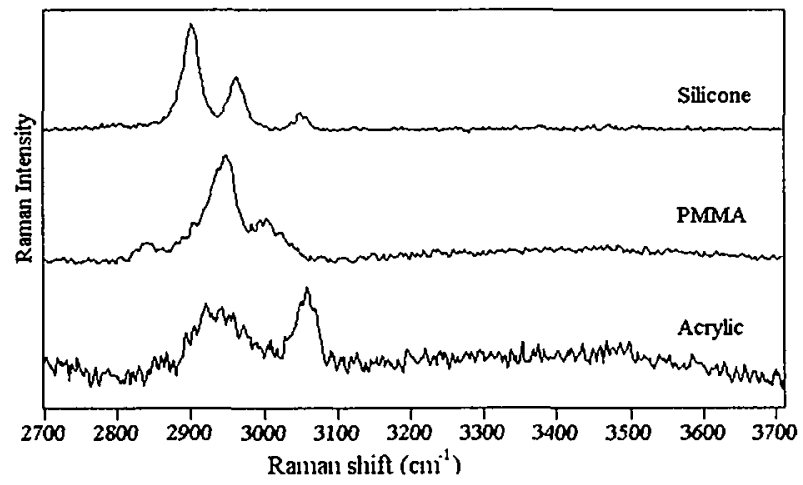

Figure 2. Raman spectra of PMMA, acrylic and silicone lenses obtained in vivo from three patients. 
rounding the IOL is expected when Raman spectroscopy is used for in vivo identification of an IOL. This effect facilitates the ease of focusing a laser beam on the IOL.

All the procedures performed during this pilot study were uneventful. The patients did not complain of any discomfort or complication while the short diagnostic procedure was performed. No side effects during or after the data collection were observed by the investigators.

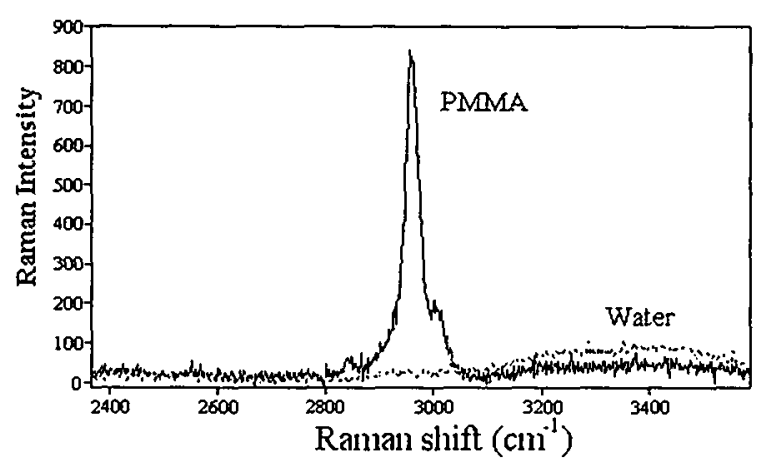

Figure 3. Raman spectrum of water and of a PMMA IOL obtained in vitro in a cuvette. Continuous line: PMMA lens. Dashed line: Water.

\section{Discussion}

In vivo applications of Raman spectroscopy offers great potential for noninvasive assessment of ocular tissue and in vivo optical sectioning of the eye. For the first time, we have demonstrated that it is possible to noninvasively identify the material that is used in IOL by collecting a Raman spectrum of the lens for one second, while using a safe light dose. Each of the nine IOLs listed in Table 1 from the six patients used in this study was correctly identified using our confocal Raman system. As the spectra are available in real-time for interpretation, this method proves to be a fast and patient friendly diagnostic tool to obtain information of the composition of an implanted IOL. The results show a clear difference between the Raman spectra of various intraocular lenses. Considering the previously published data(18.19), Raman signals arising from ocular tissues are much weaker, and they will not interfere with the detection of the IOLs since the intensities of the Raman spectra originating from artificial lenses are much stronger than those originating from ocular tissue. These findings are consistent with previous studies performed in vitro(17). Furthermore, as we have previously described, the confocal approach limits the interference of undesired Raman spectra arising from the substances surrounding the integration volume such as the aqueous humor, which is mainly composed of water. The typical Raman spectral features in the longer spectral region provide the information for the discrimination between the lenses. However, the lower spectral region(17) can also be used or combined with the longer spectral region to interpret the data in case of interference from other substances like deposits on the IOL. 
From a safety point of view, the laser beam focused in the IOL projects a spot size of about $1.5 \mathrm{~cm}^{2}$ on the retina. According to the ANSI standard(20), a $95 \mu \mathrm{W}$ laser beam from an argon laser operating at $514.5 \mathrm{~nm}$ delivered over a $1 \mathrm{~cm}^{2}$ can be used for 15 seconds continuously. Thus, the light dose used in our study is 15 times lower than the ANSI damage threshold for the retina since our exposure time was only one second. Further, it should be noted that the laser power used to obtain the Raman spectra in this study is on average considerably less than what is used during an eye examination with an indirect ophthalmoscope(21). A previous Raman study on the biocompatibility of PMMA lenses implanted in rabbit eyes indicated that the material did not change its structure after implantation(22). This was determined based on the level of monomeric methyl methacrylate, which remained constant after the PMMA lens was implanted. Similar studies can be conducted on current and new IOL materials. However, the eye safety issue was not addressed in this previous study(22). Further applications of this technique could potentially be developed including characterization of implantable contact lenses for high myopia(23) (phakic lens implantation). Contact between IOL and phakic lens may have undesirable side effects like cataract formation in the phakic lens. Thus, confocal Raman spectroscopy could be used to discriminate between IOL and phakic lens and estimate the distance between these lenses since each lens has unique Raman spectra.

Other applications include the use of the confocal Raman setup to characterize and assess IOL coatings such as Teflon AF that has been shown ${ }^{(24)}$ to display a distinctive Raman spectrum and has been used to coat IOLs to obtain an adhesive effect to increase the biocompatibility of IOLs. The Raman technique described here could also be used to monitor in vivo the changes or deposits(25) on the IOLs. Furthermore, leakages in filling capsular bags after silicone oil injection in animal research in the development of artificial lenses that can accommodate(26) could be monitored, as well as the removal of silicone oil from lens surfaces with new solvents(27.28).

\section{Conclusion}

In summary, the results of this study indicate that confocal Raman spectroscopy can be used as an eye-safe, noninvasive diagnostic technique to obtain information about the chemical composition of the implants that are used in patients.

\section{References}

1. Ridley NHL. Intraccular acrylic lenses. Trans Ophthalmol Soc UK 1951; LXXI: 617- 621.

2. Cataract surgery, the state of the art. James P. Gills. 1998. Slack Inc., Thorofare, NJ.

3. Chehade M, Elder M. Intraocular lens materials and styles: a review. Aust N J Z Ophthalmol 1997; 25(4):255-63.

4. Bartz-Schmidt KU, Konen W, Esser P, Walther P, Heimann K. Intraokulare Silikonlinsen und Silikonöl. Klin. Monatsbl. Augenheilkd. 1995; 207:162-166.

5. Kusaka S, Kodama T, Ohashi Y. Condensation of silicone oil on the posterior surface of a silicone intraocular lens during vitrectomy. Am J Ophthalmol 1996 May: 121(5): 574-5. 
6. Apple DJ, Federman JL, Krolicki TJ, et al. Irreversible silicone oil adhesion to silicone intraocular lenses. A clinicopathologic analysis. Ophthalmology 1996 Oct; 103(10):1555-61: discussion 1561-2.

7. Senn P, Schmid MK. Schipper I, Hendrickson P. Interaction between silicone oil and silicone intraocular lenses: an in vitro study. Ophthalmic Surg Lasers 1997 Sep; 28(9):776-9

8. Newland TJ, McDermott ML, Eliott D. et al. Experimental neodymium:YAG laser damage to acrylic, poly(methyl methacrylate), and silicone intraocular lens materials. J. Cataract Refract Surg 1999 Jan; 25(1):72-76.

9. Tosch U. Bleckmann H, Kaczmarek U. Magnetic resonance tomography diagnosis of the posterior chamber lens. Aktuelle Radiol 1994: 4(5):218-21.

10. Hoerauf H. Wirbelauer C. Scholz C, et al. Slit-lamp-adapted optical coherence tomography of the anterior segment. Graefe's Arch Clin Exp Ophthalmol 2000; 238:8-18.

11. Carey P. Biomedical applications of Raman and Resonance Raman Spectroscopies. 1982. Academic Press.

12. Tu A. Raman spectroscopy in biology. 1984. John Wiley and Sons.

13. Gerrard D. Raman Spectroscopy. [Review] Analytical Chemistry 1994: 66(12):547R-557R.

14. Lyon A, Keating C, Fox A. et al. Raman Spectroscopy. [Review] Analytical Chemistry 1998: 70(12):341R-361R.

15. Jongsma. F. H. M., Erckens. R. J., Wicksted. J. P. et al. A confocal Raman spectroscopy system for non-contact scanning of ocular tissues: An in vitro study. Optical Engineering 1997; 36:3193-3199.

16. Bauer N., Wicksted J., Jongsma F., March W., Hendrikse F. and Motamedi M. Noninvasive assessment of the hydration gradient across the comea using confocal Raman spectroscopy. Invest. Ophthalmol. Vis. Sci. 1998; 39:831-835.

17. Smit E., Erckens R, Hendrikse F, Motamedi M, Wicksted J, March W. Identification of intraocular lens materials using confocal Raman spectroscopy. J. Cataract Refract Surg 1999 Nov: 25(11): 1498-1504.

18. Erckens. R. , Motamedi. M. March. W. and Wicksted, J. Raman spectroscopy for noninvasive characterization of $\propto$ cular tissue: Potential for detection of biological molecules. J. Raman Spectroscopy 1997; 28:293-299.

19. Bauer N.J.C. Potential applications of Confocal Raman spectroscopy in Ophthalmology. Thesis 1999. University of Maastricht, the Netherlands, 1999.

20. ANSI. (1993). American National Standards Institute. Safe use of lasers. In: ANSI standard Z I36.1. Laser Institute of America, Orlando.

21. Bradnam M. Montgomery D, Moseley H, Dutton N. Quantitative assessment of the blue-light hazard during indirect ophthalmoscopy and the increase in the 'safe' operating period achieved using a yellow lens. Ophthalmology 1995; 102(5):799-804.

22. Bertoluzza A, Fagnano C, Monit P. Semerano G, Garcia-Ramos JV, Caramazza R, Cellini M. Raman spectra of intraocular lenses before and after implantation in relation to their biocompatibility. J. Raman Spectroscopy 1987; 18:151-152.

23. Fyodorov S, Zuyev V. Aznabayev B. Intraocular correction of high myopia with negative posterior chamber lens. Ophthalmosurgery 1991; 3:57-58.

24. Legeais J, Werner L. Legeay G, Briat B, Renard G. In vivo study of a fluotocarbon polymer-coated intraocular lens in a rabbit model. J Cataract Refract Surg 1998: 24(3):371-9.

25. Hollick E, Spalton D, Ursell P. Pande M. Biocompatibility of poly(methylmethacrylate), silicone, and Acrysof intraocular lenses: Randomized comparison of the cellular reaction on the anterior lens surface. J Cataract Refract Surg 1998: 24:361366.

26. Nishi O, Nishi K, Mano C, Ichihara M. Honda T. Lens refilling with injectable silicone in rabbit eyes. J Cataract Refract Surg 1998 Jul :24(7):975-982.

27. Hoerauf H, Menz DH, Dresp J. Laqua H. Klin Monatsbl Augenheilkd. O44-a solvent for silicone oil adhesions on intraocular lenses. $1999 \mathrm{Feb} ; 214(2): 71-76$.

28. Langefeld S, Kirchhof B, Meinert H, Roy T, Aretz A. Schrage NF. A new way of removing silicone oil from the surface of silicone intraocular lenses. Graefes Arch Clin Exp Ophthalmol 1999 Mar ;237(3):201-206. 


\section{Summary and Conclusions}

The diagnosis of pathologic conditions in the eye requires the use of chemical and histological techniques. However, these techniques can only be performed in vitro, which makes invasive procedures necessary to obtain the desired material from the eye. Spectroscopy is a common used analytical technique to investigate molecules and tissues in the laboratory. Raman spectroscopy is an excellent technique to investigate biological materials in aqueous environments. In 1928, C.V. Raman discovered the phenomena that if monochromatic light interacts with matter, a small amount of the scattered light is shifted in wavelength. The Raman shifted light corresponds with the vibrational frequency of the molecular bond. Since the Raman effect is weak, only $10^{-6}$ to $10^{-8}$ photons are shifted, a strong monochromatic light source is mandatory. The discovery of the laser in 1960, initiated a tremendous increase in the use of Raman spectroscopy in general. However, a laser is a hazardous light source for the eye since the laser beam is focused by the eye to a small spot size on the retina. This can result in irreversible damage to the retina tissue. The Raman spectroscopic technique offers interesting analytical diagnostic modalities, though the technique requires the use of a potential hazardous light sources for the eye.

This thesis describes the development of the Raman spectroscopic technique into a useful tool for obtaining information on in vivo eye tissues. Since little was done to achieve this goal in the past, the start of the research was in the sample chamber of the spectrometer, next using a commercial probe, towards the final customized design of a safe probe that can be used on a human eye.

Chapter 1 gives an introduction on eye anatomy, physiology and encountered diseases or problems in the ophthalmology department. Furthermore an introduction on general spectroscopic techniques is provided, with an emphasis on Raman spectroscopy. Finally, the aim of the study is discussed.

Chapter 2 provides a general review on the use of the Raman spectroscopic technique in general, while a detailed discussion on the use of Raman spectroscopy for ophthalmic purposes is given. Most articles are in vitro applications determining the protein contents and protein conformation in lenses; and investigation of retina tissue and establish the critical steps in vision like isomerization and determining different chromophores.

Chapter 3 begins with a discussion on the investigation on aqueous samples in vitro obtained in the sample chamber of the spectrometer to test the system performance and characterize several important biomedical molecules. The results of these experiments, together with other findings in literature, encouraged proceeding with further exploration and pursuing this thesis.

Chapter 4 discusses the use of a commercial available Raman probe. Spectra were obtained from different solutions and excised corneas. The experiments performed, made clear that the light dose needed for a legible Raman spectrum was far too high for in vivo applications. These findings made clear that a new probe design was necessary to obtain a high light gathering power and the possibility of optical sectioning. Furthermore, dimensions and setup of the probe should allow for an easy and safe alignment procedure to the animal or human eye. The 'quest' for such a design leads eventually to the development of a confocal probe.

Chapter 5 discusses this confocal probe design in detail. Main features of the probe are: A working distance of about $12 \mathrm{~mm}$, a high light gathering power with a diffraction limited 
outstanding optical quality. This configuration allows enough space between comea and lens to be tolerated by a patient. The integration depth in the tissue can be chosen by means of the diameter of the collecting fiber, i.e. a fiber of $50 \mu \mathrm{m}$ would yield an integration depth of about $130 \mu \mathrm{m}$ in the tissue. The cornea, aqueous humor, and lens could clearly be resolved. Axial scans with this probe show the Raman spectra in a in situ rabbit eye.

Chapter 6 shows that with the confocal probe it was feasible to study cornea hydration in the living cornea of the rabbit. The hydration ratio (water/protein or $\mathrm{OH} / \mathrm{CH}$ ratio) provided the information on the hydration status, as well in physiological conditions as after dehydration the cornea with a dehydrating agent.

Chapter 7 shows the feasibility to study lens hydration in the living rabbit. Using the $\mathrm{OH} / \mathrm{CH}$ ratio, the change in hydration in different parts of the lens can be observed. Due to enhancements in methods and techniques, the light dose could be reduced. The $25 \mathrm{~mW}$ laser power as used in chapter 5 in $1 \mathrm{~s}$ could be reduced to $1 \mathrm{~mW}$ on $0.1 \mathrm{~s}$. This brings the Raman technique in the safe light dose region for use in the eye.

In chapter 8 and 9, the ultimate goal of this thesis is discussed, namely clinical relevant applications of the Raman technique in patients. This goal was reached by using a safe light dose of $95 \mu \mathrm{W}$, and a short data collection time of $1 \mathrm{~s}$. The data collection was well tolerated by the patients and they didn't encounter any discomfort during or after the measurements.

Chapter 8 discusses the detection of intraocular silicone oil in the anterior chamber of the eye of a patient with an opaque comea. It was feasible to detect minute silicone remnants in the anterior chamber of the living rabbit eye.

Chapter 9 discusses the determination of the type of implanted artificial intraocular lens in patients with the Raman technique.

\section{Conclusions}

To adapt the accurate and precise Raman spectroscopic technique to a tool for obtaining in vivo information without interfering with the integrity of the eye tissue, an optical probe with a high light gathering power and high optical quality turned out to be an indispensable part of the setup. Commercially available probes often lack both qualities. Therefore considerable effort has been made to come from commercially available industrial probes, to probes for use in patients. The restricted maximum permissible light dose on the retinal tissue, demand that the Raman shifted photons are collected with a high efficiency. This in combination with the requirement for an optical dissection technique for control of position in the intact eye, led to a confocal approach. It was possible to obtain in the living rabbit eye Raman data on the amount of cornea hydration, determination of silicone oil in the aqueous humor, and the amount of lens hydration. The validation of the confocal setup proved that Raman spectroscopy was useful in obtaining noninvasive information on molecular properties of intraocular structures in patients, which was the aim of this thesis. The clinical use of the current probe is limited but it is a first step towards further development of this noninvasive technique for ophthalmic use and hopefully establishing the diagnostic use in ophthalmic diseases. 


\section{Future}

The ophthalmic Raman spectroscopic setup can be seen as a chain of which some links, such as the spectrometer and the light source, cannot be optimized much. Whereas other links, such as the type of Raman spectroscopy or the configuration of the optical probe, earns closer examination. The spectrometer in this study had a focal length of $0.5 \mathrm{~m}$ giving adequate resolution, while the power of the argon laser $(5 \mathrm{~W})$ exceeded by far the safe dose to be delivered in a short time. This high output was strongly decreased to obtain safe levels for intraocular use. However, other Raman spectroscopic techniques might improve the sensitivity and the accuracy considerably. The use of 2-wavelength excitation provides interest since in this way the contribution of fluorescence to the Raman signal can be minimized afterwards. In case of a specific substance with a marked Raman peak position, resonance Raman could be applied to increase the intensity of that specific Raman signal. The quantum efficiency of the current CCD detectors in the wavelength up to $700 \mathrm{~nm}$ is reaching the physical limits. Development of detectors with increased quantum efficiency at the near infrared could be of interest, though the Raman intensity is inversely dependent on the wavelength used for excitation. Consequently, this would imply that more laser power is used and/or longer integration times. For patient comfort, an integration of seconds is preferable. An advantage of the use of longer wavelengths is, that they pose a lesser harmful threat to the retina tissue. So there will always be the trade off between excitation source, detector and integration time. However, further refinement on probe design offers some interesting possibilities.

The confocal probe works in a noncontact fashion. Due to the index of refraction of the eye (tear film $n=1.37$ ) and the surrounding air $(n=1.00)$, it is very difficult to probe across the anterior chamber of the eye. The probing beam will be diffracted towards the retina, unless an extreme position at the side of the eye is used. For this reason a probe design that eliminates the index of refraction mismatch by means of a contact lens approach could perform well. In ophthalmology the use of contact lenses for either diagnostic or therapeutic purposes is daily routine. So a modified probe design preferably at 90 degrees, could study aqueous humor contents allowing higher excitation energies without compromising the retina.

Several substances in the aqueous humor are of interest to investigate: For instance, phenylalanine is a molecule of importance since in the disease phenylketonurea, the elevated level could be detected and being able to measure the concentration could help in regulating the patients diet. Other substances of interest in the aqueous humor include lactate, urea, glucose, and amino acids. Also pharmacokinetics, transport and bioavailibility of pharmacological substances could be studied. Ultimately, measurements throughout the vitreous cavity shall be of utmost value since the vitreous humor plays a key role in physiology and disease of the eye.

Another promising tool for application with Raman spectroscopy is a Multivariate Statistical Method to detect different chemical substances dissolved in the same sample (e.g. different solvents like glucose, lactate, urea, and amino acids in the aqueous humor). So Multivariate Statistical Methods can be utilized to extract the correct information if analysis has to be made of a large number of parameters for the object under investigation, and if 
there is overlap of different Raman spectral features or if spectra are 'buried' in larger interfering structures.

Given the mentioned opportunities and the rapid development of new Raman techniques, it can be expected that Raman spectroscopy in the near future will play a significant role in helping the ophthalmologist to obtain the desired information of different eye tissues in a patient friendly modality. 


\section{Samenvatting en conclusies}

Voor de diagnostiek van pathologische processen in het oog zijn chemische en histologische bepalingen van groot belang. Echter deze bepalingen kunnen alleen in vitro verricht worden, waarmee dus invasieve handelingen in het oog nodig zijn om het vereiste materiaal te verkrijgen. Een veel gebruikte laboratorium techniek is de spectroscopie. Voor de meting van biologische (waterhoudende) weefsels is Raman spectroscopie een nauwkeurige en betrouwbare techniek. Deze techniek werd door C.V. Raman ontwikkeld na zijn ontdekking in 1928 dat monochromatisch licht voor een (zeer) klein deel van kleur veranderd bij het passeren van een vloeistof. De kleurverschuiving die optreedt hangt af van de trillingstoestand van de moleculen waaruit de vloeistof bestaat en is zó karakteristiek, dat het Raman spectrum representatief is voor het betreffende molecuul. Omdat slechts ongeveer $10^{-6}$ tot $10^{-8}$ fotonen (lichtdeeltjes) een Raman verschuiving vertonen, is een sterke monochromatische lichtbron vereist. Sinds de ontdekking van de laser in 1960 kan hieraan goed voldaan worden. Voor het oog echter, is een laser een potentieel gevaarlijke lichtbron, omdat de evenwijdige bundel zich door het oog tot een klein spotje op het netvlies laat focusseren. Het oog werkt als een brandglas waarbij irreversibele schade aan het netvlies kan ontstaan. Zelfs laser pointers met een vermogen van enkele milliWatts worden als gevaarlijk beschouwd. Met de Raman spectroscopie beschikken we dus over een nauwkeurige techniek voor chemische diagnostiek maar tevens is het toepassen van deze techniek juist voor het oog potentieel buitengewoon riskant.

Het doel van dit onderzoek was dan ook om een methode te ontwikkelen om Raman spectroscopie in het levende oog toe te passen zonder dat het oog gevaar loopt. Bij de start van het proefschrift werd een praktisch onontgonnen onderzoeksgebied aangetroffen. Daarom werd de Raman techniek eerst getest in de monsterkamer van een Raman spectrometer. Daarna werd met een commerciële Raman sensor ("probe") naar oplossingen in een cuvette gekeken. Tenslotte werd een opstelling gebouwd die het uiteindelijk mogelijk maakte om veilig in het menselijk oog te meten.

In hoofdstuk 1 wordt een introductie gegeven in de anatomie, de fysiologie en de pathofysiologie van het oog en de diagnostische mogelijkheden en problemen in de oogheelkunde. Tevens wordt hier de spectroscopische techniek geintroduceerd met speciale aandacht voor de Raman spectroscopie. Tenslotte wordt het doel van het onderzoek beschreven.

In hoofdstuk 2 wordt een algemeen overzicht gepresenteerd over het gebruik van Raman spectroscopische technieken terwijl een zeer gedetailleerd overzicht wordt gegeven van de wetenschappelijke literatuur op het gebied van de toepassing van Raman spectroscopie in de oogheelkunde. Hierbij is gestreefd naar volledigheid in het citeren van de auteurs. Tevens worden op dit gebied enkele bijzondere octrooien genoemd. De meeste artikelen beschrijven in vitro toepassingen waarin de proteinen en proteïne configuratie in lenzen aan bod komen. Ook retina weefsel is onderzocht alsmede de kritische biochemische stappen (op retinaal niveau) die een rol spelen bij het tot stand komen van de visuele impuls, zoals isomerisatie en de functies van de verschillende chromoforen.

Hoofdstuk 3 begint met het onderzoek van in vitro verkregen waterige oplossingen in de monsterkamer van de spectrometer om de potentie van de Raman techniek betreffende het karakteriseren van verschillende biomedische moleculen die in de oogheelkunde relevant zijn te testen. Ook is onderzocht in hoeverre de natuurlijke fluorescentie, die de kwaliteit 
van het Raman signaal sterk beïnvloed, kan worden onderdrukt door gebruik te maken van langere golflengten. De resultaten van deze experimenten, samen met de informatie uit de literatuur, motiveerden verder onderzoek.

In hoofdstuk 4 worden de resultaten beschreven die bereikt kunnen worden met een commercieel beschikbare probe. Met deze probe werden spectra verkregen van verschillende oplossingen en van getrepaneerde corneas. Deze experimenten toonden duidelijk aan dat de hoeveelheid licht die nodig was om een betrouwbaar Raman spectrum te verkrijgen veel te hoog was voor in vivo toepassingen. Ook de ruimtelijke selectiviteit schoot anzienlijk tekort. Dit leidde tot de conclusie dat een nieuw optisch ontwerp nodig was om een systeem te verkrijgen met een grote lichtsterkte en de mogelijkheid om optische coupes te maken. Additionele eisen aan de probe waren dat de afmetingen en de uitvoering een veilige en eenvoudige uitlijn procedure mogelijk maakten. Dit laatste is van eminent belang bij in vivo metingen.

De "zoektocht" voor dit ontwerp leidde uiteindelijk tot de ontwikkeling van een confocale probe.

In hoofdstuk 5 wordt een gedetailleerde beschrijving van de confocale probe gegeven. De belangrijkste eigenschappen van de probe zijn dat deze een vrije werkafstand in lucht van $12 \mathrm{~mm}$ heeft en dat hij zeer lichtsterk is bij een hoge optische kwaliteit. De hoge optische kwaliteit is bereikt door een microscoop objectief met een lange werkafstand te nemen waarmee de probe buigingsbegrensd is. In het oog wordt de werkafstand door de brekingsindex van de weefsels nog wat vergroot waardoor het meten van de cornea, kamerwater en lens mogelijk wordt. De integratiediepte van het weefsel kan worden ingesteld door de keuze van de diameter van het confocaal geplaatste fiber. Een fiber van $50 \mu \mathrm{m}$ bijvoorbeeld geeft een integratiediepte van $130 \mu \mathrm{m}$ in het weefsel. Hierdoor kunnen cornea, kamerwater en lens duidelijk van elkaar worden onderscheiden. Axiale scans met deze probe tonen de respectievelijke Raman spectra zoals in situ verkregen in konijnenogen.

In hoofdstuk 6 wordt getoond dat met deze nieuwe confocale probe een axiale hydratie gradiënt in de cornea kan worden gemeten. Hiervoor zijn in vivo experimenten bij het konijn verricht. Ook is de (de-)hydratie met succes als functie van de tijd gemeten. Hiervoor werd gebruik gemaakt van de water/proteine ofwel de $\mathrm{OH} / \mathrm{CH}$ verhouding, zowel onder fysiologische omstandigheden als bij gebruik van een dehydrerende topicaal toegediende zalf.

In hoofdstuk 7 is hydratie onderzoek onder fysiologische omstandigheden in lenzen van konijnenogen verricht. Dankzij verbetering in de methoden en technieken kon worden begonnen met het verminderen van de stralingsbelasting. De nog in hoofdstuk 5 gebruikte $25 \mathrm{~mW}$ gedurende $1 \mathrm{~s}$ werd hier gereduceerd tot $1 \mathrm{~mW}$ gedurende $0,1 \mathrm{~s}$. Hiermee is de techniek veilig geworden voor het oog.

In de laatste twee hoofdstukken 8 en 9 wordt het uiteindelijke doel van dit promotieonderzoek beschreven, namelijk de klinisch relevante toepassingen van de Raman techniek in patiënten. Hiertoe werd het vermogen van de laser gereduceerd tot $95 \mu \mathrm{W}$ bij een registratietijd van $1 \mathrm{~s}$. Door de grote openingshoek van de lichtbundel is de stralingsdichtheid op de retina laag genoeg om veilig te zijn.

In hoofdstuk 8 wordt siliconenolie in het oog aangetoond bij een patiènt met een troebele cornea. Bij het levende konijn was het mogelijk om in de voorste oogkamer kleine olie bellen aan te tonen.

En tenslotte wordt in hoofdstuk 9 aan de hand van patiënten onderzoek aangetoond dat het 
materiaal van geïmplanteerde lenzen betrouwbaar kan worden vastgesteld aan de hand van Raman spectra.

\section{Conclusies}

Teneinde de nauwkeurige en betrouwbare Raman spectroscopie om te bouwen tot een gereedschap om niet invasief en in vivo informatie over de oogweefsels, kamerwater en glasvocht te verkrijgen, is een confocaal optisch systeem ontwikkeld met een grote lichtsterkte en hoge optische kwaliteit. Dit element is bij het onderzoek een onmisbaar deel van de opstelling geworden.

Commercieel verkrijgbare systemen met een vergelijkbare functie zijn meestal veel lichtzwakker en missen de hier vereiste hoge optische nauwkeurigheid. Daarom is er een aanzienlijke inspanning verricht om de bestaande systemen te vervangen door een systeem dat uiteindelijk geschikt was voor gebruik in patiënten. De beperkte maximaal toegestane stralingsbelasting van de retina, vereiste dat de fotonen met Raman verschuiving met een hoge efficiency werden verzameld. Tevens was het noodzakelijk een optische dissectie techniek voor de ruimtelijke beheersing van het meten in de drie dimensionale ruimte in het oog toe te passen. De combinatie hoge verzamel efficiency van de fotonen met een optische dissectie techniek, leidde tot de confocale benadering. De validatie van de confocale opstelling bevestigde dat Raman spectroscopie geschikt is voor het verkrijgen van informatie over moleculaire eigenschappen van intraoculaire structuren in patiënten op een niet-invasieve wijze, hetgeen het doel was van dit proefschrift. Het klinisch gebruik van de nu ontwikkelde opstelling is beperkt, maar het is een eerste stap in de richting van verdere ontwikkeling van deze niet- invasieve techniek, niet alleen als diagnostisch hulpmiddel bij oogheelkundige pathologie, maar ook bij het onderzoeken van fysiologische processen in het oog.

\section{Toekomst}

De oogheelkundige Raman spectroscopische opstelling kan gezien worden als een keten waarin sommige schakels, zoals de lichtbron, slechts weinig kunnen worden verbeterd. Terwijl andere schakels, zoals de aard van de Raman spectroscopie, of de configuratie van de opstelling nader onderzoek rechtvaardigen. De spectrometer in dit onderzoek had een focale afstand van $0,5 \mathrm{~m}$ waarmee de vereiste resolutie werd verkregen. Vergroting van de spleet verlaagd de spectrale resolutie maar verhoogd de algemene gevoeligheid in de huidige opstelling. Wordt een fiber gebruikt die de ronde confocale spot omvormt tot een spleetvormig beeld, dan kan de hoge resolutie van de spectrometer behouden blijven. Het vermogen van de gebruikte argon laser $(5 \mathrm{~W})$ is veel hoger dan noodzakelijk. Dit hoog vermogen moest sterk worden gereduceerd om in het veilige gebied voor intraoculair gebruik te komen. Echter, andere Raman spectroscopische technieken dan de in dit onderzoek gebruikte techniek zouden de gevoeligheid en de nauwkeurigheid wel eens aanzienlijk kunnen verhogen. Het gebruik van twee golflengte excitatie is een interessante mogelijkheid omdat hiermee de bijdrage van fluorescentie aan het Raman signaal achteraf kan worden geminimaliseerd. Indien een specifieke stof een goed gedefinieerd Raman signaal geeft, kan reso- 
nantie Raman worden toegepast om de intensiteit van dat specifieke signaal te vergroten. De quantum efficiency van de huidige $C C D$ detectors in het zichtbaar gebied is niet ver van de fysische grens verwijderd. Ontwikkeling van detectoren met verhoogde quantum efficiency in het nabije infrarood kan van belang zijn hoewel de Raman intensiteit omgekeerd evenredig is met de vierde macht van de golflengte van het excitatielicht. Dus voor dit gebied zal dan meer laser vermogen en/of langere integratietijd noodzakelijk zijn. Voor het comfort van de patiënten is een integratietijd van seconden of korter aan te bevelen. Een voordeel van het gebruik van langere golflengten is, dat deze minder schadelijk zijn voor de retina. Er zal dus altijd een afweging moeten worden gemaakt tussen de gewenste golflengte van de excitatiebron, de gevoeligheid van de detector en de duur van de integratietijd.

De confocale opstelling werkt contactloos. Vanwege de vorm en de brekingsindex van het oog (overgang van $n=1$ naar $n=1.37$ bij de lucht - traanfilm scheiding) is het moeilijk om loodrecht op de optische as van het oog door het kamerwater te exciteren. De excitatiebundel zal richting retina worden afgebogen tenzij een extreme positie aan de zijkant van het oog wordt gehanteerd. Hiervoor zou een optisch element ontworpen kunnen worden dat de brekingsindex overgang lucht - traanfilm elimineert, bijvoorbeeld zoals een contactlens of contactglas. In de oogheelkunde is het gebruik hiervan zowel voor therapeutische als diagnostische doeleinden dagelijkse routine. Een op deze wijze gemodificeerd confocaal element dat in de buurt van $90^{\circ}$ t.o.v. de optische as van het oog werkt zou geschikt zijn om het kamerwater te onderzoeken, zonodig met hoger excitatievermogen, zonder de retina te beschadigen.

Er zijn verschillende stoffen in het kamerwater interessant om te onderzoeken: bijvoorbeeld phenylalanine is een belangrijk molecuul daar in de ziekte phenylketonurie, de verhoogde spiegel kan worden gedetecteerd waarmee een dieet kan worden ingesteld om de gewenste concentratie te verkrijgen. Andere interessante stoffen in het kamerwater zijn lactaat, ureum, glucose en aminozuren. Ook de farmacokinetiek, het transport en de bio-beschikbaarheid yan farmacologische stoffen kunnen dan worden bestudeerd.

Dieper in het oog is het glasvocht van groot diagnostisch belang daar dit waarschijnlijk een sleutelrol speelt in de pathofysiologie van retinale aandoeningen.

Een andere veelbelovende ontwikkeling in de signaal analyse van de Raman spectra is de Multivariabele Statistische Methode welke detectie van verschillende chemische substanties, die samen in het te onderzoeken substraat zijn opgelost, mogelijk maakt (bijvoorbeeld glucose, lactaat, ureum en aminozuren in kamerwater). Deze methode kan dus worden toegepast indien een aantal verscheidene parameters in de signalen aanwezig zijn, en een overlapping van verschillende Raman spectra moet worden ontrafeld of indien spectra zijn "begraven" onder interfererende structuren.

Gezien de vermelde mogelijkheden en de snelle ontwikkeling van nieuwe Raman technieken, kan het worden verwacht dat Raman spectroscopie in de nabije toekomst een significante rol kan spelen in het helpen van de oogarts om de verlangde informatie van verschillende oogstructuren te verkrijgen op een voor de patiënt minimaal belastende manier. 


\section{Dankwoord}

Een dankwoord schrijven is als afsluiting van dit proefschrift zeker geen makkelijke opgave, mijn lange termijn geheugen wordt zwaar beproefd...! Ik heb gekozen voor een chronologische volgorde daar dit ook het beste perspectief biedt om de stappen die genomen zijn te verduidelijken.

Allereerst wil ik dhr. J. Erckens en mevr. H. Erckens-Smeets bedanken zonder wiens hulp (1959), onvoorwaardelijke steun en geloof in mij, dit proefschrift nooit het levenslicht gezien zou hebben. Mama en papa bedankt!!

Dr. F. Petrij, beste Fred. Jouw fotografische kennis en enthousiasme (1985) hebben, mede dankzij fotoclub Contrast, een rol gespeeld bij het oplossen van optische vraagstukken.

Dr. F. Jongsma kwam al vroeg in mijn academische leven (1986) toen ik op zoek was naar een laser om hologrammen te kunnen maken. Beste Frans, sinds die tijd is jouw scherpe geest en inzicht in de optica een steun gebleven. Met genoegen denk ik terug aan het bouwen van de Michelson inteferometer of aan de Schlieren fotografie. Daarnaast heb je me geintroduceerd bij prof. van Gemert (1989) van het lasercentrum in het AMC, en later bij prof. Hendrikse (1994) in het AZM. Bij de totstandkoming van dit proefschrift heb je een cruciale rol gespeelt toen bleek dat de commerciële lichtgeleiders niet voldeden. Ik wist toen dat jouw optische inzichten de oplossing konden bieden en gelukkig stemde je toe om te helpen. De trollen en het zachte brood waren wel een bestraffing voor je, maar gelukkig heb je de Texaanse tijd doorstaan en ben je zelfs meerdere malen teruggekeerd!

Prof. dr. ir. M. van Gemert, beste Martin. In 'jouw' lasercentrum heb ik me kunnen bekwamen in het gebruik van lasers. En het doet me genoegen dat ik heb kunnen participeren in een lasercursus in 1990 en de gepulste dye laser mocht demonstreren aan de cursisten. Jouw vermogen om mensen te inspireren is uniek. Jouw connectie met Galveston heeft me in contact gebracht met dr. Motamedi.

Dr. ir. H. Sterenborg, beste Dick. Samenwerken met jouw blijft een belevenis, varierend van een 'sluiproute' voor de gepulste laser (1990) naar de ok in het AMC, tot muizen bestuderen (1993) in een Texaanse bunker.

In 1991 I visited the eye clinic of Dr. Tennyson Luke in Perumbavoor in India. I wish to thank Dr. Tennyson and his family for their hospitality. This experience changed my life and always stayed with me, and also helped me with the research.

Dr. M. Motamedi, dear Massoud. Thanks to you I could visit Texas (1992) and start my 'Raman' career. I thank you for your insight that my interest were more with eyes than with prostates. You teached me the principles of responsible scientific behaviour and always critical questioning the research to perform.

Dr. J. Wicksted, dear Jim. As the Raman expert from Oklahoma (1992) you introduced me to the Raman technique and our discussions lead to the investigation of the eye with the Raman technique (As we discussed many times: resistance is futile...). In the following years you always were available for help and advice. Visiting you and Carol in Oklahoma will always remain a pleasant memory.

Dr. W. March, dear Wayne. As the head of the department of ophthalmology in Galveston Texas, your support and enthusiasm was of extreme value to the succes of the thesis. The fellowship and the unrestricted Grant from the Research to Prevent Blindness foundation, 
ensured the continuation of the project. You proved to be able to encourage me to make the almost impossible task come through.

I wish to thank the collegues in Galveston who participated in the research Drs. S. Trocme, B. Millstein, D. Gold, and H. Li. Dr. E. van Kuijk, beste Erik. Bedankt voor de gastvrijheid, bedankt ook Virginia en Charly.

Mijn promotor Prof. dr. F. Hendrikse. Gedurende een bezoek aan Nederland (1993) tijdens mijn Texaanse tijd, heb ik op advies van dr. Jongsma een afspraak gemaakt met u om te praten over mijn onderzoek en een eventuele opleidingsplaats tot oogarts. Tijdens het gesprek werd duidelijk dat het Raman onderzoek interesse gewekt had bij $\mathbf{u}$ en dat mijn opleidingsplaats (1995) geregeld kon worden. Uw steun en vertrouwen in een goede afloop, al vond $\mathrm{u}$ het het soms wel wat lang duren..., waren van onschatbare waarde voor het tot stand komen van dit proefschrift.

Mr. B. Bell, dear Brent, thank you for your help with the spectrometer, laser and equipment. Even in weekends you were there.

Mijn opvolgers in Texas dr. N. Bauer, drs. E. Smit, en drs. K. Hosseini. Beste Noël, Eefke en Kamran, in de jaren na mijn Texaanse avontuur heb ik jullie leren kennen en waarderen en hebben we ook een vruchtbare samenwerking gehad welke hopenlijk in de toekomst kan worden gecontinueerd.

Prof. dr. H. Struijker Boudier, prof. dr. L. Koole, en prof. dr. ir. J. Janssen dank ik hierbij voor hun inspanningen als leden van de interne beoordelingscommissie.

De dames van de secretariaten oogheelkunde in het AZM zou ik willen bedanken voor hun hulp bij het ter beschikking stellen van de printer, schaar, ander materiaal en adviezen.

De poli medewerkers van oogheelkunde in het AZM zou ik willen bedanken voor hun nimmer aflatende interesse voor het proefschrift, en dan met name wanneer het feest zou zijn... Mevr. P. Bisschoff, beste Peggy. Jouw hulp bij het vervaardigen van dit boekje was van onschatbare waarde evenals je motivatie om dit snel tot een einde te brengen.

Finally, as a last minute participant, I would like to thank Ms. R. Sandra. Dear Raluca, you inspired me and gave me courage in the last difficult moments towards the ending of my thesis. 


\section{Curriculum Vitae:}

Roel Johan Erckens werd 4-2-59 geboren te Meerssen.

Het Atheneum diploma werd in 1979 verkregen aan de Scholengemeenschap Stella Maris te Maastricht.

Vervolgens een jaar farmacie gestudeerd aan de Rijksuniversiteit te Leiden (1980-1981).

De geneeskunde studie aan de Rijksuniversiteit te Maastricht werd in 1982 gestart met in 1991 het basisarts getuigschrift.

Het wetenschappelijk onderzoek aan de University of Texas Medical Branch te Galveston TX U.S.A. werd begonnen in 1992 en eindigde in 1995.

Specialisatie tot oogarts bij prof. dr. F. Hendrikse in het Academisch Ziekenhuis te Maastricht, begonnen 1-6-95 en voltooid 1-7-00.

Auteur is momenteel werkzaam als oogarts in het Academisch Ziekenhuis te Maastricht. 


\section{Publications}

Papers:

- Sterenborg H, de Rijeke Th, Wiersma J, Erckens R, Jongsma F. High-speed photographic evaluation of endoscopic lithotripsy devices. Urol Res 1991;19:381-385.

- Wicksted JP, Erckens RJ, Motamedi M, March WF. Raman spectroscopy studies of metabolic concentrations in aqueous solutions and aqueous humor specimens. Applied Spectroscopy 1995;49:987-993.

- Goetz M, Cote G, Erckens R, March W, Motamedi M. Application of a multivariate technique to Raman spectra for quantification of body chemicals. IEEE Trans Biomed Eng 1995;42:728-731.

- Erckens RJ, Wicksted JP, March WF, Motamedi M. Raman spectroscopy for noninvasive characterization of ocular tissue: Potential for detection of biological molecules. Journal of Raman Spectroscopy 1997;28:293-299.

- Jongsma F, Erckens R, Wicksted J, Bauer N, Hendrikse F, March W, Motamedi M. Confocal Raman spectroscopy system for noncontact scanning of ocular tissues: An in vitro study. Optical Engineering 1997;36:3193-3199.

- Smit E, Erckens R, Hendrikse F, Motamedi M, Wicksted J, March W. Identification of intraocular lens materials using confocal Raman spectroscopy. J Cataract refract Surg 1999;25:1498-1504.

- Wicksted J, Bauer N, Erckens R, Jongsma F, Smit E, Motamedi M, March W. Noninvasive assessment of ocular tissue using confocal Raman spectroscopy. Proceedings of the SPIE conference on Biomedical Applications of Raman Spectroscopy; volume 3608, 1999 San Jose, California.

- Erckens RJ, March WF, Jongsma FH Wicksted JP, Hendrikse F, Smit E, Motamedi M. Noninvasive Raman spectroscopic identification of the intraocular lens material in the living human eye. J Cataract refract Surg, In press.

- Erckens, Jongsma, Wicksted, Hendrikse, March, Motamedi. Raman spectroscopy in ophthalmology: From experimental tool to applications in vivo. [Review] Lasers Med Sci. In press.

- Erckens, Jongsma, Wicksted, Hendrikse, March, Motamedi. Drug induced corneal hydration changes monitored in vivo by noninvasive confocal Raman spectroscopy. J Raman Spectr, In press.

\section{Abstracts}

- "5 th International Congress of the European Laser Association 1990 te Graz (AU): Endoscopic Lithotripsy: an ultra high speed photographic evaluation.

- JERMOV Montpellier 1995: Monitoring the water gradient in the in vivo rabbit cornea using confocal Raman spectroscopy.

- NOG 1995: Raman spectroscopie in konijneogen, een in vivo experiment.

- NOG 1997: In vivo Raman spectroscopie van de ooglens van het konijn.

- ARVO Ft. Lauderdale 1996: Noninvasive in vivo assesment of comeal dehydration in the rabbit using confocal Raman spectroscopy. 
- NOG 1998: Het detecteren van subconjunctivaal ingespoten antibiotica met behulp van Raman spectroscopie bij NZW konijnen in vivo.

- NOG 1999: Discrepantie in progressie van diabetische retinopathie na cataract extractie ten nadele van het pseudofake oog (klinische casuistiek).

- ARVO Ft. Lauderdale 2000: Noninvasive determination of intraocular lenses in vivo using confocal Raman spectroscopy.

- NOG 2001: Spectroscopische detectie van de soort geïmplanteerde IOL bij patiënten. 